\title{
LINEAR INVISCID DAMPING FOR A CLASS OF MONOTONE SHEAR FLOW IN SOBOLEV SPACES
}

\author{
DONGYI WEI, ZHIFEI ZHANG, AND WEIREN ZHAO
}

\begin{abstract}
In this paper, we prove the decay estimates of the velocity and $H^{1}$ scattering for the 2D linearized Euler equations around a class of monotone shear flow in a finite channel. Our result is consistent with the decay rate predicted by Case in 1960.
\end{abstract}

\section{INTRODUCTION}

In this paper, we consider the $2 \mathrm{D}$ incompressible Euler equations in a finite channel $\{(x, y)$ : $x \in \mathbb{T}, y \in[0,1]\}:$

$$
\left\{\begin{array}{l}
\partial_{t} V+V \cdot \nabla V+\nabla P=0, \\
\nabla \cdot V=0 \\
V^{2}(t, x, 0)=V^{2}(t, x, 1)=0 \\
\left.V\right|_{t=0}=V_{0}(x, y) .
\end{array}\right.
$$

where $V=\left(V^{1}, V^{2}\right)$ and $P$ denote the velocity and the pressure of the fluid respectively. Let $\omega=\partial_{x} V^{2}-\partial_{y} V^{1}$ be the vorticity, which satisfies

$$
\omega_{t}+V \cdot \nabla \omega=0 .
$$

It is well-known that the 2D incompressible Euler equations are globally well-posed for smooth data $[6,17]$. However, the long time behaviour of the solution is widely open. We refer to $[11,8]$ for recent relevance results.

We are concerned with the asymptotic stability of the 2D linearized Euler equations around the shear flow $(u(y), 0)$, which is a steady solution of 2D Euler equations. The linearized Euler equations around a shear flow $(u(y), 0)$ take

$$
\left\{\begin{array}{l}
\partial_{t} \omega+\mathcal{L} \omega=0 \\
\left.\omega\right|_{t=0}=\omega_{0}(x, y)
\end{array}\right.
$$

where $\mathcal{L}=u(y) \partial_{x}+u^{\prime \prime}(y) \partial_{x}(-\Delta)^{-1}$.

The stability of 2-D Euler equations is a very active field in Physics and Mathematics [9], especially for shear flows [22]. Rayleigh's inflection point theorem gives a necessary condition for linear stability of shear flow: $u(y)$ has no infection points [20]. Arnold's theorem gives a sufficient condition for nonlinear Liapunov stability of shear flow [1]:

$$
0<c_{1} \leq \frac{u(y)}{u^{\prime \prime}(y)} \leq c_{2}<+\infty .
$$

Lin [13] provided a large classes of unstable shear flows. We refer to [2, 14, 10, 24] and references therein for nonlinear instability.

Date: September 29, 2015. 
In 1907, Orr [19] observed that the velocity tends to zero as $t \rightarrow+\infty$ for the linearized Euler equations around Couette flow $(y, 0)$. In this case, the linearized vorticity equation becomes

$$
\omega_{t}+y \partial_{x} \omega=0
$$

Thus, $\omega(t, x, y)=\omega_{0}(x-t y, y)$. Especially, in the case of the infinite channel $\mathbb{T} \times \mathbf{R}$, the velocity can be explicitly solved. Indeed, let $\psi$ be the stream function, i.e., $\left(V^{1}, V^{2}\right)=$ $\left(\partial_{y} \psi,-\partial_{x} \psi\right)$. Hence,

$$
-\Delta \psi=\omega .
$$

Since we deduce by taking Fourier transform to (1.4) that

$$
\widehat{\psi}(t, \alpha, \xi)=\frac{\widehat{\omega}_{0}(\alpha, \xi+\alpha t)}{\alpha^{2}+|\xi|^{2}}
$$

which implies that $V^{1}$ decays at $t^{-1}$, while $V^{2}$ decays at $t^{-2}$. In the case of finite channel, Case [7] gave a formal proof of $t^{-1}$ decay for the velocity. Recently, Lin and Zeng [16] present the optimal linear decay estimates of the velocity for the data in Sobolev space. More precisely, if $\int_{\mathbb{T}} \omega_{0}(x, y) d x=0$, then it holds that

1. if $\omega_{0}(x, y) \in H_{x}^{-1} H_{y}^{1}$, then

$$
\|V(t)\|_{L^{2}}=O\left(\frac{1}{t}\right)
$$

2. if $\omega_{0}(x, y) \in H_{x}^{-1} H_{y}^{2}$, then

$$
\left\|V^{2}(t)\right\|_{L^{2}}=O\left(\frac{1}{t^{2}}\right)
$$

Such inviscid damping is surprising for a time reversible system. The basic mechanism leading to this phenomena is vorticity mixing driven by shear flow, which may be related to the appearance of coherent structures in $2 \mathrm{D}$ turbulence. This behaviour is similar to Landau damping [12], which predicted the rapid decay of the electric field of the linearized Vlasov equation around homogeneous equilibrium.

It is a very difficult problem to extend linear damping to nonlinear damping. Mouhot and Villani [18] made a breakthrough and proved nonlinear Landau damping for the perturbation in Gevrey class(see also [4]). Motivated by [18], Bedrossian and Masmoudi also proved the nonlinear inviscid damping of 2D Euler equations around Couette flow in infinite channel still for the perturbation in Gevrey class. Lin and Zeng [16, 15] also show that nonlinear damping is not true for the perturbation in low regularity Sobolev spaces.

The goal of this paper is to prove linear damping for the 2D Euler equations around general shear flow. In this case, there are few rigorous mathematical results. Case [7] gave the formal prediction for the decay of the velocity by using the Laplace transform and the leading singularity of the resolvent. Rosencrans and Sattinger [21] gave $t^{-1}$ decay of the stream function with a continuous spectrum projection for analytic monotone shear flow. Stepin [23] proved $t^{-\nu}\left(\nu<\mu_{0}\right)$ decay of the stream function for the monotone shear flow $u(y) \in C^{2+\mu_{0}}\left(\mu_{0}>\frac{1}{2}\right)$ without inflection point. In a very interesting paper [5], Bouchet and Morita predicted similar decay estimates of the velocity for a class of stable shear flow with stationary streamlines by using the Laplace transform and an important observation: depletion phenomena of the vorticity at the stationary streamlines. More precisely, they formally proved that

$$
\omega(t, x, y) \sim \omega_{\infty}(x, y) \exp (-i k u(y) t)+O\left(t^{-\gamma}\right) \quad \text { as } t \rightarrow+\infty,
$$


where $\omega_{\infty}\left(x, y_{c}\right)=0$ at stationary points $y_{c}$ of $u(y)$.

In a recent paper, C. Zillinger [25] proved the same decay estimates as those of Couette flow given by Lin and Zeng [16] for a class of monotone shear flow in Sobolev spaces. However, his result imposed a strong assumption that $L\left\|u^{\prime \prime}\right\|_{W^{3, \infty}}$ is small, where $L$ is the wave-length with respect to $x$. Moreover, he also required that the initial vorticity vanishes on $y=0,1$ in the case of finite channel. Thus, the linear inviscid damping is still open for general monotone shear flow.

The main result of this paper is stated as follows.

Theorem 1.1. Let $u(y) \in C^{4}([0,1])$ be a monotone function. Suppose that the linearized operator $\mathcal{L}$ has no embedding eigenvalues. Assume that $\int_{\mathbb{T}} \omega_{0}(x, y) d x=0$ and $P_{\mathcal{L}} \omega_{0}=0$, where $P_{\mathcal{L}}$ is the spectral projection to $\sigma_{d}(\mathcal{L})$. Then it holds that

1. if $\omega_{0}(x, y) \in H_{x}^{-1} H_{y}^{1}$, then

$$
\|V(t)\|_{L^{2}} \leq \frac{C}{\langle t\rangle}\left\|\omega_{0}\right\|_{H_{x}^{-1} H_{y}^{1}}
$$

2. if $\omega_{0}(x, y) \in H_{x}^{-1} H_{y}^{2}$, then

$$
\left\|V^{2}(t)\right\|_{L^{2}} \leq \frac{C}{\langle t\rangle^{2}}\left\|\omega_{0}\right\|_{H_{x}^{-1} H_{y}^{2}}
$$

3. if $\omega_{0}(x, y) \in H_{x}^{-1} H_{y}^{k}$ for $k=0,1$, there exists $\omega_{\infty}(x, y) \in H_{x}^{-1} H_{y}^{k}$ such that

$$
\left\|\omega(t, x+t u(y), y)-\omega_{\infty}\right\|_{L^{2}} \longrightarrow 0 \quad \text { as } t \rightarrow+\infty .
$$

Let us give some remarks on Theorem 1.1.

- If $u(y)$ has no inflection points, then $\mathcal{L}$ has no eigenvalues. In Remark 6.4, we will present a sufficient condition on $u(y)$ in the case when $u(y)$ has inflection points so that $\mathcal{L}$ has no eigenvalues.

- If the wave-length $L$ with respect to $x$ is suitably small, then $\mathcal{L}$ has no embedding eigenvalues. In Lemma 6.1, we will present a sufficient and necessary condition on $u(y)$ so that $\mathcal{R}_{\alpha}$ has no embedding eigenvalues.

- By Zillinger's recent result [26], the $L_{x}^{2} H_{y}^{2}$ norm of $W(t, x, y) \triangleq \omega(t, x+t u(y), y)$ may blow up. Thus, it is in general unexpected for the $H^{2}$ scattering.

- Our proof strongly relies on the monotonicity of $u(y)$. Thus, it remains unknown whether the decay estimates predicted by Bouchet and Morita [5] for stable shear flow with stationary streamlines can be justified.

- Nonlinear inviscid damping is a challenging question even for the analytic perturbation.

\section{Sketch of the PROOF}

Our proof is based on the representation formula of the solution

$$
\widehat{\psi}(t, \alpha, y)=\frac{1}{2 \pi i} \int_{\partial \Omega} e^{-i \alpha t c}\left(c-\mathcal{R}_{\alpha}\right)^{-1} \widehat{\psi}(0, \alpha, y) d c,
$$

where $\mathcal{R}_{\alpha}$ is the Rayleigh operator defined by (3.3). Under the assumption that $\mathcal{L}$ has no embedding eigenvalues and $P_{\mathcal{L}} \omega_{0}=0$, the asymptotic behaviour of the solution is only related to the continuous spectrum. Thus, we only need to study

$$
\widehat{\psi}(t, \alpha, y)=\frac{1}{2 \pi i} \int_{u(0)}^{u(1)} e^{-i \alpha t c} \lim _{\epsilon \rightarrow 0+}\left(\left(c-i \epsilon-\mathcal{R}_{\alpha}\right)^{-1}-\left(c+i \epsilon-\mathcal{R}_{\alpha}\right)^{-1}\right) \widehat{\psi}(0, \alpha, y) d c .
$$


To establish the estimates of the resolvent $\left(c-\mathcal{R}_{\alpha}\right)^{-1}$, we need to study the inhomogeneous Rayleigh equation

$$
\left\{\begin{array}{l}
\Phi^{\prime \prime}-\alpha^{2} \Phi-\frac{u^{\prime \prime}}{u-c} \Phi=f \\
\Phi(0)=\Phi(1)=0
\end{array}\right.
$$

with $f=\frac{\widehat{\omega}_{0}(\alpha, y)}{i \alpha(u-c)}$. Indeed, it holds that

$$
\begin{aligned}
& \lim _{\epsilon \rightarrow 0+}\left(\left(c-i \epsilon-\mathcal{R}_{\alpha}\right)^{-1}-\left(c+i \epsilon-\mathcal{R}_{\alpha}\right)^{-1}\right) \widehat{\psi}(0, \alpha, y) \\
& \quad=i \alpha \lim _{\epsilon \rightarrow 0+}(\Phi(y, c+i \epsilon)-\Phi(y, c-i \epsilon)) \triangleq i \alpha \widetilde{\Phi}(y, c) .
\end{aligned}
$$

Thus, we obtain

$$
\widehat{\psi}(t, \alpha, y)=\frac{1}{2 \pi} \int_{u(0)}^{u(1)} e^{-i \alpha t c} \alpha \widetilde{\Phi}(y, c) d c .
$$

Formally, if one can show that $\widetilde{\Phi}(y, c) \in W^{2,1}$ in $c$, then integration by parts gives

$$
\widehat{\psi}(t, \alpha, y) \sim O\left(t^{-2}\right) \int_{u(0)}^{u(1)} e^{-i \alpha t c} \partial_{c}^{2} \widetilde{\Phi}(y, c) d c \sim O\left(t^{-2}\right) .
$$

One of main difficulties is that the solution $\Phi(y, c)$ of the inhomogeneous Rayleigh equation has a singularity of order $\left(y-y_{c}\right) \log \left|y-y_{c}\right|$ with $y_{c}=u^{-1}(c)$ (see $\left.[7,5]\right)$. Thus, $\partial_{c}^{2} \widetilde{\Phi}(y, c) \sim$ $\frac{1}{y-y_{c}} \notin L^{1}$. This may be the main reason why the authors in $[7,21,23]$ only obtained the $O\left(t^{-1}\right)$ decay of the stream function even in the analytic framework.

Indeed, in Section 6 and 7 , we will show that $\alpha \widetilde{\Phi}(y, c)=2 \rho(c) \mu(c) \Gamma(y, c)$ with $\rho(c)=$ $(c-u(0))(u(1)-c)$ and

$$
\Gamma(y, c)= \begin{cases}\phi(y, c) \int_{0}^{y} \frac{1}{\phi(z, c)^{2}} d z & 0 \leq y<y_{c} \\ \phi(y, c) \int_{1}^{y} \frac{1}{\phi(z, c)^{2}} d z & y_{c}<y \leq 1\end{cases}
$$

where $\phi(y, c)$ is the solution of the homogeneous Rayleigh equation:

$$
\left\{\begin{array}{l}
\phi^{\prime \prime}-\alpha^{2} \phi-\frac{u^{\prime \prime}}{u-c} \phi=0 \\
\phi\left(y_{c}, c\right)=0, \quad \phi^{\prime}\left(y_{c}, c\right)=u^{\prime}\left(y_{c}\right)
\end{array}\right.
$$

Thus, $\phi(y, c)$ has the behaviour near $y_{c}$ :

$$
\phi(y, c) \sim u^{\prime}\left(y_{c}\right)\left(y-y_{c}\right)+\frac{u^{\prime \prime}\left(y_{c}\right)}{2 u^{\prime}\left(y_{c}\right)}\left(y-y_{c}\right)^{2}+o\left(\left(y-y_{c}\right)^{2}\right),
$$

which implies that $\Gamma(y, c)$ has the behaviour near $y_{c}$ :

$$
\Gamma(y, c) \sim a+b\left(y-y_{c}\right) \log \left|y-y_{c}\right|,
$$

for some constants $a, b$.

The goal of Section 4-Section 5 is to obtain various kinds of uniform estimates for the solution of the homogeneous Rayleigh equations. The assumption that $u(y)$ is monotone plays an important role. In Section 8, we will establish the weighted $H^{2}$ estimate of $\mu(c)$, where we need to assume that $\mathcal{L}$ has no embedding eigenvalues.

Based on the solution formula (2.1), using the weighted $H^{2}$ estimate of $\mu(c)$ and $L^{p}$ boundedness for various kinds of singular integral operators, we will establish the $H_{y}^{2}$ estimate of 
$W(t, x, y)=\omega(t, x+t u(y), y)$ in Section 9. In fact, we only prove the weighted $H_{y}^{2}$ estimate, while $H_{y}^{2}$ bound is impossible in general.

With the uniform Sobolev estimates of the vorticity, the decay estimates can be deduced by following the dual argument introduced by Lin and Zeng [16].

In the appendix, we will establish $L^{p}$ boundedness for various kinds of singular integral operators, which was used in Section 8 and Section 9.

\section{Spectrum of the Linearized operator}

In terms of the stream function $\psi$, the linearized Euler equations take

$$
\partial_{t} \Delta \psi+u(y) \partial_{x} \Delta \psi-u^{\prime \prime}(y) \partial_{x} \psi=0 .
$$

Taking the Fourier transform in $x$, we get

$$
\left(\partial_{y}^{2}-\alpha^{2}\right) \partial_{t} \widehat{\psi}=i \alpha\left(u^{\prime \prime}(y)-u(y)\left(\partial_{y}^{2}-\alpha^{2}\right)\right) \widehat{\psi} .
$$

Inverting the operator $\left(\partial_{y}^{2}-\alpha^{2}\right)$, we find

$$
-\frac{1}{i \alpha} \partial_{t} \widehat{\psi}=\mathcal{R}_{\alpha} \widehat{\psi}
$$

where

$$
\mathcal{R}_{\alpha} \widehat{\psi}=-\left(\partial_{y}^{2}-\alpha^{2}\right)^{-1}\left(u^{\prime \prime}(y)-u\left(\partial_{y}^{2}-\alpha^{2}\right)\right) \widehat{\psi} .
$$

It is easy to show that

$$
\bigcup_{\alpha} \sigma_{d}\left(i \alpha \mathcal{R}_{\alpha}\right)=\sigma_{d}(\mathcal{L})
$$

Let us recall some classical results for the spectrum $\sigma\left(\mathcal{R}_{\alpha}\right)$ of the operator $\mathcal{R}_{\alpha}$ (see [21, 23]for more details).

1. The spectrum $\sigma\left(\mathcal{R}_{\alpha}\right)$ is compact;

2. The continuous spectrum $\sigma_{c}\left(\mathcal{R}_{\alpha}\right)$ is contained in the range $\operatorname{Ran}(u)$ of $u(y)$;

3. The eigenvalues of $\mathcal{R}_{\alpha}$ can not cluster except possibly along on $\operatorname{Ran}(u)$;

4. If $u(y)$ has no infection points in $[0,1]$, then $\mathcal{R}_{\alpha}$ has no embedding eigenvalues;

5. If $u(y)$ has inflection points, then $\mathcal{R}_{\alpha}$ has no embedding eigenvalues for $\alpha^{2}>\alpha_{\text {max }}^{2}$, where

$$
\alpha_{\text {max }}^{2} \stackrel{\text { def }}{=}-\inf _{y_{c}: u^{\prime \prime}\left(y_{c}\right)=0} \inf _{\phi \in H_{0}^{1}(0,1)} \frac{\int_{0}^{1}\left|\phi^{\prime}(y)\right|^{2}-\frac{u^{\prime \prime}(y)}{u(y)-u\left(y_{c}\right)}|\phi(y)|^{2} d y}{\int_{0}^{1}|\phi(y)|^{2} d y} .
$$

Proof of 4. Let $\phi \in H^{2}(0,1) \cap H_{0}^{1}(0,1)$ be an eigenfunction of $\mathcal{R}_{\alpha}$ with the eigenvalue $c \in \operatorname{Ran}(u)$, i.e., $\mathcal{R}_{\alpha} \phi=c \phi$, which can be reduced to the well-known Rayleigh equation:

$$
(u-c)\left(\phi^{\prime \prime}-\alpha^{2} \phi\right)-u^{\prime \prime} \phi=0 \text {. }
$$

If $u^{\prime \prime}\left(u^{-1}(c)\right) \neq 0, \phi\left(u^{-1}(c)\right)=0$ by (3.4). Taking the inner product with $\frac{\phi}{u-c}$ on both sides of $(3.4)$, we obtain

$$
\int_{0}^{1} \phi^{\prime \prime} \phi d y-\alpha^{2} \int_{0}^{1}|\phi|^{2} d y-\int_{0}^{1} u^{\prime \prime} \frac{|\phi|^{2}}{u-c} d y=0 .
$$

Integration by parts gives

$$
-\int_{0}^{1}\left|\phi^{\prime}-u^{\prime} \frac{\phi}{u-c}\right|^{2} d y-\alpha^{2} \int_{0}^{1}|\phi|^{2} d z=0
$$


which implies that $\phi \equiv 0$. Thus, $u^{\prime \prime}\left(u^{-1}(c)\right)=0$ if $c$ is an embedding eigenvalue.

Proof of 5. If $c$ is an embedding eigenvalue with eigenfunction $\phi$, then we have

$$
\int_{0}^{1}\left|\phi^{\prime}\right|^{2}-\frac{u^{\prime \prime}}{u-c}|\phi|^{2} d y+\alpha^{2} \int_{0}^{1}|\phi|^{2} d y=0 .
$$

However, for $\alpha^{2}>\alpha_{\max }^{2}$, we have

$$
\int_{0}^{1}\left|\phi^{\prime}\right|^{2}-\frac{u^{\prime \prime}}{u-c}|\phi|^{2} d y+\alpha^{2} \int_{0}^{1}|\phi|^{2} d y \neq 0 .
$$

In Lemma 6.1, we will present a sufficient and necessary condition on $u(y)$ so that $\mathcal{R}_{\alpha}$ has no embedding eigenvalues. In Remark 6.4, we will present a sufficient condition so that $\mathcal{R}_{\alpha}$ has no eigenvalues, which in particular implies that $\mathcal{R}_{\alpha}$ has no eigenvalues if $u(y)$ has inflection points in $[0,1]$ and $\alpha^{2}>\alpha_{\text {max }}^{2}$.

Let $\Omega$ be a simple connected domain including the spectrum $\sigma\left(\mathcal{R}_{\alpha}\right)$ of $\mathcal{R}_{\alpha}$. We have the following representation formula of the solution to (3.2):

$$
\widehat{\psi}(t, \alpha, y)=\frac{1}{2 \pi i} \int_{\partial \Omega} e^{-i \alpha t c}\left(c-\mathcal{R}_{\alpha}\right)^{-1} \widehat{\psi}(0, \alpha, y) d c .
$$

Thus, the large time behaviour of the solution $\widehat{\psi}(t, \alpha, y)$ is reduced to the study of the resolvent $\left(c-\mathcal{R}_{\alpha}\right)^{-1}$.

\section{The homogeneous Rayleigh equation}

To study the resolvent $\left(c-\mathcal{R}_{\alpha}\right)^{-1}$, we first construct a smooth solution of the homogeneous Rayleigh equation on $[0,1]$ :

$$
(u-c)\left(\phi^{\prime \prime}-\alpha^{2} \phi\right)-u^{\prime \prime} \phi=0,
$$

where the complex constant $c$ will be taken in four kinds of domains defined by

$$
\begin{aligned}
& D_{0} \triangleq\{c \in[u(0), u(1)]\}, \\
& D_{\epsilon_{0}} \triangleq\left\{c=c_{r}+i \epsilon, c_{r} \in[u(0), u(1)], 0<|\epsilon|<\epsilon_{0}\right\}, \\
& B_{\epsilon_{0}}^{l} \triangleq\left\{c=u(0)+\epsilon e^{i \theta}, 0<\epsilon<\epsilon_{0}, \frac{\pi}{2} \leq \theta \leq \frac{3 \pi}{2}\right\}, \\
& B_{\epsilon_{0}}^{r} \triangleq\left\{c=u(1)-\epsilon e^{i \theta}, 0<\epsilon<\epsilon_{0}, \frac{\pi}{2} \leq \theta \leq \frac{3 \pi}{2}\right\},
\end{aligned}
$$

for some $\epsilon_{0} \in(0,1)$. We denote

$$
\Omega_{\epsilon_{0}} \triangleq D_{0} \cup D_{\epsilon_{0}} \cup B_{\epsilon_{0}}^{l} \cup B_{\epsilon_{0}}^{r} .
$$

In the sequel, we always assume that $u(y) \in C^{4}([0,1])$ and satisfies $u^{\prime}(y) \geq c_{0}$ for some $c_{0}>0$.

4.1. Functional space and the integral operator. Given $|\alpha| \geq 1$, let $A$ be a constant larger than $C|\alpha|$ with $C \geq 1$ only depending on $c_{0}$ and $\|u\|_{C^{4}}$.

Definition 4.1. For a function $f(y, c)$ defined on $[0,1] \times \Omega_{\epsilon_{0}}$, we define

$$
\begin{aligned}
& \|f\|_{X_{0}} \stackrel{\text { def }}{=} \sup _{(y, c) \in[0,1] \times D_{0}}\left|\frac{f(y, c)}{\cosh \left(A\left(y-y_{c}\right)\right)}\right|, \\
& \|f\|_{X} \stackrel{\text { def }}{=} \sup _{(y, c) \in[0,1] \times D_{\epsilon_{0}}}\left|\frac{f(y, c)}{\cosh \left(A\left(y-y_{c}\right)\right)}\right|,
\end{aligned}
$$


LINEAR INVISCID DAMPING FOR A CLASS OF MONOTONE SHEAR FLOW IN SOBOLEV SPACES 7

$$
\begin{aligned}
& \|f\|_{X_{l}} \stackrel{\text { def }}{=} \sup _{(y, c) \in[0,1] \times B_{\epsilon_{0}}^{l}}\left|\frac{f(y, c)}{\cosh (A y)}\right|, \\
& \|f\|_{X_{r}} \stackrel{\text { def }}{=} \sup _{(y, c) \in[0,1] \times B_{\epsilon_{0}}^{r}}\left|\frac{f(y, c)}{\cosh (A(y-1))}\right|,
\end{aligned}
$$

where $y_{c}=u^{-1}\left(c_{r}\right)$ with

$$
c_{r}=\operatorname{Rec} \quad \text { for } c \in D_{\epsilon_{0}}, \quad c_{r}=u(0) \quad \text { for } c \in B_{\epsilon_{0}}^{l}, \quad c_{r}=u(1) \quad \text { for } c \in B_{\epsilon_{0}}^{r} .
$$

Definition 4.2. For a function $f(y, c)$ defined on $[0,1] \times \Omega_{\epsilon_{0}}$, we define

$$
\begin{aligned}
& \|f\|_{Y_{C}} \stackrel{\text { def }}{=} \sum_{k=0}^{2} \sum_{|\beta|=k} A^{-k}\left\|\partial^{\beta} f\right\|_{X_{0}}, \\
& \|f\|_{Y} \stackrel{\text { def }}{=}\|f\|_{X}+\frac{1}{A}\left(\left\|\partial_{x} f\right\|_{X}+\left\|\partial_{c} f\right\|_{X}\right), \\
& \|f\|_{Y_{l}} \stackrel{\text { def }}{=}\|f\|_{X_{l}}+\frac{1}{A}\left(\left\|\partial_{x} f\right\|_{X_{l}}+\left\|\partial_{c} f\right\|_{X_{l}}\right), \\
& \|f\|_{Y_{r}} \stackrel{\text { def }}{=}\|f\|_{X_{r}}+\frac{1}{A}\left(\left\|\partial_{x} f\right\|_{X_{r}}+\left\|\partial_{c} f\right\|_{X_{r}}\right) .
\end{aligned}
$$

Definition 4.3. Let $y_{c}=u^{-1}\left(c_{r}\right)$ with $c_{r}$ defined by (4.3). The integral operator $T$ is defined by

$$
T \triangleq T_{0} \circ T_{2,2} \stackrel{\operatorname{def}}{=} \int_{y_{c}}^{y} \frac{1}{\left(u\left(y^{\prime}\right)-c\right)^{2}} \int_{y_{c}}^{y^{\prime}} f(z, c)(u(z)-c)^{2} d z d y^{\prime}
$$

where

$$
\begin{aligned}
& T_{0} f(y, c) \stackrel{\text { def }}{=} \int_{y_{c}}^{y} f(z, c) d z, \\
& T_{k, j} f(y, c) \stackrel{\text { def }}{=} \frac{1}{(u(y)-c)^{j}} \int_{y_{c}}^{y} f(z, c)(u(z)-c)^{k} d z, \quad j \leq k+1 .
\end{aligned}
$$

Lemma 4.4. There exists a constant $C$ independent of $A$ such that

$$
\begin{aligned}
& \|T f\|_{Y_{C}} \leq \frac{C}{A^{2}}\|f\|_{Y_{C}}, \quad\|T f\|_{Y} \leq \frac{C}{A^{2}}\|f\|_{Y}, \\
& \|T f\|_{Y_{l}} \leq \frac{C}{A^{2}}\|f\|_{Y_{l}}, \quad\|T f\|_{Y_{r}} \leq \frac{C}{A^{2}}\|f\|_{Y_{r}} .
\end{aligned}
$$

Proof. We only prove the first inequality. The proof of the other inequalities is similar. A direct calculation shows

$$
\begin{aligned}
\left\|T_{0} f\right\|_{X_{0}} & =\sup _{(y, c) \in[0,1] \times D_{0}}\left|\frac{1}{\cosh A\left(y-y_{c}\right)} \int_{y_{c}}^{y} \frac{f(z, c)}{\cosh A\left(z-y_{c}\right)} \cosh A\left(z-y_{c}\right) d z\right| \\
& \leq \sup _{(y, c) \in[0,1] \times D_{0}}\left|\frac{1}{\cosh A\left(y-y_{c}\right)} \int_{y_{c}}^{y} \cosh A\left(z-y_{c}\right) d z\right|\|f\|_{X_{0}} \\
& \leq \frac{1}{A}\|f\|_{X_{0}},
\end{aligned}
$$

and

$$
\left\|T_{2,2} f\right\|_{X_{0}} \leq \sup _{(y, c) \in[0,1] \times D_{0}}\left|\frac{y-y_{c}}{\cosh A\left(y-y_{c}\right)} \int_{0}^{1} \cosh t A\left(y-y_{c}\right) d t\right|\|f\|_{X_{0}}
$$


which imply

$$
\|T f\|_{X_{0}} \leq \frac{C}{A^{2}}\|f\|_{X_{0}}
$$

It is easy to see that

$$
\begin{aligned}
& \partial_{y} T f(y, c)=T_{2,2} f(y, c), \\
& \partial_{c} T f(y, c)=2 T_{0} \circ T_{2,3} f(y, c)-2 T_{0} \circ T_{1,2} f(y, c)+T \partial_{c} f(y, c),
\end{aligned}
$$

and

$$
\begin{aligned}
\left\|T_{k, k+1} f\right\|_{X_{0}} & \leq C \sup _{(y, c) \in[0,1] \times D_{0}}\left|\frac{1}{\cosh A\left(y-y_{c}\right)} \int_{0}^{1} \cosh t A\left(y-y_{c}\right) d t\right|\|f\|_{X_{0}} \\
& \leq C\|f\|_{X_{0}},
\end{aligned}
$$

which along with (4.4) yield

$$
\left\|\partial_{y, c} T f\right\|_{X_{0}} \leq \frac{C}{A}\|f\|_{X_{0}}+\frac{C}{A^{2}}\left\|\partial_{c} f\right\|_{X_{0}} .
$$

Using the formula

$$
T_{k, k+1} f(y, c)=\int_{0}^{1} \frac{\left(\int_{0}^{1} u^{\prime}\left(y_{c}+s t\left(y-y_{c}\right)\right) d s\right)^{k}}{\left(\int_{0}^{1} u^{\prime}\left(y_{c}+s\left(y-y_{c}\right)\right) d s\right)^{k+1}} f\left(y_{c}+t\left(y-y_{c}\right), c\right) t^{k} d t,
$$

we can deduce that

$$
\left\|\partial_{y, c}^{2} T_{k, k+1} f\right\|_{X_{0}} \leq C \sum_{|\beta| \leq 2}\left\|\partial^{\beta} f\right\|_{X_{0}} .
$$

Then by a similar argument leading to (4.5), we obtain

$$
\left\|\partial_{y, c}^{2} T f\right\|_{X_{0}} \leq C\|f\|_{X_{0}}+\frac{C}{A}\left\|\partial_{y, c} f\right\|_{X_{0}}+\frac{C}{A^{2}}\left\|\partial_{y, c}^{2} f\right\|_{X_{0}} .
$$

Putting (4.4)-(4.6) together, we conclude the first inequality.

\subsection{Existence of the solution.}

Proposition 4.5. There exists a solution $\phi(y, c) \in C^{1}\left([0,1] \times \Omega_{\epsilon_{0}} \backslash D_{0}\right) \cap C\left([0,1] \times \Omega_{\epsilon_{0}}\right)$ of the Rayleigh equation (4.1). Moreover, there exists $\epsilon_{0}>0$ such that for any $\epsilon \in\left[0, \epsilon_{0}\right)$ and $(y, c) \in[0,1] \times \Omega_{\epsilon_{0}}$,

$$
\left|\phi_{1}(y, c)\right| \geq \frac{1}{2}, \quad\left|\phi_{1}(y, c)-1\right| \leq C|u(y)-c|^{2},
$$

where $\phi_{1}(y, c)=\frac{\phi(y, c)}{u(y)-c}$, and the constant $C$ may depend on $\alpha$.

The proof is based the following lemmas.

Lemma 4.6. Let $c \in D_{\epsilon_{0}}$ and $y_{c}=u^{-1}\left(c_{r}\right)$. Then there exists a solution $\phi(x, c) \in Y$ to the Rayleigh equation

Moreover, there holds

$$
\left\{\begin{array}{l}
\phi^{\prime \prime}-\alpha^{2} \phi-\frac{u^{\prime \prime}}{u-c} \phi=0 \\
\frac{\phi\left(y_{c}, c\right)}{u\left(y_{c}\right)-c}=1,\left.\quad\left(\frac{\phi(y, c)}{u(y)-c}\right)^{\prime}\right|_{y=y_{c}}=0
\end{array}\right.
$$

$$
\|\phi\|_{Y} \leq C
$$

where the constant $C$ may depend on $\alpha$. 
Proof. Let $\phi_{1}=\frac{\phi}{u-c}$, then $\phi_{1}$ satisfies

$$
\left((u-c)^{2} \phi_{1}^{\prime}\right)^{\prime}=\alpha^{2} \phi_{1}(u-c)^{2}
$$

from which, we infer that

$$
\phi_{1}(y, c)=1+\int_{y_{c}}^{y} \frac{\alpha^{2}}{\left(u\left(y^{\prime}\right)-c\right)^{2}} \int_{y_{c}}^{y^{\prime}} \phi_{1}(z, c)(u(z)-c)^{2} d z d y^{\prime} .
$$

This means that $\phi_{1}(y, c)$ satisfies

$$
\phi_{1}(y, c)=1+\alpha^{2} T \phi_{1}(y, c) .
$$

It follows from Lemma 4.4 that the operator $I-\alpha^{2} T$ is invertible in the space $Y$. Thus,

$$
\phi_{1}(y, c)=\left(I-\alpha^{2} T\right)^{-1} 1
$$

with the bound $\left\|\phi_{1}\right\|_{Y} \leq C$, hence $\|\phi\|_{Y} \leq C$.

In a similar way as in Lemma 4.6, we can show that

Lemma 4.7. Let $c \in B_{\epsilon_{0}}^{l}$. Then there exists a solution $\phi(x, c) \in Y_{l}$ to the Rayleigh equation

$$
\left\{\begin{array}{l}
\phi^{\prime \prime}-\alpha^{2} \phi-\frac{u^{\prime \prime}}{u-c} \phi=0, \\
\frac{\phi(0, c)}{u(0)-c}=1,\left.\quad\left(\frac{\phi(y, c)}{u(y)-c}\right)^{\prime}\right|_{y=0}=0 .
\end{array}\right.
$$

Moreover, there holds

$$
\|\phi\|_{Y_{l}} \leq C
$$

where the constant $C$ may depend on $\alpha$.

Lemma 4.8. Let $c \in B_{\epsilon_{0}}^{r}$. Then there exists a solution $\phi(x, c) \in Y_{r}$ to the Rayleigh equation

$$
\left\{\begin{array}{l}
\phi^{\prime \prime}-\alpha^{2} \phi-\frac{u^{\prime \prime}}{u-c} \phi=0, \\
\frac{\phi(1, c)}{u(1)-c}=1,\left.\quad\left(\frac{\phi(y, c)}{u(y)-c}\right)^{\prime}\right|_{y=1}=0 .
\end{array}\right.
$$

Moreover, there holds

$$
\|\phi\|_{Y_{r}} \leq C
$$

where the constant $C$ may depend on $\alpha$.

Lemma 4.9. Let $c \in D_{0}$ and $y_{c}=u^{-1}(c)$. Then there exists a solution $\phi(y, c) \in Y_{C}$ to the Rayleigh equation

$$
\left\{\begin{array}{l}
\phi^{\prime \prime}-\alpha^{2} \phi-\frac{u^{\prime \prime}}{u-c} \phi=0, \\
\phi\left(y_{c}, c\right)=0, \quad \phi^{\prime}\left(y_{c}, c\right)=u^{\prime}\left(y_{c}\right) .
\end{array}\right.
$$

Moreover, there holds

$$
\|\phi\|_{Y_{C}} \leq C
$$

where the constant $C$ may depend on $\alpha$.

Proof. We rewrite the Rayleigh equation (4.1) as

$$
\left(\phi^{\prime}(u-c)-\phi u^{\prime}\right)^{\prime}=\alpha^{2} \phi(u-c) .
$$

Using the boundary conditions $\phi\left(x_{0}\right)=0$ and $\phi^{\prime}\left(x_{0}\right)=u^{\prime}\left(x_{0}\right)$, we get

$$
\phi^{\prime}(y)(u(y)-c)-\phi(y) u^{\prime}(x)=\int_{y_{c}}^{y} \alpha^{2} \phi(z)(u(z)-c) d z
$$


which implies

$$
\left(\frac{\phi(y, c)}{u(y)-u\left(y_{c}\right)}\right)^{\prime}=\frac{\alpha^{2}}{\left(u(y)-u\left(y_{c}\right)\right)^{2}} \int_{y_{c}}^{y} \phi(z)(u(z)-c) d z .
$$

Let $\phi_{1}(y, c)=\frac{\phi(y, c)}{u(y)-c}$. So, $\phi_{1}\left(y_{c}\right)=1$ and

$$
\phi_{1}(y, c)=1+\int_{y_{c}}^{y} \frac{\alpha^{2}}{\left(u\left(y^{\prime}\right)-u\left(y_{c}\right)\right)^{2}} \int_{y_{c}}^{y^{\prime}} \phi_{1}(z, c)\left(u(z)-u\left(y_{c}\right)\right)^{2} d z d y^{\prime},
$$

that is,

$$
\phi_{1}(y, c)=1+\alpha^{2} T \phi_{1}(y, c) .
$$

Then Lemma 4.4 ensures the existence of the solution in $Y_{C}$ to the equation (4.7) with the bound $\left\|\phi_{1}\right\|_{Y_{C}} \leq C$.

Remark 4.10. Since $T$ is a positive operator, $\phi_{1}(y, c)$ for $c \in D_{0}$ given by (4.7) satisfies

$$
\phi_{1}(y, c) \geq 1 \text {. }
$$

Moreover, the inverse of $I-\alpha^{2} T$ exists in $X_{0}$ and has the infinite series representation

$$
\left(I-\alpha^{2} T\right)^{-1}=\sum_{k=0}^{\infty} \alpha^{2 k} T^{k}
$$

Now we are in a position to prove Proposition 4.5. We define

$$
\phi(y, c) \stackrel{\text { def }}{=} \begin{cases}\phi^{0}(y, c) & \text { for } c \in D_{0} \\ \phi^{ \pm}(y, c) & \text { for } c \in D_{\epsilon_{0}} \\ \phi^{l}(y, c) & \text { for } c \in B_{\epsilon_{0}}^{l} \\ \phi^{r}(y, c) & \text { for } c \in B_{\epsilon_{0}}^{r}\end{cases}
$$

where $\phi^{ \pm}, \phi^{l}, \phi^{r}, \phi^{0}$ are given by Lemma 4.6, Lemma 4.7, Lemma 4.8 and Lemma 4.9 respectively.

By our constructions, $\phi(y, c) \in C^{1}\left([0,1] \times \Omega_{\epsilon_{0}} \backslash D_{0}\right)$. Notice that

$$
T f(y, c)=\left(y-y_{c}\right)^{2} \iint_{[0,1]^{2}} f\left(y_{c}+s t\left(y-y_{c}\right), c\right) K_{0}(s, t, y, c) d t d s
$$

where

$$
K_{0}(s, t, y, c)=s\left(\frac{u\left(y_{c}+s t\left(y-y_{c}\right), c\right)-c}{u\left(y_{c}+s\left(y-y_{c}\right), c\right)-c}\right)^{2} .
$$

Using the fact that $K_{0} \in C\left([0,1]^{3} \times \Omega_{\varepsilon_{0}}\right)$ and $\left|K_{0}\right| \leq s$, we conclude that $T$ maps $C\left([0,1] \times \Omega_{\varepsilon_{0}}\right)$ to $C\left([0,1] \times \Omega_{\varepsilon_{0}}\right)$. Then by using the formula $\phi_{1}(y, c)=\sum_{k=0}^{+\infty} \alpha^{2 k} T^{k} 1$ for $c \in \Omega_{\varepsilon_{0}}$ and that the convergence is uniform, we conclude that $\phi_{1}(y, c) \in C\left([0,1] \times \Omega_{\varepsilon_{0}}\right)$, thus $\phi(y, c) \in C([0,1] \times$ $\left.\Omega_{\epsilon_{0}}\right)$. Furthermore, Remark 4.10 ensures that there exists $\epsilon_{0}>0$ such that for any $\epsilon \in\left[0, \epsilon_{0}\right)$ and $(y, c) \in[0,1] \times \Omega_{\epsilon_{0}}$,

$$
\left|\phi_{1}(y, c)\right| \geq \frac{1}{2}
$$

Using the integral representation of $\phi_{1}(y, c)$, we have

$$
\left|\phi_{1}(y, c)-1\right| \leq \alpha^{2} \int_{y_{c}}^{y} \int_{y_{c}}^{z}\left|\phi_{1}\left(y^{\prime}, c\right)\right|\left|\frac{u\left(y^{\prime}\right)-c}{u(z)-c}\right|^{2} d y^{\prime} d z
$$


LINEAR INVISCID DAMPING FOR A CLASS OF MONOTONE SHEAR FLOW IN SOBOLEV SPACES

$$
\begin{aligned}
& \leq C\left|y-y_{c}\right|^{2} \leq C\left|u(y)-u\left(y_{c}\right)\right|^{2} \\
& \leq C|u(y)-c|^{2} .
\end{aligned}
$$

This completes the proof of Proposition 4.5.

\section{UNiform ESTIMATES FOR THE HOMOGENEOUS RAYLEIGH EQUATION}

The goal of this section is to establish some uniform estimates in wave number $\alpha$ for the solution $\phi(y, c)$ for $c \in D_{0}$ of the Rayleigh equation given by Lemma 4.9. Let $\phi_{1}(y, c)=\frac{\phi(y, c)}{u(y)-c}$ and $y_{c}=u^{-1}(c)$ for $c \in D_{0}$.

Without loss of generality, we always assume $\alpha \geq 1$ in the sequel.

\subsection{Uniform estimates in wave number.}

Proposition 5.1. There exists a constant $C$ independent of $\alpha$ such that for any $(y, c) \in$ $[0,1] \times D_{0}$,

$$
\begin{aligned}
& \frac{\sinh \alpha\left(y-y_{c}\right)}{C \alpha} \leq \phi(y, c) \leq \frac{C \sinh \alpha\left(y-y_{c}\right)}{\alpha}, \\
& \frac{\sinh \alpha\left(y-y_{c}\right)}{C \alpha\left(y-y_{c}\right)} \leq \phi_{1}(y, c) \leq \frac{C \sinh \alpha\left(y-y_{c}\right)}{\alpha\left(y-y_{c}\right)} .
\end{aligned}
$$

Moreover, it holds that

$$
\left|\partial_{y}^{\beta} \partial_{c}^{\gamma} \phi_{1}(y, c)\right| \leq C \alpha^{\beta+\gamma} \phi_{1}(y, c)
$$

for $\beta+\gamma \leq 2$.

The proof of the proposition needs the following two lemmas.

Lemma 5.2. Let the operator $S$ be defined by

$$
S f(y, c) \stackrel{d e f}{=} \int_{y_{c}}^{y} \frac{d y^{\prime}}{\sinh ^{2} \alpha\left(y^{\prime}-y_{c}\right)} \int_{y_{c}}^{y^{\prime}} \frac{\sinh ^{2} \alpha\left(z-y_{c}\right) u^{\prime \prime}(z)}{u(z)-u\left(y_{c}\right)} f(z, c) d z .
$$

Then there exists a constant $C$ independent of $\alpha$ such that

$$
\|S f\|_{L^{\infty}\left([0,1] \times D_{0}\right)} \leq C \frac{1+\ln \alpha}{\alpha}\|f\|_{L^{\infty}\left([0,1] \times D_{0}\right)} .
$$

Especially, there exists $M_{0}>0$ such that for $\alpha \geq M_{0}$,

$$
\|S f\|_{L^{\infty}\left([0,1] \times D_{0}\right)} \leq \frac{1}{2}\|f\|_{L^{\infty}\left([0,1] \times D_{0}\right)} .
$$

Proof. A direct calculation shows

$$
\begin{aligned}
\|S f\|_{L^{\infty}\left([0,1] \times D_{0}\right)} & \leq\left|\int_{y_{c}}^{y} \frac{d y^{\prime}}{\sinh ^{2} \alpha\left(y^{\prime}-y_{c}\right)} \int_{y_{c}}^{y^{\prime}} \frac{\sinh ^{2} \alpha\left(z-y_{c}\right) u^{\prime \prime}(z)}{u(z)-u\left(y_{c}\right)} f(z, c) d z\right| \\
& =\left|\int_{y_{c}}^{y} \frac{d y}{\sinh ^{2} \alpha\left(y^{\prime}-y_{c}\right)} \int_{y_{c}}^{y^{\prime}} \frac{\sinh ^{2} \alpha\left(z-y_{c}\right)}{z-y_{c}} \frac{u^{\prime \prime}(z)}{\int_{0}^{1} u^{\prime}\left(y_{c}+\left(z-y_{c}\right) t\right) d t} f(z, c) d z\right| \\
& \leq C \frac{1}{\alpha} \int_{0}^{\alpha\left|y-y_{c}\right|} \frac{d y}{\sinh ^{2} y^{\prime}} \int_{0}^{\left|y^{\prime}\right|} \frac{\sinh ^{2} z}{z} d z\|f\|_{L^{\infty}\left([0,1] \times D_{0}\right)} \\
& \leq C \frac{1}{\alpha} \int_{0}^{\alpha} \frac{\sinh ^{2} z}{z} d z \int_{|z|}^{\infty} \frac{d y^{\prime}}{\sinh ^{2} y^{\prime}}\|f\|_{L^{\infty}\left([0,1] \times D_{0}\right)}
\end{aligned}
$$




$$
\begin{aligned}
& =C \frac{1}{\alpha} \int_{0}^{\alpha} \frac{1-e^{-2 z}}{z} d z\|f\|_{L^{\infty}\left([0,1] \times D_{0}\right)} \\
& \leq C \frac{1+\ln \alpha}{\alpha}\|f\|_{L^{\infty}\left([0,1] \times D_{0}\right)} .
\end{aligned}
$$

The second statement is direct.

Lemma 5.3. Given $\alpha \geq 1$, we denote $\Sigma_{\alpha} \triangleq\left\{(y, c):\left|y-y_{c}\right| \leq \frac{1}{\alpha}, c=u\left(y_{c}\right)\right\}$. Then there exists a constant $C$ independent of $\alpha$ such that

$$
\begin{aligned}
& \left\|T_{0} f\right\|_{L^{\infty}\left(\Sigma_{\alpha}\right)} \leq \frac{1}{\alpha}\|f\|_{L^{\infty}\left(\Sigma_{\alpha}\right)}, \\
& \left\|T_{k, k} f\right\|_{L^{\infty}\left(\Sigma_{\alpha}\right)} \leq \frac{C}{\alpha}\|f\|_{L^{\infty}\left(\Sigma_{\alpha}\right)} .
\end{aligned}
$$

Moreover, for $\beta+\gamma \leq 2$,

$$
\left\|\partial_{y}^{\beta} \partial_{c}^{\gamma} T_{k, k+1} f\right\|_{L^{\infty}\left(\Sigma_{\alpha}\right)} \leq C \sum_{\beta_{1}+\gamma_{1} \leq \beta+\gamma}\left\|\partial_{x}^{\beta_{1}} \partial_{c}^{\gamma_{1}} f\right\|_{L^{\infty}\left(\Sigma_{\alpha}\right)} .
$$

Proof. For $(y, c) \in \Sigma_{\alpha}$, we have

$$
\left|T_{0} f(y, c)\right|=\left|\int_{y_{c}}^{y} f(z, c) d z\right| \leq\left|y-y_{c}\right|\|f\|_{L^{\infty}\left(\Sigma_{\alpha}\right)} \leq \frac{1}{\alpha}\|f\|_{L^{\infty}\left(\Sigma_{\alpha}\right)},
$$

and

$$
\begin{aligned}
\left|T_{k, k} f(y, c)\right| & =\left|\frac{1}{(u(y)-c)^{k}} \int_{y_{c}}^{y}(u(z)-c)^{k} f(z, c) d z\right| \\
& \leq C\left|y-y_{c}\right|\left\|u^{\prime}\right\|_{L^{\infty}}^{k}\|f\|_{L^{\infty}\left(\Sigma_{\alpha}\right)} \leq \frac{C}{\alpha}\|f\|_{L^{\infty}\left(\Sigma_{\alpha}\right)} .
\end{aligned}
$$

Using the formula

$$
T_{k, k+1} f(y, c)=\int_{0}^{1} f\left(y_{c}+t\left(y-y_{c}\right), c\right) \frac{\left(\int_{0}^{1} u^{\prime}\left(y_{c}+s t\left(y-y_{c}\right)\right) d s\right)^{k}}{\left(\int_{0}^{1} u^{\prime}\left(y_{c}+s\left(y-y_{c}\right)\right) d s\right)^{k+1}} t^{k} d t,
$$

a direct calculation gives the third inequality of the lemma. We omit the details here.

Now let us turn to the proof of Proposition 5.1.

Proof. Let $M_{0}$ be given by Lemma 5.2. The case of $\alpha \leq M_{0}$ is a direct consequence of Lemma 4.9 , which implies that

$$
1 \leq\left|\phi_{1}(y, c)\right| \leq C, \quad C^{-1}\left|y-y_{c}\right| \leq|\phi(y, c)| \leq C\left|y-y_{c}\right|, \quad\left|\partial_{y}^{\beta} \partial_{c}^{\gamma} \phi_{1}(y, c)\right| \leq C
$$

for any $(y, c) \in[0,1] \times D_{0}$, where the constant $C$ depends on $M_{0}$.

Thus, it suffices to consider the case of $\alpha \geq M_{0}$. It is easy to check that the solution $\phi(y, c)$ satisfies

$$
\left(\sinh ^{2} \alpha\left(y-y_{c}\right)\left(\frac{\phi(y, c)}{\sinh \alpha\left(y-y_{c}\right)}\right)^{\prime}\right)^{\prime}=\sinh \alpha\left(y-y_{c}\right) \frac{u^{\prime \prime}}{u(y)-u\left(y_{c}\right)} \phi(y, c) .
$$

Let $\widetilde{\phi}_{1}(y, c)=\frac{\alpha \phi(y, c)}{\sinh \alpha\left(y-y_{c}\right)}$, then $\widetilde{\phi}_{1}(y, c)$ satisfies

$$
\widetilde{\phi}_{1}(y, c)=u^{\prime}\left(y_{c}\right)+\int_{y_{c}}^{y} \frac{d y^{\prime}}{\sinh ^{2} \alpha\left(y^{\prime}-y_{c}\right)} \int_{y_{c}}^{y^{\prime}} \frac{\sinh ^{2} \alpha\left(z-y_{c}\right) u^{\prime \prime}(z)}{u(z)-u\left(y_{c}\right)} \widetilde{\phi}_{1}(z, c) d z .
$$


LINEAR INVISCID DAMPING FOR A CLASS OF MONOTONE SHEAR FLOW IN SOBOLEV SPACES 13

Lemma 5.2 ensures that

$$
\widetilde{\phi}_{1}(y, c)=(I-S)^{-1}\left(u^{\prime}\left(y_{c}\right)\right)
$$

with the bound

$$
C^{-1} \leq \widetilde{\phi}_{1}(y, c) \leq C \quad \text { for any } \quad(y, c) \in[0,1] \times D_{0},
$$

which implies the first part of the proposition.

Next we prove the derivative estimates. Thanks to $u^{\prime}(y) \geq c_{0}$, it holds that for any $y, z$ with $\left|y-y_{c}\right| \leq\left|z-y_{c}\right|$

$$
(u(y)-c)^{2} \leq(u(z)-c)^{2}, \quad c=u\left(y_{c}\right)
$$

Thus, for $(y, c) \in \Sigma_{\alpha}$,

$$
\begin{aligned}
|T f(y, c)| & \leq \int_{y_{c}}^{y} \frac{1}{(u(z)-c)^{2}} \int_{y_{c}}^{z}\left(u\left(y^{\prime}\right)-c\right)^{2}\|f\|_{L^{\infty}\left(\Sigma_{\alpha}\right)} d y^{\prime} d z \\
& \leq \int_{y_{c}}^{y}\left(z-y_{c}\right) d z\|f\|_{L^{\infty}\left(\Sigma_{\alpha}\right)} \\
& \leq \frac{1}{2 \alpha^{2}}\|f\|_{L^{\infty}\left(\Sigma_{\alpha}\right)} .
\end{aligned}
$$

This means that $\alpha^{2} T$ is a contraction mapping from $L^{\infty}\left(\Sigma_{\alpha}\right)$ to $L^{\infty}\left(\Sigma_{\alpha}\right)$. Thus,

$$
\left\|\phi_{1}\right\|_{L^{\infty}\left(\Sigma_{\alpha}\right)} \leq\left\|\left(I-\alpha^{2} T\right)^{-1} 1\right\|_{L^{\infty}\left(\Sigma_{\alpha}\right)} \leq 2 .
$$

On the other hand, we have

$$
\begin{aligned}
& \partial_{y} \phi_{1}(y, c)=\alpha^{2} T_{2,2} \phi_{1}(y, c), \\
& \partial_{y y} \phi_{1}(y, c)=-2 \alpha^{2} u^{\prime}(y) T_{2,3} \phi_{1}(y, c)+\phi(y, c),
\end{aligned}
$$

then it follows from Lemma 5.3 that for any $(y, c) \in \Sigma_{\alpha}$,

$$
\left|\partial_{y} \phi_{1}(y, c)\right| \leq C \alpha, \quad\left|\partial_{y y} \phi_{1}(y, c)\right| \leq C \alpha^{2} .
$$

Now we deal with the derivative estimate in $c$ variable. Recalling that

$$
\begin{aligned}
& \phi_{1}(y, c)=1+\alpha^{2} T \phi_{1}(y, c), \\
& \partial_{c} T f=2 T_{0} T_{2,3} f-2 T_{0} T_{1,2} f+T \partial_{c} f,
\end{aligned}
$$

we obtain

$$
\begin{aligned}
& \partial_{c} \phi_{1}(y, c)=2 \alpha^{2} T_{0} T_{2,3} \phi_{1}(y, c)-2 \alpha^{2} T_{0} T_{1,2} \phi_{1}(y, c)+\alpha^{2} T \partial_{c} \phi_{1}(y, c), \\
& \partial_{y} \partial_{c} \phi_{1}(y, c)=2 \alpha^{2} T_{2,3} \phi_{1}(y, c)-2 \alpha^{2} T_{1,2} \phi_{1}(y, c)+\alpha^{2} T_{2,2} \partial_{c} \phi_{1}(y, c),
\end{aligned}
$$

and

$$
\begin{aligned}
\partial_{c}^{2} \phi_{1}(y, c)= & 2 \alpha^{2} \partial_{c} T_{0} T_{2,3} \phi_{1}(y, c)-2 \alpha^{2} \partial_{c} T_{0} T_{1,2} \phi_{1}(y, c)+\alpha^{2} \partial_{c} T \partial_{c} \phi_{1}(y, c) \\
= & 2 \alpha^{2} T_{0} \partial_{c} T_{2,3} \phi_{1}(y, c)-2 \alpha^{2} T_{0} \partial_{c} T_{1,2} \phi_{1}(y, c)+\frac{\alpha^{2}}{3 u^{\prime}\left(y_{c}\right)} \\
& +2 \alpha^{2} T_{0} T_{2,3} \partial_{c} \phi_{1}(y, c)-2 \alpha^{2} T_{0} T_{1,2} \partial_{c} \phi_{1}(y, c)+\alpha^{2} T \partial_{c}^{2} \phi_{1}(y, c),
\end{aligned}
$$

which along with Lemma 5.3 ensure that

$$
\begin{aligned}
\left\|\partial_{c} \phi_{1}\right\|_{L^{\infty}\left(\Sigma_{\alpha}\right)} & =\left\|\left(I-\alpha^{2} T\right)^{-1}\left(2 \alpha^{2} T_{0} T_{2,3} \phi_{1}(y, c)-2 \alpha^{2} T_{0} T_{1,2} \phi_{1}\right)\right\|_{L^{\infty}\left(\Sigma_{\alpha}\right)} \\
& \leq C\left(\alpha^{2}\left\|T_{0} T_{2,3} \phi_{1}\right\|_{L^{\infty}\left(\Sigma_{\alpha}\right)}+\alpha^{2}\left\|T_{0} T_{1,2} \phi_{1}\right\|_{L^{\infty}\left(\Sigma_{\alpha}\right)}\right) \\
& \leq C \alpha\left\|\phi_{1}\right\|_{L^{\infty}\left(\Sigma_{\alpha}\right)} \leq C \alpha
\end{aligned}
$$


and

$$
\left\|\partial_{y} \partial_{c} \phi_{1}\right\|_{L^{\infty}\left(\Sigma_{\alpha}\right)}+\left\|\partial_{c}^{2} \phi_{1}\right\|_{L^{\infty}\left(\Sigma_{\alpha}\right)} \leq C \alpha^{2} .
$$

Finally, let us consider the case of $\left|y-y_{c}\right| \geq \frac{1}{\alpha}$. Using the fact that

$$
\begin{aligned}
& \left|\phi_{1}(y, c)\right| \leq C \frac{\sinh \alpha\left(y-y_{c}\right)}{\alpha\left(y-y_{c}\right)} \leq C \cosh \alpha\left(y-y_{c}\right), \\
& C \sinh \alpha\left|y-y_{c}\right| \geq \cosh \alpha\left(y-y_{c}\right)-1 \quad \text { for } \quad\left|y-y_{c}\right| \geq \frac{1}{\alpha},
\end{aligned}
$$

we infer that

$$
\begin{aligned}
\left|\partial_{y} \phi_{1}(y, c)\right| & =\left|\frac{\alpha^{2}}{\left(u(y)-u\left(y_{c}\right)\right)^{2}} \int_{y_{c}}^{y} \phi_{1}(z)\left(u(z)-u\left(y_{c}\right)\right)^{2} d z\right| \\
& \leq C\left|\frac{\alpha}{\left(y-y_{c}\right)^{2}} \int_{y_{c}}^{y} \sinh \alpha\left(z-y_{c}\right)\left(z-y_{c}\right) d z\right| \\
& \leq C \frac{\left|\cosh \alpha\left(y-y_{c}\right)-1\right|}{\left|y-y_{c}\right|} \leq C \alpha \phi_{1}(y, c) .
\end{aligned}
$$

Using the equation

$$
\phi_{1}^{\prime \prime}+\frac{2 u^{\prime}}{u-c} \phi_{1}^{\prime}=\alpha^{2} \phi_{1}
$$

we obtain

$$
\left|\partial_{y}^{2} \phi_{1}\right| \leq C \alpha^{2} \phi_{1}
$$

To estimate the derivative in $c$ variable, we introduce $f=\frac{\partial_{y} \phi_{1}}{\phi_{1}}$ and $g=\frac{\partial_{c} \phi_{1}}{\phi_{1}}$. It is easy to see that $\partial_{c} f=\partial_{y} g$ and

$$
\left(\phi^{2} \partial_{c} f\right)^{\prime}+2 \phi_{1}^{2} u^{\prime} f=0
$$

which gives

$$
\partial_{c} f(y, c)=\partial_{y} g(y, c)=\frac{-2}{\phi(y, c)^{2}} \int_{y_{c}}^{y} \phi_{1}^{2}(z, c) u^{\prime}(z) f(z, c) d z=\frac{-2}{\phi(y, c)^{2}} T_{0}\left(\phi_{1}^{2} u^{\prime} f\right),
$$

For $\left|y-y_{c}\right| \geq \frac{1}{\alpha}, \phi_{1}^{2}-1 \sim \phi_{1}^{2}$ and $\partial_{y} \phi_{1}(y, c)$ has the same sign as $y-y_{c}$. Then

$$
\begin{aligned}
\left|\partial_{y} g(y, c)\right|=\left|\partial_{c} f(y, c)\right| & \leq \frac{C}{\phi(y, c)^{2}} \int_{y_{c}}^{y} \phi_{1}(z, c) \partial_{z} \phi_{1}(z, c) d z \\
& \leq C \frac{\phi_{1}(y, c)^{2}-1}{\phi(y, c)^{2}} \leq \frac{C}{\left|y-y_{c}\right|^{2}},
\end{aligned}
$$

from which, it follows that

$$
|g(y, c)| \leq \frac{C}{\left|y-y_{c}\right|} \leq C \alpha
$$

and thus,

$$
\left|\partial_{c} \phi_{1}\right| \leq C \alpha \phi_{1}, \quad\left|\partial_{y} \partial_{c} \phi_{1}\right| \leq C \alpha^{2} \phi_{1}
$$

We have

$$
\partial_{c}^{2} f(y, c)=\partial_{y} \partial_{c} g(y, c)=-2 \int_{y_{c}}^{y} \partial_{c}\left(\frac{\phi_{1}(z, c)^{2}}{\phi(y, c)^{2}} f(z, c)\right) u^{\prime}(z) d z
$$


which implies

$$
\left|\partial_{c}^{2} f(y, c)\right| \leq \frac{C \alpha}{\left|y-y_{c}\right|^{2}}
$$

Thus, we obtain

$$
\left|\partial_{c} g(y, c)\right| \leq \frac{C \alpha}{\left|y-y_{c}\right|} \leq C \alpha^{2}, \quad\left|\partial_{c}^{2} \phi_{1}\right| \leq C \alpha^{2} \phi_{1} .
$$

The proof is finished.

Lemma 5.4. There exists a constant $C$ independent of $\alpha$ such that for any $(y, c) \in[0,1] \times D_{0}$,

$$
\begin{gathered}
\frac{1}{C}\left(\frac{\sinh \alpha\left(y-y_{c}\right)}{\alpha\left(y-y_{c}\right)}-1\right) \leq \phi_{1}(y, c)-1 \leq C\left(\frac{\sinh \alpha\left(y-y_{c}\right)}{\alpha\left(y-y_{c}\right)}-1\right), \\
\left|\phi_{1}(y, c)-1\right| \leq C \alpha^{2}\left(y-y_{c}\right)^{2} \phi_{1}(y, c),
\end{gathered}
$$

where $c=u\left(y_{c}\right)$.

Proof. We have

$$
\begin{aligned}
\phi_{1}(y, c)-1 & =\int_{y_{c}}^{y} \frac{\alpha^{2}}{\left(u\left(y^{\prime}\right)-u\left(y_{c}\right)\right)^{2}} \int_{y_{c}}^{y^{\prime}} \phi_{1}(z)\left(u(z)-u\left(y_{c}\right)\right)^{2} d z d y^{\prime} \\
& \geq C^{-1} \int_{y_{c}}^{y} \frac{\alpha}{\left(y^{\prime}-y_{c}\right)^{2}} \int_{y_{c}}^{y^{\prime}} \sinh \alpha\left(z-y_{c}\right)\left(z-y_{c}\right) d z d y^{\prime} \\
& =C^{-1} \int_{0}^{\alpha\left(y-y_{c}\right)} \frac{\cosh z}{z}-\frac{\sinh z}{z^{2}} d z \\
& =C^{-1}\left(\frac{\sinh \alpha\left(y-y_{c}\right)}{\alpha\left(y-y_{c}\right)}-1\right) \geq 0,
\end{aligned}
$$

and

$$
\begin{aligned}
\phi_{1}(y, c)-1 & =\int_{y_{c}}^{y} \frac{\alpha^{2}}{\left(u\left(y^{\prime}\right)-u\left(y_{c}\right)\right)^{2}} \int_{y_{c}}^{y^{\prime}} \phi_{1}(z)\left(u(z)-u\left(y_{c}\right)\right)^{2} d z d y^{\prime} \\
& \leq C \int_{y_{c}}^{y} \frac{\alpha}{\left(y^{\prime}-y_{c}\right)^{2}} \int_{y_{c}}^{y^{\prime}} \sinh \alpha\left(z-y_{c}\right)\left(z-y_{c}\right) d z d y^{\prime} \\
& =C \int_{0}^{\alpha\left(y-y_{c}\right)} \frac{\cosh z}{z}-\frac{\sinh z}{z^{2}} d z \\
& =C\left(\frac{\sinh \alpha\left(y-y_{c}\right)}{\alpha\left(y-y_{c}\right)}-1\right) .
\end{aligned}
$$

This gives the first inequality.

Thanks to $\partial_{y} \phi_{1}(y, c) \geq 0$ for $y \geq y_{c}$ and $\partial_{y} \phi_{1}(y, c) \leq 0$ for $y \leq y_{c}$, we have

$$
\phi_{1}(y, c) \geq \phi_{1}(z, c), \quad|u(z)-c| \leq|u(y)-c|
$$

for $\left|y-y_{c}\right| \geq\left|z-y_{c}\right|$. Then we get

$$
\begin{aligned}
\left|\phi_{1}(y, c)-1\right| & \leq \alpha^{2} \int_{y_{c}}^{y} \int_{y_{c}}^{z} \phi_{1}\left(y^{\prime}, c\right) \frac{\left(u\left(y^{\prime}\right)-c\right)^{2}}{(u(z)-c)^{2}} d y^{\prime} d z \\
& \leq C \alpha^{2}\left|y-y_{c}\right|^{2} \phi_{1}(y, c) \leq C \alpha^{2}\left|u(y)-u\left(y_{c}\right)\right|^{2} \phi_{1}(y, c) .
\end{aligned}
$$

The second inequality is proved. 
Remark 5.5. We also need a more precise estimate for $\phi_{1}(y, c)-1$ :

$$
\begin{aligned}
\phi_{1}(y, c)-1 & =\alpha^{2}\left(y-y_{c}\right)^{2} \iint_{[0,1]^{2}} \phi_{1}\left(y_{c}+s t\left(y-y_{c}\right), c\right) K\left(y_{c}+s\left(y-y_{c}\right), y_{c}, t\right) s d t d s \\
& =\alpha^{2}\left(y-y_{c}\right)^{2} \mathcal{T}\left(\phi_{1}\right)(y, c),
\end{aligned}
$$

where $y_{c}=u^{-1}(c)$ and

$$
K\left(y, y_{c}, t\right)=\frac{\left(\int_{0}^{1} u^{\prime}\left(y_{c}+s t\left(y-y_{c}\right)\right) d s\right)^{2}}{\left(\int_{0}^{1} u^{\prime}\left(y_{c}+s\left(y-y_{c}\right)\right) d s\right)^{2}} t^{2} .
$$

Using the fact that $\|K\|_{C_{y, y_{c}, t}^{2}} \leq C$ and $\phi_{1}(y, c) \geq \phi_{1}(z, c)$ for $\left|y-y_{c}\right| \geq\left|z-y_{c}\right|$, we get by Proposition 5.1 that

$$
\begin{aligned}
& \left|\mathcal{T}\left(\phi_{1}\right)(y, c)\right| \leq C \phi_{1}(y, c), \\
& \left|\partial_{y} \mathcal{T}\left(\phi_{1}\right)(y, c)\right|+\left|\partial_{c} \mathcal{T}\left(\phi_{1}\right)(y, c)\right| \leq C \alpha \phi_{1}(y, c), \\
& \left|\partial_{y}^{2} \mathcal{T}\left(\phi_{1}\right)(y, c)\right|+\left|\partial_{c}^{2} \mathcal{T}\left(\phi_{1}\right)(y, c)\right|+\left|\partial_{y c} \mathcal{T}\left(\phi_{1}\right)(y, c)\right| \leq C \alpha^{2} \phi_{1}(y, c) .
\end{aligned}
$$

\subsection{Uniform estimates for good derivative.}

Proposition 5.6. There exists a constant $C$ independent of $\alpha$ such that for any $(z, c) \in$ $D_{0} \times D_{0}$,

$$
\begin{aligned}
& \left|\left(\partial_{z}+\partial_{c}\right) \phi_{1}\left(u^{-1}(z), c\right)\right| \leq C \alpha^{2}\left(y-y_{c}\right)^{2} \phi_{1}, \\
& \left|\left(\partial_{z}+\partial_{c}\right)^{2} \phi_{1}\left(u^{-1}(z), c\right)\right| \leq C \alpha^{2}\left(y-y_{c}\right)^{2} \cosh \alpha\left(y-y_{c}\right),
\end{aligned}
$$

where $y_{c}=u^{-1}(c)$ and $y=u^{-1}(z)$.

Proof. A direct calculation gives

$$
\begin{aligned}
& \left(\partial_{z}+\partial_{c}\right) \phi_{1}\left(u^{-1}(z), c\right) \\
= & \frac{\alpha^{2}}{(z-c)^{2}} \int_{y_{c}}^{u^{-1}(z)}\left(u\left(y^{\prime}\right)-c\right)^{2} \phi_{1}\left(y^{\prime}, c\right) d y^{\prime} \frac{1}{u^{\prime}\left(u^{-1}(z)\right)} \\
& +2 \alpha^{2} \int_{y_{c}}^{u^{-1}(z)} \frac{1}{\left(u\left(y^{\prime}\right)-c\right)^{3}} \int_{y_{c}}^{y^{\prime}}(u(t)-c)^{2} \phi_{1}(t, c) d t d y^{\prime} \\
& -2 \alpha^{2} \int_{y_{c}}^{u^{-1}(z)} \frac{1}{\left(u\left(y^{\prime}\right)-c\right)^{2}} \int_{y_{c}}^{y^{\prime}}(u(t)-c) \phi_{1}(t, c) d t d y^{\prime} \\
& +\alpha^{2} T\left(\partial_{c} \phi_{1}\right)\left(u^{-1}(z), c\right) \\
= & \frac{\alpha^{2}}{(z-c)^{2}} \int_{y_{c}}^{u^{-1}(z)}\left(u\left(y^{\prime}\right)-c\right)^{2} \phi_{1}\left(y^{\prime}, c\right) d y^{\prime} \frac{1}{u^{\prime}\left(u^{-1}(z)\right)}+\alpha^{2} T\left(\partial_{c} \phi_{1}\right)\left(u^{-1}(z), c\right) \\
& -\alpha^{2} \int_{y_{c}}^{u^{-1}(z)} \partial_{y^{\prime}}\left(\frac{1}{\left(u\left(y^{\prime}\right)-c\right)^{2}}\right) \frac{1}{u^{\prime}\left(y^{\prime}\right)} \int_{y_{c}}^{y^{\prime}}(u(t)-c)^{2} \phi_{1}(t, c) d t d y^{\prime} \\
& -\alpha^{2} \int_{y_{c}}^{u^{-1}(z)} \frac{1}{\left(u\left(y^{\prime}\right)-c\right)^{2}} \int_{y_{c}}^{y^{\prime}} \partial_{t}\left((u(t)-c)^{2}\right) \frac{\phi_{1}(t, c)}{u^{\prime}(t)} d t d y^{\prime},
\end{aligned}
$$

Integration by parts and taking into consideration the boundary condition, we obtain

$$
\left(\partial_{z}+\partial_{c}\right) \phi_{1}\left(u^{-1}(z), c\right)
$$


LINEAR INVISCID DAMPING FOR A CLASS OF MONOTONE SHEAR FLOW IN SOBOLEV SPACES 17

$$
\begin{aligned}
= & \frac{\alpha^{2}}{(z-c)^{2}} \int_{y_{c}}^{u^{-1}(z)}\left(u\left(y^{\prime}\right)-c\right)^{2} \phi_{1}\left(y^{\prime}, c\right) d y^{\prime} \frac{1}{u^{\prime}\left(u^{-1}(z)\right)}+\alpha^{2} T\left(\partial_{c} \phi_{1}\right)\left(u^{-1}(z), c\right) \\
& -\left.\alpha^{2}\left(\frac{1}{\left(u\left(y^{\prime}\right)-c\right)^{2}}\right) \frac{1}{u^{\prime}\left(y^{\prime}\right)} \int_{y_{c}}^{y^{\prime}}\left(u\left(z^{\prime}\right)-c\right)^{2} \phi_{1}\left(z^{\prime}, c\right) d z^{\prime}\right|_{y_{c}} ^{u^{-1}(z)} \\
& +\alpha^{2} \int_{y_{c}}^{u^{-1}(z)}\left(\frac{1}{\left(u\left(y^{\prime}\right)-c\right)^{2}}\right) \partial_{y^{\prime}}\left(\frac{1}{u^{\prime}\left(y^{\prime}\right)} \int_{y_{c}}^{y^{\prime}}\left(u\left(z^{\prime}\right)-c\right)^{2} \phi_{1}\left(z^{\prime}, c\right) d z^{\prime}\right) d y^{\prime} \\
& -\left.\alpha^{2} \int_{y_{c}}^{u^{-1}(z)} \frac{1}{\left(u\left(y^{\prime}\right)-c\right)^{2}}\left(u\left(z^{\prime}\right)-c\right)^{2} \frac{\phi_{1}\left(z^{\prime}, c\right)}{u^{\prime}\left(z^{\prime}\right)}\right|_{y_{c}} ^{y^{\prime}} d y^{\prime} \\
& +\alpha^{2} \int_{y_{c}}^{u^{-1}(z)} \frac{1}{\left(u\left(y^{\prime}\right)-c\right)^{2}} \int_{y_{c}}^{y^{\prime}}\left(u\left(z^{\prime}\right)-c\right)^{2} \partial_{y}\left(\frac{\phi_{1}\left(z^{\prime}, c\right)}{u^{\prime}\left(z^{\prime}\right)}\right) d z^{\prime} d y^{\prime} \\
= & \alpha^{2} T\left(\partial_{c} \phi_{1}\right)\left(u^{-1}(z), c\right)+\alpha^{2} \int_{y_{c}}^{u^{-1}(z)} \frac{1}{\left(u\left(y^{\prime}\right)-c\right)^{2}} \partial_{y^{\prime}}\left(\frac{1}{u^{\prime}\left(y^{\prime}\right)}\right) \int_{y_{c}}^{y^{\prime}}\left(u\left(z^{\prime}\right)-c\right)^{2} \phi_{1}\left(z^{\prime}, c\right) d z^{\prime} d y^{\prime} \\
& +\alpha^{2} \int_{y_{c}}^{u^{-1}(z)} \frac{1}{\left(u\left(y^{\prime}\right)-c\right)^{2}} \int_{y_{c}}^{y^{\prime}}\left(u\left(z^{\prime}\right)-c\right)^{2} \partial_{y}\left(\frac{\phi_{1}\left(z^{\prime}, c\right)}{u^{\prime}\left(z^{\prime}\right)}\right) d z^{\prime} d y^{\prime} \\
= & \alpha^{2} T\left(\partial_{c} \phi_{1}\right)\left(u^{-1}(z), c\right)+\alpha^{2} T_{0}\left(\partial_{y}\left(\frac{1}{u^{\prime}}\right) T_{2,2}\left(\phi_{1}\right)\right)\left(u^{-1}(z), c\right) \\
& +\alpha^{2} T\left(\partial_{y}\left(\frac{\phi_{1}}{u^{\prime}}\right)\right)\left(u^{-1}(z), c\right) \\
= & \alpha^{2} T\left(\partial_{c} \phi_{1}+\frac{1}{u^{\prime}} \partial_{y} \phi_{1}\right)\left(u^{-1}(z), c\right)+\alpha^{2} T_{0}\left(\partial_{y}\left(\frac{1}{u^{\prime}}\right) T_{2,2}\left(\phi_{1}\right)\right)\left(u^{-1}(z), c\right) \\
& +\alpha^{2} T\left(\phi_{1} \partial_{y}\left(\frac{1}{u^{\prime}}\right)\right)\left(u^{-1}(z), c\right) .
\end{aligned}
$$

This shows that

$$
\begin{aligned}
& \left(I-\alpha^{2} T\right)\left(\partial_{z} \phi_{1}+\partial_{c} \phi_{1}\right)\left(u^{-1}(z), c\right) \\
& =\alpha^{2} T_{0}\left(\partial_{y}\left(\frac{1}{u^{\prime}}\right) T_{2,2}\left(\phi_{1}\right)\right)\left(u^{-1}(z), c\right)+\alpha^{2} T\left(\phi_{1} \partial_{y}\left(\frac{1}{u^{\prime}}\right)\right)\left(u^{-1}(z), c\right),
\end{aligned}
$$

where the right hand side is bounded by $\alpha^{2} T \phi_{1}$. Recall that $T$ is a positive operator. Thus, we have

$$
\begin{aligned}
& \left|\left(\partial_{z}+\partial_{c}\right) \phi_{1}\left(u^{-1}(z), c\right)\right| \\
\leq & \mid\left(I-\alpha^{2} T\right)^{-1}\left[\alpha^{2} T_{0}\left(\partial_{y}\left(\frac{1}{u^{\prime}}\right) T_{2,2}\left(\phi_{1}\right)\right)\left(u^{-1}(z), c\right)\right. \\
& \left.+\alpha^{2} T\left(\phi_{1} \partial_{y}\left(\frac{1}{u^{\prime}}\right)\right)\left(u^{-1}(z), c\right)\right] \mid \\
\leq & C\left|\left(I-\alpha^{2} T\right)^{-1} \alpha^{2} T \phi_{1}\right| \\
\leq & C\left|\alpha^{2} T\left(I-\alpha^{2} T\right)^{-2} 1\right| \leq C\left|\sum_{k=1}^{\infty} k \alpha^{2 k} T^{k} 1\right| .
\end{aligned}
$$

Using the fact that $(u(z)-c)^{2} \leq\left(u\left(y^{\prime}\right)-c\right)^{2}$ for $\left|z-y_{c}\right| \leq\left|y^{\prime}-y_{c}\right|$, we obtain

$$
|T 1|=\left|\int_{y_{c}}^{y} \frac{1}{\left(u\left(y^{\prime}\right)-c\right)^{2}} \int_{y_{c}}^{y^{\prime}}(u(z)-c)^{2} d z d y^{\prime}\right|
$$




$$
\leq \frac{1}{2}\left(y-y_{c}\right)^{2}
$$

A simple iteration gives

$$
\left|T^{k} 1\right| \leq \frac{1}{(2 k) !}\left(y-y_{c}\right)^{2 k}
$$

which implies

$$
\begin{aligned}
\left|\sum_{k=1}^{\infty} k \alpha^{2 k} T^{k} 1\right| & \leq C \alpha\left(y-y_{c}\right) \sum_{k=1}^{\infty} \frac{1}{(2 k-1) !}\left(\alpha\left(y-y_{c}\right)\right)^{2 k-1} \\
& \leq C \alpha\left(y-y_{c}\right) \sinh \alpha\left(y-y_{c}\right) \\
& \leq C \alpha^{2}\left(y-y_{c}\right)^{2} \phi_{1},
\end{aligned}
$$

which together with (5.1) gives the first inequality.

Now we prove the second inequality. We have

$$
\begin{aligned}
& \left(\partial_{z}+\partial_{c}\right)^{2} \phi_{1}\left(u^{-1}(z), c\right) \\
& =\alpha^{2}\left(\partial_{z}+\partial_{c}\right) T\left(\partial_{c} \phi_{1}\right)\left(u^{-1}(z), c\right)+\alpha^{2}\left(\partial_{z}+\partial_{c}\right) T_{0}\left(\partial_{y}\left(\frac{1}{u^{\prime}}\right) T_{2,2}\left(\phi_{1}\right)\right)\left(u^{-1}(z), c\right) \\
& \quad+\alpha^{2}\left(\partial_{z}+\partial_{c}\right) T\left(\partial_{y}\left(\frac{\phi_{1}}{u^{\prime}}\right)\right)\left(u^{-1}(z), c\right) .
\end{aligned}
$$

Let $F(y, c)$ be a function with $\frac{F(y, c)}{(u(y)-c)^{2}} \rightarrow 0$ as $y \rightarrow y_{c}$. Then we have

$$
\begin{aligned}
\left(\partial_{z}+\partial_{c}\right) \int_{y_{c}}^{u^{-1}(z)} \frac{1}{\left(u\left(y^{\prime}\right)-c\right)^{2}} F\left(y^{\prime}, c\right) d y^{\prime} \\
=\frac{F\left(u^{-1}(z), c\right)}{(z-c)^{2}}\left(u^{-1}\right)^{\prime}(z)-\left.\frac{F\left(y^{\prime}, c\right)}{\left(u\left(y^{\prime}\right)-c\right)^{2} u^{\prime}\left(y^{\prime}\right)}\right|_{y_{c}} ^{u^{-1}(z)} \\
\quad+\int_{y_{c}}^{u^{-1}(z)} \frac{1}{\left(u\left(y^{\prime}\right)-c\right)^{2}}\left(\partial_{y}\left(\frac{1}{u^{\prime}\left(y^{\prime}\right)}\right) F\left(y^{\prime}, c\right)\right) d y^{\prime}+\int_{y_{c}}^{u^{-1}(z)} \frac{1}{\left(u\left(y^{\prime}\right)-c\right)^{2}}\left(\frac{1}{u^{\prime}\left(y^{\prime}\right)} \partial_{y} F\left(y^{\prime}, c\right)\right) d y^{\prime} \\
\quad+\int_{y_{c}}^{u^{-1}(z)} \frac{1}{\left(u\left(y^{\prime}\right)-c\right)^{2}} \partial_{c} F\left(y^{\prime}, c\right) d y^{\prime} \\
=\int_{y_{c}}^{u^{-1}(z)} \frac{F\left(y^{\prime}, c\right)}{\left(u\left(y^{\prime}\right)-c\right)^{2}} \partial_{y}\left(\frac{1}{u^{\prime}\left(y^{\prime}\right)}\right) d y^{\prime}+\int_{y_{c}}^{u^{-1}(z)} \frac{1}{\left(u\left(y^{\prime}\right)-c\right)^{2}}\left(\frac{1}{u^{\prime}\left(y^{\prime}\right)} \partial_{y} F\left(y^{\prime}, c\right)\right) d y^{\prime} \\
\quad+\int_{y_{c}}^{u^{-1}(z)} \frac{1}{\left(u\left(y^{\prime}\right)-c\right)^{2}} \partial_{c} F\left(y^{\prime}, c\right) d y^{\prime} .
\end{aligned}
$$

Similarly, we have

$$
\begin{aligned}
& \left(\partial_{z}+\partial_{c}\right) \int_{y_{c}}^{u^{-1}(z)} f\left(y^{\prime}, c\right)\left(u\left(y^{\prime}\right)-c\right)^{2} d y^{\prime} \\
& =f\left(u^{-1}(z), c\right)(z-c)^{2}\left(u^{-1}\right)^{\prime}(z)+\int_{y_{c}}^{u^{-1}(z)} \partial_{c} f\left(y^{\prime}, c\right)\left(u\left(y^{\prime}\right)-c\right)^{2} d y^{\prime} \\
& -\int_{y_{c}}^{u^{-1}(z)} \frac{f\left(y^{\prime}, c\right)}{u^{\prime}\left(y^{\prime}\right)} \partial_{y}\left(\left(u\left(y^{\prime}\right)-c\right)^{2}\right) d y^{\prime}
\end{aligned}
$$


LINEAR INVISCID DAMPING FOR A CLASS OF MONOTONE SHEAR FLOW IN SOBOLEV SPACES 19

$$
=\int_{y_{c}}^{u^{-1}(z)} \partial_{c} f\left(y^{\prime}, c\right)\left(u\left(y^{\prime}\right)-c\right)^{2} d y^{\prime}+\int_{y_{c}}^{u^{-1}(z)} \partial_{y}\left(\frac{f\left(y^{\prime}, c\right)}{u^{\prime}\left(y^{\prime}\right)}\right)\left(u\left(y^{\prime}\right)-c\right)^{2} d y^{\prime} .
$$

With the above equalities, we can deduce that

$$
\begin{aligned}
& \left(\partial_{z}+\partial_{c}\right)^{2} \phi_{1}\left(u^{-1}(z), c\right) \\
& =2 \alpha^{2} \int_{y_{c}}^{u^{-1}(z)} \partial_{y}\left(\frac{1}{u^{\prime}\left(y^{\prime}\right)}\right) T_{2,2}\left(\partial_{c} \phi_{1}\right)\left(y^{\prime}, c\right) d y^{\prime}+\alpha^{2} \int_{y_{c}}^{u^{-1}(z)} T_{2,2}\left(\partial_{c}^{2} \phi_{1}\right)\left(y^{\prime}, c\right) d y^{\prime} \\
& \quad+\alpha^{2} \int_{y_{c}}^{u^{-1}(z)} T_{2,2} \phi_{1}\left(y^{\prime}, c\right)\left(\partial_{y}\left(\frac{1}{u^{\prime}\left(y^{\prime}\right)}\right)\right)^{2} d y^{\prime}+\alpha^{2} \int_{y_{c}}^{u^{-1}(z)} T_{2,2} \phi_{1}\left(y^{\prime}, c\right) \frac{1}{u^{\prime}\left(y^{\prime}\right)} \partial_{y}^{2}\left(\frac{1}{u^{\prime}\left(y^{\prime}\right)}\right) d y^{\prime} \\
& \quad+2 \alpha^{2} \int_{y_{c}}^{u^{-1}(z)} \partial_{y}\left(\frac{1}{u^{\prime}\left(y^{\prime}\right)}\right) T_{2,2}\left(\partial_{y}\left(\frac{\phi_{1}}{u^{\prime}}\right)\right)\left(y^{\prime}, c\right) d y^{\prime}+2 \alpha^{2} \int_{y_{c}}^{u^{-1}(z)} T_{2,2}\left(\partial_{c} \partial_{y}\left(\frac{\phi_{1}}{u^{\prime}}\right)\right)\left(y^{\prime}, c\right) d y^{\prime} \\
& \quad+\alpha^{2} \int_{y_{c}}^{u^{-1}(z)} T_{2,2}\left(\partial_{y}\left(\frac{1}{u^{\prime}} \partial_{y}\left(\frac{\phi_{1}}{u^{\prime}}\right)\right)\right)\left(y^{\prime}, c\right) d y^{\prime} .
\end{aligned}
$$

Note that

$$
\begin{aligned}
& \partial_{c}^{2} \phi_{1}+\partial_{y}\left(\frac{1}{u^{\prime}} \partial_{y}\left(\frac{\phi_{1}}{u^{\prime}}\right)\right)+2 \partial_{c} \partial_{y}\left(\frac{\phi_{1}}{u^{\prime}}\right) \\
& =\left(\partial_{z}+\partial_{c}\right)^{2} \phi_{1}\left(u^{-1}(z), c\right)+2 \partial_{y}\left(\frac{1}{u^{\prime}(y)}\right)\left(\partial_{z}+\partial_{c}\right) \phi_{1}\left(u^{-1}(z), c\right) \\
& \quad+\partial_{y}^{2}\left(\frac{1}{u^{\prime}(y)}\right) \frac{\phi_{1}}{u^{\prime}(y)}+\left(\partial_{y}\left(\frac{1}{u^{\prime}(y)}\right)\right)^{2} \phi_{1} .
\end{aligned}
$$

Thus, we obtain

$$
\begin{aligned}
\left(\partial_{z}\right. & \left.+\partial_{c}\right)^{2} \phi_{1}\left(u^{-1}(z), c\right) \\
= & \alpha^{2} T\left(\left(\partial_{z}+\partial_{c}\right)^{2} \phi_{1}\left(u^{-1}(z), c\right)\right)\left(u^{-1}(z), c\right)+2 \alpha^{2} T_{0}\left(\partial_{y}\left(\frac{1}{u^{\prime}\left(y^{\prime}\right)}\right) T_{2,2}\left(\left(\partial_{z}+\partial_{c}\right) \phi_{1}\left(u^{-1}(z), c\right)\right)\right) \\
& +\alpha^{2} \int_{y_{c}}^{u^{-1}(z)} T_{2,2} \phi_{1}\left(y^{\prime}, c\right)\left(\partial_{y}\left(\frac{1}{u^{\prime}\left(y^{\prime}\right)}\right)\right)^{2} d y^{\prime}+\alpha^{2} \int_{y_{c}}^{u^{-1}(z)} T_{2,2} \phi_{1}(y, c) \frac{1}{u^{\prime}(y)} \partial_{y}^{2}\left(\frac{1}{u^{\prime}(y)}\right) d y^{\prime} \\
& +2 \alpha^{2} \int_{y_{c}}^{u^{-1}(z)} \partial_{y}\left(\frac{1}{u^{\prime}\left(y^{\prime}\right)}\right) T_{2,2}\left(\phi_{1} \partial_{y}\left(\frac{1}{u^{\prime}}\right)\right) d y^{\prime}+2 \alpha^{2} T\left(\partial_{y}\left(\frac{1}{u^{\prime}}\right)\left(\partial_{z}+\partial_{c}\right) \phi_{1}\left(u^{-1}(z), c\right)\right) \\
& +\alpha^{2} T\left[\partial_{y}^{2}\left(\frac{1}{u^{\prime}}\right) \frac{\phi_{1}}{u^{\prime}}+\left(\partial_{y}\left(\frac{1}{u^{\prime}}\right)\right)^{2} \phi_{1}\right] .
\end{aligned}
$$

Then by using the estimate

$$
\left|\left(\partial_{y}+\partial_{c}\right) \phi_{1}\left(u^{-1}(y), c\right)\right| \leq C\left|\left(I-\alpha^{2} T\right)^{-1} \alpha^{2} T \phi_{1}\right|,
$$

and the fact that $T$ is a positive operator, we deduce that

$$
\begin{aligned}
& \left|\left(\partial_{y}+\partial_{c}\right)^{2} \phi_{1}\left(u^{-1}(y), c\right)\right| \\
& \leq C\left|\left(I-\alpha^{2} T\right)^{-1} \alpha^{2} T \phi_{1}\right|+C\left|\left(I-\alpha^{2} T\right)^{-1} \alpha^{2} T\left(I-\alpha^{2} T\right)^{-1} \alpha^{2} T \phi_{1}\right| \\
& \leq C\left|\alpha^{2} T\left(I-\alpha^{2} T\right)^{-2} 1\right|+C\left|\left(\alpha^{2} T\right)^{2}\left(I-\alpha^{2} T\right)^{-3} 1\right| \\
& \leq C \alpha\left(y-y_{c}\right) \sinh \alpha\left(y-y_{c}\right)+C\left|\sum_{k=0}^{\infty}(k+1)(k+2)\left(\alpha^{2} T\right)^{k+2} 1\right|
\end{aligned}
$$




$$
\leq C\left(\alpha\left(y-y_{c}\right) \sinh \alpha\left(y-y_{c}\right)+\alpha^{2}\left(y-y_{c}\right)^{2} \cosh \alpha\left(y-y_{c}\right)\right) .
$$

This deduces the second inequality.

\section{The inhomogeneous Rayleigh equation}

In this section, we consider the inhomogeneous Rayleigh equation

$$
\left\{\begin{array}{l}
\Phi^{\prime \prime}-\alpha^{2} \Phi-\frac{u^{\prime \prime}}{u-c} \Phi=f \\
\Phi(0)=\Phi(1)=0
\end{array}\right.
$$

Let $\phi(y, c)$ be the solution of the homogeneous Rayleigh equation given by Proposition 4.5, and $\phi_{1}(y, c) \triangleq \frac{\phi(y, c)}{u(y)-c}$. We denote

$$
\begin{aligned}
& \mathrm{II}_{2} \triangleq \text { p.v. } \int_{0}^{1} \frac{u^{\prime}(y)-u^{\prime}\left(y_{c}\right)}{(u(y)-c)^{2}} d y \\
& \mathrm{II}_{3} \triangleq \int_{0}^{1} \frac{1}{(u(y)-c)^{2}}\left(\frac{1}{\phi_{1}(y, c)^{2}}-1\right) d y
\end{aligned}
$$

for $c \in D_{0}$ and $y_{c}=u^{-1}(c)$.

The following lemma gives a sufficient and necessary condition so that $\mathcal{R}_{\alpha}$ has no embedding eigenvalues.

Lemma 6.1. Let $\mathrm{A}(c)=u(0)-u(1)-\rho(c) \mathrm{II}_{2}+u^{\prime}\left(y_{c}\right) \rho(c) \mathrm{II}_{3}$ and $\mathrm{B}(c)=\pi \rho(c) \frac{u^{\prime \prime}\left(y_{c}\right)}{u^{\prime}\left(y_{c}\right)^{2}}$, where $\rho(c)=(c-u(0))(u(1)-c)$. Then $c \in D_{0}$ is an embedding eigenvalue of $\mathcal{R}_{\alpha}$ if and only if

$$
\mathrm{A}(c)^{2}+\mathrm{B}(c)^{2}=0 .
$$

Proof. Let $c=u\left(y_{c}\right)$ be an embedding eigenvalue of $\mathcal{R}_{\alpha}$. We know that $u^{\prime \prime}\left(u^{-1}(c)\right)=0$, thus $\mathrm{B}(c)=0$. Next we show $\mathrm{A}(c)=0$. In such case, $\mathrm{A}(c)=0$ is equivalent to the Wronskian $W\left[\varphi_{1}, \varphi_{2} ; c\right]=0$, where $\varphi_{1}(y, c)$ and $\varphi_{2}(y, c)$ are two linearly independent solutions of the Rayleigh equation

$$
\varphi^{\prime \prime}-\alpha^{2} \varphi-\frac{u^{\prime \prime}}{u-c} \varphi=0 .
$$

Indeed, thanks to $u^{\prime \prime}\left(y_{c}\right)=0$, we can construct a smooth solution $\varphi(y, c)$ of $(6.4)$ with the boundary conditions $\varphi(0, c)=0$ and $\varphi^{\prime}(0, c)=1$. Moreover, we have

$$
W\left[\varphi_{1}, \varphi_{2} ; c\right]=\varphi(1, c)
$$

Let $\phi(y, c)$ be a solution of (6.4) given by Lemma 4.9 and $\phi_{1}(y, c)=\frac{\phi(y, c)}{u(y)-c}$. Then $\varphi(y, c)$ has the following representation formula

$$
\varphi(y, c)=\phi_{1}(0, c) \bar{\varphi}(y, c)
$$

where $\bar{\varphi}(y, c)$ is given by

$$
\begin{aligned}
\bar{\varphi}(y, c)= & \frac{\phi_{1}(y, c)}{u^{\prime}\left(y_{c}\right)}(u(y)-u(0)) \\
& +\frac{\phi_{1}(y, c)}{u^{\prime}\left(y_{c}\right)}(u(y)-c)(u(0)-c) \int_{0}^{y} \frac{u^{\prime}\left(y_{c}\right)-u^{\prime}(z)}{(u(z)-c)^{2}} d z \\
& +\phi_{1}(y, c)(u(y)-c)(u(0)-c) \int_{0}^{y} \frac{1}{(u(z)-c)^{2}}\left(\frac{1}{\phi_{1}(z, c)^{2}}-1\right) d z .
\end{aligned}
$$


Then we find that $\varphi(1, c)=0$ is equivalent to

$$
\begin{aligned}
0= & \varphi(1, c)=\phi_{1}(0, c) \bar{\varphi}(y, c) \\
= & \frac{\phi_{1}(0, c) \phi_{1}(1, c)}{u^{\prime}\left(y_{c}\right)}(u(1)-u(0))-\frac{\phi_{1}(0, c) \phi_{1}(1, c)}{u^{\prime}\left(y_{c}\right)} \rho(c) \int_{0}^{1} \frac{u^{\prime}\left(y_{c}\right)-u^{\prime}(y)}{(u(y)-c)^{2}} d y \\
& -\phi_{1}(0, c) \phi_{1}(1, c) \rho(c) \int_{0}^{1} \frac{1}{(u(y)-c)^{2}}\left(\frac{1}{\phi_{1}(y, c)^{2}}-1\right) d y \\
= & -\frac{\phi_{1}(0, c) \phi_{1}(1, c)}{u^{\prime}\left(y_{c}\right)} \mathrm{A}(c) .
\end{aligned}
$$

It remains to show that if $\mathrm{A}(c)^{2}+\mathrm{B}(c)^{2}=0$, then $c$ must be an embedding eigenvalue of $\mathcal{R}_{\alpha}$. The equality $\mathrm{A}(c)^{2}+\mathrm{B}(c)^{2}=0$ implies that $u^{\prime \prime}\left(y_{c}\right)=0$ and $\varphi(1, c)=0$. Then $\varphi(y, c)$ is a non zero solution of (6.4) with boundary conditions $\varphi(0, c)=\varphi(1, c)=0$. This means that $c$ is an embedding eigenvalue of $\mathcal{R}_{\alpha}$ with eigenfunction $\varphi(y, c)$.

Remark 6.2. For $c \in \mathbb{C} \backslash D_{0}$, let $\varphi(y, c)$ be the solution of (6.4) with $\varphi(0, c)=0$ and $\varphi^{\prime}(0, c)=1$, then $\varphi(y, c)$ is analytic in $c$, and $\lim _{c \rightarrow \infty} \varphi(y, c)=\frac{\sinh (\alpha y)}{\alpha}$. For $c \in \Omega_{\epsilon_{0}} \backslash D_{0}$, we have

$$
\varphi(y, c)=\phi(0, c) \phi(y, c) \int_{0}^{y} \frac{1}{\phi\left(y^{\prime}, c\right)^{2}} d y^{\prime}
$$

in particular,

$$
\varphi(1, c)=\phi(0, c) \phi(1, c) \int_{0}^{1} \frac{1}{\phi(y, c)^{2}} d y .
$$

Lemma 6.3. If $\mathcal{R}_{\alpha}$ has no embedding eigenvalues, then there exists $\epsilon_{1}>0$ such that for any $c \in \Omega_{\epsilon_{1}} \backslash D_{0}, \varphi(1, c) \neq 0$.

Proof. Let $c_{r}$ be defined by (4.3). We claim the following uniform convergence:

1. for $c_{\epsilon} \in\{\operatorname{Im} c>0\} \cap D_{\epsilon_{0}}$,

$$
\rho\left(c_{\epsilon}\right) \int_{0}^{1} \frac{1}{\phi\left(y, c_{\epsilon}\right)^{2}} d y \longrightarrow \frac{1}{u^{\prime}\left(y_{c}\right)}\left(\mathrm{A}\left(c_{r}\right)-i \mathrm{~B}\left(c_{r}\right)\right) \quad \text { as } c_{\epsilon} \rightarrow c_{r} ;
$$

2. for $c_{\epsilon} \in\{\operatorname{Im} c<0\} \cap D_{\epsilon_{0}}$,

$$
\rho\left(c_{\epsilon}\right) \int_{0}^{1} \frac{1}{\phi\left(y, c_{\epsilon}\right)^{2}} d y \longrightarrow \frac{1}{u^{\prime}\left(y_{c}\right)}\left(\mathrm{A}\left(c_{r}\right)+i \mathrm{~B}\left(c_{r}\right)\right) \quad \text { as } c_{\epsilon} \rightarrow c_{r} ;
$$

3. for $c_{\epsilon} \in B_{\epsilon_{0}}^{l} \cup B_{\epsilon_{0}}^{r}$,

$$
\rho\left(c_{\epsilon}\right) \int_{0}^{1} \frac{1}{\phi\left(y, c_{\epsilon}\right)^{2}} d y \longrightarrow \frac{u(0)-u(1)}{u^{\prime}\left(y_{c}\right)} \quad \text { as } c_{\epsilon} \rightarrow c_{r} .
$$

By definition of $\rho(c)$ and $\phi_{1}(y, c)$, we have

$$
\varphi(1, c)=-\rho(c) \phi_{1}(0, c) \phi_{1}(1, c) \int_{0}^{1} \frac{1}{\phi(y, c)^{2}} d y \quad \text { for } c \in \Omega_{\epsilon_{0}} \backslash D_{0} .
$$

It is easy to show that $A(c)$ and $B(c)$ are continuous in $D_{0}$. This along with the above claims implies that $\varphi(1, c)$ is continuous in $\Omega_{\epsilon_{0}}$. Thus, the conclusion follows from Lemma 6.1. 
Now let us first prove claim 1. Let $c_{\epsilon}=c+i \epsilon$ with $\epsilon \neq 0$ and $c \in D_{0}$ and $y_{c}=u^{-1}(c)$. We write

$$
\begin{aligned}
& \rho\left(c_{\epsilon}\right) \int_{0}^{1} \frac{1}{\phi\left(y, c_{\epsilon}\right)^{2}} d y \\
& =\rho\left(c_{\epsilon}\right) \int_{0}^{1} \frac{d y}{\left(u(y)-c_{\epsilon}\right)^{2} \phi_{1}\left(y, c_{\epsilon}\right)^{2}} \\
& =\rho\left(c_{\epsilon}\right) \int_{0}^{1} \frac{d y}{\left(u(y)-c_{\epsilon}\right)^{2}}+\rho\left(c_{\epsilon}\right) \int_{0}^{1} \frac{1}{\left(u(y)-c_{\epsilon}\right)^{2}}\left(\frac{1}{\phi_{1}\left(y, c_{\epsilon}\right)^{2}}-1\right) d y .
\end{aligned}
$$

By Lebesgue dominated convergence theorem, we get

$$
\lim _{\epsilon \rightarrow 0} \int_{0}^{1} \frac{1}{\left(u(y)-c_{\epsilon}\right)^{2}}\left(\frac{1}{\phi_{1}\left(y, c_{\epsilon}\right)^{2}}-1\right) d y=\int_{0}^{1} \frac{1}{(u(y)-c)^{2}}\left(\frac{1}{\phi_{1}(y, c)^{2}}-1\right) d y=\mathrm{II}_{3} .
$$

Thanks to $u^{\prime}\left(y_{c}\right)>c>0$, we have

$$
\begin{aligned}
& \rho\left(c_{\epsilon}\right) \int_{0}^{1} \frac{d y}{\left(u(y)-c_{\epsilon}\right)^{2}} \\
& =\frac{1}{u^{\prime}\left(y_{c}\right)} \rho\left(c_{\epsilon}\right)\left(\int_{0}^{1} \frac{u^{\prime}(y) d y}{\left(u(y)-c_{\epsilon}\right)^{2}}-\int_{0}^{1} \frac{u^{\prime}(y)-u^{\prime}\left(y_{c}\right)}{\left(u(y)-c_{\epsilon}\right)^{2}} d y\right) \\
& \triangleq \frac{1}{u^{\prime}\left(y_{c}\right)}\left(\mathrm{I}_{1}-\mathrm{I}_{2}\right) .
\end{aligned}
$$

Thanks to $\left|\frac{1}{\left(u(y)-c_{\epsilon}\right)^{2}}\right|>c \epsilon^{2}>0$, we get

$$
\begin{aligned}
\mathrm{I}_{1}=-\left.\frac{\left(c_{\epsilon}-u(0)\right)\left(u(1)-c_{\epsilon}\right)}{u(y)-c_{\epsilon}}\right|_{0} ^{1} & =\frac{\left(c_{\epsilon}-u(0)\right)\left(u(1)-c_{\epsilon}\right)}{u(0)-c_{\epsilon}}-\frac{\left(c_{\epsilon}-u(0)\right)\left(u(1)-c_{\epsilon}\right)}{u(1)-c_{\epsilon}} \\
& =u(0)-u(1) .
\end{aligned}
$$

Set $g(y)=u^{\prime}(y)-u^{\prime}\left(y_{c}\right)-\frac{u^{\prime \prime}\left(y_{c}\right)}{u^{\prime}\left(y_{c}\right)^{2}} u^{\prime}(y)(u(y)-c)$. Then $g\left(y_{c}\right)=0$ and $g^{\prime}\left(y_{c}\right)=0$.

$$
\begin{aligned}
\mathrm{I}_{2} & =\rho\left(c_{\epsilon}\right) \int_{0}^{1} \frac{u^{\prime}(y)-u^{\prime}\left(y_{c}\right)}{\left(u(y)-c_{\epsilon}\right)^{2}} d y \\
& =\rho\left(c_{\epsilon}\right) \int_{0}^{1} \frac{g(y)}{\left(u(y)-c_{\epsilon}\right)^{2}} d y+\rho\left(c_{\epsilon}\right) \frac{u^{\prime \prime}\left(y_{c}\right)}{u^{\prime}\left(y_{c}\right)^{2}} \int_{0}^{1} \frac{u^{\prime}(y)(u(y)-c)}{\left(u(y)-c_{\epsilon}\right)^{2}} d y .
\end{aligned}
$$

Because of $\left|g^{\prime}(y)\right|=\left|g^{\prime}(y)-g^{\prime}\left(y_{c}\right)\right| \leq C\left|y-y_{c}\right|$, we get

$$
\int_{0}^{1} \frac{g(y)}{\left(u(y)-c_{\epsilon}\right)^{2}} d y \rightarrow p . v \cdot \int_{0}^{1} \frac{g(y)}{(u(y)-c)^{2}} d y .
$$

Thus, we deduce that as $\epsilon \rightarrow 0+$,

$$
\begin{aligned}
& \rho\left(c_{\epsilon}\right) \int_{0}^{1} \frac{u^{\prime}(y)(u(y)-c)}{\left(u(y)-c_{\epsilon}\right)^{2}} d y=\rho\left(c_{\epsilon}\right) \int_{u(0)-c_{\epsilon}}^{u(1)-c_{\epsilon}} \frac{x+i \epsilon}{x^{2}} d x \\
& =\rho\left(c_{\epsilon}\right) \ln \frac{u(1)-c_{\epsilon}}{u(0)-c_{\epsilon}}+i \epsilon(u(0)-u(1)) \\
& \longrightarrow(c-u(0))(u(1)-c) \ln \frac{u(1)-c}{c-u(0)}+i \pi(c-u(0))(u(1)-c) \\
& =\text { p.v. } \rho(c) \int_{0}^{1} \frac{u^{\prime}(y)(u(y)-c)}{(u(y)-c)^{2}} d y+i \pi \rho(c) .
\end{aligned}
$$


This shows that

$$
\mathrm{I}_{2} \longrightarrow \rho(c) \mathrm{II}_{2}+i \pi \frac{u^{\prime \prime}\left(y_{c}\right)}{u^{\prime}\left(y_{c}\right)^{2}} \rho(c) \quad \text { as } \epsilon \rightarrow 0+.
$$

So, we have as $\epsilon \rightarrow 0+$,

$$
\rho\left(c_{\epsilon}\right) \int_{0}^{1} \frac{1}{\phi\left(y, c_{\epsilon}\right)^{2}} d y \longrightarrow \frac{1}{u^{\prime}\left(y_{c}\right)}\left(u(0)-u(1)-\rho(c) \mathrm{II}_{2}-i \pi \frac{u^{\prime \prime}\left(y_{c}\right)}{u^{\prime}\left(y_{c}\right)^{2}} \rho(c)\right)+\rho(c) \mathrm{II}_{3} .
$$

This shows claim 1, and the proof of claim 2 is similar.

Let us prove claim 3. Let $c_{\epsilon}=u(0)+\epsilon e^{i \theta}$ with $\epsilon>0$. Similarly, we write

$$
\begin{aligned}
& \rho\left(c_{\epsilon}\right) \int_{0}^{1} \frac{1}{\phi\left(y, c_{\epsilon}\right)^{2}} d y \\
& =\rho\left(c_{\epsilon}\right) \int_{0}^{1} \frac{d y}{\left(u(y)-c_{\epsilon}\right)^{2}}+\rho\left(c_{\epsilon}\right) \int_{0}^{1} \frac{1}{\left(u(y)-c_{\epsilon}\right)^{2}}\left(\frac{1}{\phi_{1}\left(y, c_{\epsilon}\right)^{2}}-1\right) d y .
\end{aligned}
$$

Because $\frac{1}{\left(u(y)-c_{\epsilon}\right)^{2}}\left(\frac{1}{\phi_{1}\left(y, c_{\epsilon}\right)^{2}}-1\right)$ is uniformly bounded, the second term tends to zero. For the first term, we write as above

$$
\rho\left(c_{\epsilon}\right) \int_{0}^{1} \frac{d y}{\left(u(y)-c_{\epsilon}\right)^{2}} \triangleq \frac{1}{u^{\prime}(0)}\left(\mathrm{I}_{1}-\mathrm{I}_{2}\right) .
$$

It's easy to see that

$$
\mathrm{I}_{1}=u(0)-u(1) .
$$

For $\mathrm{I}_{2}$, we have

$$
\begin{aligned}
\left|\mathrm{I}_{2}\right| & =\left|\epsilon e^{i \theta}\left(u(1)-u(0)-\epsilon e^{i \theta}\right) \int_{u(0)}^{u(1)} \frac{u^{\prime}\left(u^{-1}(z)\right)-u^{\prime}(0)}{\left(z-u(0)-\epsilon e^{i \theta}\right)^{2}}\left(u^{-1}\right)^{\prime}(z) d z\right| \\
& \leq C \epsilon \int_{0}^{u(1)-u(0)} \frac{z}{\sqrt{(z+\epsilon \cos \theta)^{2}+\epsilon^{2} \sin ^{2} \theta}} d z \\
& \leq C \epsilon|\ln \epsilon| \rightarrow 0 \quad \text { as } \epsilon \rightarrow 0 .
\end{aligned}
$$

For the case of $c_{\epsilon} \in B_{\epsilon_{0}}^{l}$, the proof is similar.

Remark 6.4. If $A(c)<0$ and $B(c)=0$ for $c \in D_{0}$, then $\mathcal{R}_{\alpha}$ has no eigenvalue.

Indeed, under this assumption we can find $0<\epsilon_{1}<1$ such that for any $c \in \overline{\Omega_{\epsilon_{1}}} \backslash D_{0}$, $\varphi(1, c) \notin(-\infty, 0]$. Since $\varphi(1, c)$ is analytic for $c \in \mathbb{C} \backslash D_{0}$, and $\varphi(1, c) \rightarrow \frac{\sinh \alpha}{\alpha}$ for $c \rightarrow \infty$, we can find $R>2$ such that $\varphi(1, c) \in \mathbb{C} \backslash(-\infty, 0]$ for $|c| \geq R$. Then by residue theorem, the number of roots of $\varphi(1, c)$ for $c \in \mathbb{C} \backslash D_{0}$ equals to the number of roots of $\varphi(1, c)$ for $c \in B_{R} \backslash \overline{\Omega_{\epsilon_{1}}}$, which equals to(count multipilcity)

$$
\frac{1}{2 \pi i} \oint_{\partial B_{R}}-\oint_{\partial \Omega_{\epsilon_{1}}} \frac{\partial_{c} \varphi(1, c)}{\varphi(1, c)} d c=\frac{1}{2 \pi i} \oint_{\partial B_{R}}-\oint_{\partial \Omega_{\epsilon_{1}}} \partial_{c} \ln \varphi(1, c) d c .
$$

Let $z=\varphi(1, c)$. For $c \in \partial B_{R}, \ln z$ is the analytic function defined in $\mathbb{C} \backslash(-\infty, 0]$ such that $\ln z=\ln |z|+i \arg z$ for $|z|>0,-\pi<\arg z<\pi$. For $c \in \partial \Omega_{\epsilon_{1}}$, by our assumption, if we take $\epsilon_{1}$ small enough, such that $\ln (z)=\ln |z|+i \arg z$ for $|z|>0,-\pi<\arg z<\pi$. Thus, we deduce that

$$
\frac{1}{2 \pi i} \oint_{\partial B_{R}}-\oint_{\partial \Omega_{\epsilon_{1}}} \partial_{c} \ln \varphi(1, c) d c=0
$$


Proposition 6.5. Let $c \in \Omega_{\epsilon_{0}}$ with $\epsilon_{1}$ as in Lemma 6.3. Suppose that $\mathcal{R}_{\alpha}$ has no embedding eigenvalues. Then we have the following representation formula for the solution of (6.1):

$$
\Phi(y, c)=\phi(y, c) \int_{0}^{y} \frac{1}{\phi(z, c)^{2}} \int_{y_{c}}^{z} \phi\left(y^{\prime}, c\right) f\left(y^{\prime}, c\right) d y^{\prime} d z+\mu(c) \phi(y, c) \int_{0}^{y} \frac{1}{\phi\left(y^{\prime}, c\right)^{2}} d y^{\prime},
$$

where $y_{c}=u^{-1}\left(c_{r}\right)$ with $c_{r}$ defined by (4.3) and

$$
\mu(c)=-\frac{\int_{0}^{1} \frac{1}{\phi(z, c)^{2}} \int_{y_{c}}^{z} \phi f\left(y^{\prime}, c\right) d y^{\prime} d z}{\int_{0}^{1} \frac{1}{\phi(y, c)^{2}} d y} .
$$

Proof. Let $\varphi(y)$ be a solution of the homogenous Rayleigh equation

$$
\varphi^{\prime \prime}-\alpha^{2} \varphi-\frac{u^{\prime \prime}}{u-c} \varphi=0,
$$

then the inhomogeneous Rayleigh equation

$$
\left\{\begin{array}{l}
\Phi^{\prime \prime}-\alpha^{2} \Phi-\frac{u^{\prime \prime}}{u-c} \Phi=f \\
\Phi(0)=\Phi(1)=0
\end{array}\right.
$$

is equivalent to

$$
\left\{\begin{array}{l}
\left(\varphi^{2}\left(\frac{\Phi}{\varphi}\right)^{\prime}\right)^{\prime}=f \varphi \\
\psi(0)=\psi(1)=0
\end{array}\right.
$$

This gives our result by integration and noting that $\mu(c)$ is well-defined by Lemma 6.3.

Next we study the convergence of the solution $\psi(y, c)$ with $f=\frac{\omega_{0}(y)}{i \alpha(u-c)}$. Let $c \in D_{0}$ and $y_{c}=u^{-1}(c)$. We introduce

$$
\Phi_{ \pm}(y, c) \triangleq \begin{cases}\phi \int_{0}^{y} \frac{1}{\phi(z, c)^{2}} \int_{y_{c}}^{z} \phi f\left(y^{\prime}, c\right) d y^{\prime} d z+\mu_{ \pm}(c) \phi \int_{0}^{y} \frac{1}{\phi\left(y^{\prime}, c\right)^{2}} d y^{\prime} & 0 \leq y \leq y_{c} \\ \phi \int_{1}^{y} \frac{1}{\phi(z, c)^{2}} \int_{y_{c}}^{z} \phi f\left(y^{\prime}, c\right) d y^{\prime} d z+\mu_{ \pm}(c) \phi \int_{1}^{y} \frac{1}{\phi\left(y^{\prime}, c\right)^{2}} d y^{\prime} & y_{c} \leq y \leq 1\end{cases}
$$

and

$$
\begin{aligned}
& \Phi_{l}(y) \triangleq \phi(y, u(0)) \int_{1}^{y} \frac{1}{\phi(z, u(0))^{2}} \int_{0}^{z} \phi\left(y^{\prime}, u(0)\right) f\left(y^{\prime}, u(0)\right) d y^{\prime} d z \\
& \Phi_{r}(y) \triangleq \phi(y, u(1)) \int_{0}^{y} \frac{1}{\phi(z, u(1))^{2}} \int_{1}^{z} \phi\left(y^{\prime}, u(1)\right) f\left(y^{\prime}, u(1)\right) d y^{\prime} d z
\end{aligned}
$$

where

$$
\begin{aligned}
& \mu_{+}(c)=\frac{1}{\alpha} \frac{i u^{\prime}\left(y_{c}\right) \rho(c) \mathrm{II}_{1}-\rho(c) \frac{\omega_{0}\left(y_{c}\right)}{u^{\prime}\left(y_{c}\right)} \pi}{u(0)-u(1)-\rho(c) \mathrm{II}_{2}-i \pi \rho(c) \frac{u^{\prime \prime}\left(y_{c}\right)}{u^{\prime}\left(y_{c}\right)^{2}}+u^{\prime}\left(y_{c}\right) \rho(c) \mathrm{II}_{3}}, \\
& \mu_{-}(c)=\frac{1}{\alpha} \frac{i u^{\prime}\left(y_{c}\right) \rho(c) \mathrm{II}_{1}+\rho(c) \frac{\omega_{0}\left(y_{c}\right)}{u^{\prime}\left(y_{c}\right)} \pi}{u(0)-u(1)-\rho(c) \mathrm{II}_{2}+i \pi \rho(c) \frac{u^{\prime \prime}\left(y_{c}\right)}{u^{\prime}\left(y_{c}\right)^{2}}+u^{\prime}\left(y_{c}\right) \rho(c) \mathrm{II}_{3}},
\end{aligned}
$$

with

$$
\mathrm{II}_{1}\left(\omega_{0}\right)=\mathrm{p} \cdot \mathrm{v} \cdot \int_{0}^{1} \frac{\int_{y_{c}}^{z} \omega_{0}\left(y^{\prime}\right) \phi_{1}\left(y^{\prime}, c\right) d y^{\prime}}{\phi(z, c)^{2}} d z
$$


Lemma 6.6. Suppose that $f(y, c)$ is a Lipschitz function in $y \in[0,1]$ with

$$
|f(y, c)-f(z, c)| \leq C|y-z| \quad \text { for }(y, z, c) \in[0,1]^{2} \times D_{\epsilon_{0}},
$$

and $f\left(y, c_{\epsilon}\right)$ uniformly converges to $f(y, c)$ for $c_{\epsilon}=c+i \epsilon, c \in D_{0}$ as $\epsilon \rightarrow 0$. Then it holds that for $0 \leq y \leq y_{c}$,

$$
\left(y-y_{c}+i \epsilon\right) \int_{0}^{y} \frac{f\left(z, c_{\epsilon}\right)}{\left(z-y_{c}+i \epsilon\right)^{2}} d z \longrightarrow\left(y-y_{c}\right) \int_{0}^{y} \frac{f(z, c)}{\left(z-y_{c}\right)^{2}} d z
$$

as $\epsilon$ tends to zero.

Proof. Thanks to $0 \leq z \leq y \leq y_{c}$, then $\left|z-y_{c}\right| \geq\left|y-y_{c}\right|$ and

$$
\left|\frac{\left(f\left(z, c_{\epsilon}\right)-f\left(y_{c}, c_{\epsilon}\right)\right)\left(y-y_{c}+i \epsilon\right)}{\left(z-y_{c}+i \epsilon\right)^{2}}\right| \leq C \frac{\left|z-y_{c}\right|\left(\left|y-z_{0}\right|^{2}+\epsilon^{2}\right)^{1 / 2}}{\left|z-y_{c}\right|^{2}+\epsilon^{2}} \leq C,
$$

which together with Lebesgue dominated convergence theorem gives

$$
\left(y-y_{c}+i \epsilon\right) \int_{0}^{y} \frac{f\left(z, c_{\epsilon}\right)-f\left(y_{c}, c_{\epsilon}\right)}{\left(z-y_{c}+i \epsilon\right)^{2}} d z \longrightarrow\left(y-y_{c}\right) \int_{0}^{y} \frac{f(z, c)-f\left(y_{c}, c\right)}{\left(z-y_{c}\right)^{2}} d z .
$$

On the other hand, we have

$$
\begin{aligned}
\left(y-y_{c}+i \epsilon\right) \int_{0}^{y} \frac{1}{\left(y-y_{c}+i \epsilon\right)^{2}} d z & =-\frac{y-y_{c}+i \epsilon}{-y_{c}+i \epsilon}+1 \\
& \longrightarrow\left(y-y_{c}\right) \int_{0}^{y} \frac{1}{\left(z-y_{c}\right)^{2}} d z
\end{aligned}
$$

as $\epsilon$ tends to zero. Then the lemma follows easily.

Proposition 6.7. Let $\Phi(y, c)$ be a solution of (6.1) given by Proposition 6.5 with $f=\frac{\omega_{0}(y)}{i \alpha(u-c)}$ for $\omega_{0} \in L^{2}(0,1)$. If $\mathcal{R}_{\alpha}$ has no embedding eigenvalues, then it holds that

1. for any $\left(y, y_{c}\right) \in[0,1] \times[0,1]$,

$$
\lim _{\epsilon \rightarrow 0+} \Phi\left(y, c_{\epsilon}\right)=\Phi_{+}\left(y, u\left(y_{c}\right)\right), \quad \lim _{\epsilon \rightarrow 0-} \Phi\left(y, c_{\epsilon}\right)=\Phi_{-}\left(x, u\left(y_{c}\right)\right),
$$

where $c_{\epsilon}=c+i \epsilon$ and $c=u\left(y_{c}\right)$.

2. for any $y \in[0,1]$,

$$
\lim _{\epsilon \rightarrow 0+} \Phi\left(y, c_{\epsilon}^{l}\right)=\Phi_{l}(y), \quad \lim _{\epsilon \rightarrow 0+} \Phi\left(y, c_{\epsilon}^{r}\right)=\Phi_{r}(y),
$$

where $c_{\epsilon}^{l}=u(0)+\epsilon e^{i \theta}$ and $c_{\epsilon}^{r}=u(1)-\epsilon e^{i \theta}$ for $\theta \in\left[\frac{\pi}{2}, \frac{3 \pi}{2}\right]$.

Proof. Let us first prove the first statement. To show the convergence of $\mu\left(c_{\epsilon}\right)$, we consider

$$
\begin{aligned}
\mu\left(c_{\epsilon}\right) & =-\frac{\int_{0}^{1} \frac{1}{\phi\left(z, c_{\epsilon}\right)^{2}} \int_{y_{c}}^{z} \phi f\left(y, c_{\epsilon}\right) d y d z}{\int_{0}^{1} \frac{1}{\phi\left(y, c_{\epsilon}\right)^{2}} d y} \\
& =-\frac{\rho\left(c_{\epsilon}\right) \int_{0}^{1} \frac{1}{\phi\left(z, c_{\epsilon}\right)^{2}} \int_{y_{c}}^{z} \phi f\left(y, c_{\epsilon}\right) d y d z}{\rho\left(c_{\epsilon}\right) \int_{0}^{1} \frac{1}{\phi\left(y, c_{\epsilon}\right)^{2}} d y} .
\end{aligned}
$$

By the claims in the proof of Lemma 6.3, it suffices to deal with the numerator of $\mu(c)$, which is decomposed as

$$
\rho\left(c_{\epsilon}\right) \int_{0}^{1} \frac{1}{\phi\left(z, c_{\epsilon}\right)^{2}} \int_{y_{c}}^{z} \phi f\left(y, c_{\epsilon}\right) d y d z=\rho\left(c_{\epsilon}\right) \int_{0}^{1} \frac{1}{\phi\left(z, c_{\epsilon}\right)^{2}} \int_{y_{c}}^{z} \phi_{1}\left(y, c_{\epsilon}\right) \frac{\omega_{0}(y)}{i \alpha} d y d z
$$




$$
\begin{aligned}
= & \rho\left(c_{\epsilon}\right) \int_{0}^{1} \frac{1}{\phi\left(z, c_{\epsilon}\right)^{2}} \int_{y_{c}}^{z}\left(\phi_{1}\left(y, c_{\epsilon}\right)-1\right) \frac{\omega_{0}(y)}{i \alpha} d y d z \\
& +\rho\left(c_{\epsilon}\right) \int_{0}^{1} \frac{\int_{y_{c}}^{y} \frac{\omega_{0}(y)}{i \alpha} d y}{\left(u(z)-c_{\epsilon}\right)^{2}}\left(\frac{1}{\phi_{1}\left(z, c_{\epsilon}\right)^{2}}-1\right) d z \\
& +\rho\left(c_{\epsilon}\right) \int_{0}^{1} \frac{\int_{y_{c}}^{z} \frac{\omega_{0}(y)}{i \alpha} d y}{\left(u(z)-c_{\epsilon}\right)^{2}} d z .
\end{aligned}
$$

Using the inequality $\left|\phi_{1}\left(y, c_{\epsilon}\right)-1\right| \leq C\left(\left|y-y_{c}\right|^{2}+\epsilon^{2}\right)$ and Lebesgue dominated convergence theorem, we get

$$
\begin{aligned}
& \rho\left(c_{\epsilon}\right) \int_{0}^{1} \frac{1}{\phi\left(z, c_{\epsilon}\right)^{2}} \int_{y_{c}}^{z}\left(\phi_{1}\left(y, c_{\epsilon}\right)-1\right) \frac{\omega_{0}(y)}{i \alpha} d y d z \\
& \longrightarrow \rho(c) \int_{0}^{1} \frac{1}{\phi(z, c)^{2}} \int_{y_{c}}^{z}\left(\phi_{1}(y, c)-1\right) \frac{\omega_{0}(y)}{i \alpha} d y d z
\end{aligned}
$$

and

$$
\rho\left(c_{\epsilon}\right) \int_{0}^{1} \frac{\int_{y_{c}}^{y} \frac{\omega_{0}(y)}{i \alpha} d y}{\left(u(z)-c_{\epsilon}\right)^{2}}\left(\frac{1}{\phi_{1}\left(z, c_{\epsilon}\right)^{2}}-1\right) d z \longrightarrow \rho(c) \int_{0}^{1} \frac{\int_{y_{c}}^{z} \frac{\omega_{0}(y)}{i \alpha} d y}{(u(z)-c)^{2}}\left(\frac{1}{\phi_{1}(z, c)^{2}}-1\right) d z
$$

as $\epsilon$ tends to zero. Similar to the proof of $\mathrm{I}_{2}$ in Lemma 6.3, we have

$$
\rho\left(c_{\epsilon}\right) \int_{0}^{1} \frac{\int_{x_{0}}^{z} \frac{\omega_{0}(y)}{i \alpha} d y}{\left(u(z)-c_{\epsilon}\right)^{2}} d z \rightarrow \rho(c) \int_{0}^{1} \frac{\int_{x_{0}}^{z} \frac{\omega_{0}(y)}{i \alpha} d y}{(u(z)-c)^{2}} d z+\rho(c) \frac{\omega_{0}\left(y_{c}\right)}{i \alpha u^{\prime}\left(y_{c}\right)^{2}} \pi i .
$$

as $\varepsilon \rightarrow 0+$. Thus, we obtain as $\epsilon \rightarrow 0+$,

$$
\begin{aligned}
& \rho\left(c_{\epsilon}\right) \int_{0}^{1} \frac{1}{\phi\left(z, c_{\epsilon}\right)^{2}} \int_{y_{c}}^{z} \phi f\left(y, c_{\epsilon}\right) d y d z \\
& \longrightarrow \text { p.v. } \rho(c) \int_{0}^{1} \frac{\int_{y_{c}}^{z} \frac{\omega_{0}(y) \phi_{1}(y, c)}{i \alpha} d y}{\phi(z, c)^{2}} d z+\rho(c) \frac{\omega_{0}\left(y_{c}\right)}{i \alpha u^{\prime}\left(y_{c}\right)^{2}} \pi i \\
& =-\frac{1}{\alpha}\left(i \rho(c) \mathrm{II}_{1}\left(\omega_{0}\right)-\rho(c) \frac{\omega_{0}\left(y_{c}\right)}{u^{\prime}\left(y_{c}\right)^{2}} \pi\right) .
\end{aligned}
$$

Similarly, we have that $\epsilon \rightarrow 0-$,

$$
\rho\left(c_{\epsilon}\right) \int_{0}^{1} \frac{1}{\phi\left(z, c_{\epsilon}\right)^{2}} \int_{y_{c}}^{z} \phi f\left(y, c_{\epsilon}\right) d y d z \longrightarrow-\frac{1}{\alpha}\left(i \rho(c) \mathrm{II}_{1}+\rho(c) \frac{\omega_{0}\left(y_{c}\right)}{u^{\prime}\left(y_{c}\right)^{2}} \pi\right) .
$$

This shows by Lemma 6.1 that

$$
\mu\left(c_{\epsilon}\right) \longrightarrow \mu_{ \pm}(c) \text { as } \epsilon \rightarrow 0 \pm .
$$

Next we show the convergence of $\psi\left(y, c_{\epsilon}\right)$. Using the formula

$$
\begin{aligned}
\phi\left(y, c_{\epsilon}\right) & =\left(u(y)-u\left(y_{c}\right)-i \epsilon\right) \phi_{1}\left(y, c_{\epsilon}\right) \\
& =\left(\left(y-y_{c}\right) \int_{0}^{1} u^{\prime}\left(y_{c}+t\left(y-y_{c}\right)\right) d t-i \epsilon\right) \phi_{1}\left(y, c_{\epsilon}\right),
\end{aligned}
$$

we deduce from Lemma 6.6 that as $\epsilon \rightarrow 0$,

$$
\phi\left(y, c_{\epsilon}\right) \int_{0}^{y} \frac{1}{\phi\left(z, c_{\epsilon}\right)^{2}} d y \longrightarrow \phi(y, c) \int_{0}^{y} \frac{1}{\phi(y, c)^{2}} d y
$$


LINEAR INVISCID DAMPING FOR A CLASS OF MONOTONE SHEAR FLOW IN SOBOLEV SPACES 27

$$
\phi\left(y, c_{\epsilon}\right) \int_{0}^{y} \frac{1}{\phi\left(z, c_{\epsilon}\right)^{2}} \int_{y_{c}}^{z} \phi f\left(y^{\prime}, c_{\epsilon}\right) d y^{\prime} d z \longrightarrow \phi(y, c) \int_{0}^{y} \frac{1}{\phi(z, c)^{2}} \int_{y_{c}}^{z} \phi f\left(y^{\prime}, c\right) d y^{\prime} d z,
$$

for $y_{c} \geq y \geq 0$, and

$$
\begin{aligned}
& \phi\left(y, c_{\epsilon}\right) \int_{1}^{y} \frac{1}{\phi\left(y, c_{\epsilon}\right)^{2}} d y \longrightarrow \phi(y, c) \int_{1}^{y} \frac{1}{\phi\left(y^{\prime}, c\right)^{2}} d y^{\prime} \\
& \phi\left(y, c_{\epsilon}\right) \int_{1}^{y} \frac{1}{\phi\left(z, c_{\epsilon}\right)^{2}} \int_{y_{c}}^{z} \phi f\left(y^{\prime}, c_{\epsilon}\right) d y d z \longrightarrow \phi \int_{1}^{x} \frac{1}{\phi(z, c)^{2}} \int_{y_{c}}^{z} \phi f\left(y^{\prime}, c\right) d y^{\prime} d z,
\end{aligned}
$$

for $y_{c} \leq y \leq 1$. This finishes the proof of the first statement.

The second statement is similar just by noting that in this case, we have

$$
\mu\left(c_{\epsilon}^{l}\right) \longrightarrow 0, \mu\left(c_{\epsilon}^{r}\right) \longrightarrow 0
$$

as $\epsilon \rightarrow 0+$.

\section{Representation FORMula of the SOlution}

Let $\widehat{\psi}(t, \alpha, y)$ be the solution of the linearized Euler equations

$$
\frac{1}{i \alpha} \partial_{t} \widehat{\psi}=\left(\partial_{y}^{2}-\alpha^{2}\right)^{-1}\left(u^{\prime \prime}(y)-u(y)\left(\partial_{y}^{2}-\alpha^{2}\right)\right) \widehat{\psi}=-\mathcal{R}_{\alpha} \widehat{\psi}
$$

with the initial data

$$
\psi(0, \alpha, y)=\left(\alpha^{2}-\partial_{y}^{2}\right)^{-1} \widehat{\omega}_{0}(\alpha, y)
$$

where $\widehat{\omega}_{0}(\alpha, y)=\mathcal{F}_{x} \omega_{0}$. We know from $(3.5)$ that

$$
\widehat{\psi}(t, \alpha, y)=\frac{1}{2 \pi i} \int_{\partial \Omega} e^{-i \alpha t c}\left(c-\mathcal{R}_{\alpha}\right)^{-1} \widehat{\psi}(0, \alpha, y) d c,
$$

where $\Omega$ is a simple connected domain including the spectrum $\sigma\left(\mathcal{R}_{\alpha}\right)$ of $\mathcal{R}_{\alpha}$.

Note that $P_{i \alpha \mathcal{R}_{\alpha}} \widehat{\psi}(0, \alpha, y)=0$ if $P_{\mathcal{L}} \omega_{0}=0$. Thus, we have

$$
\widehat{\psi}(t, \alpha, y)=\frac{1}{2 \pi} \int_{\partial \Omega_{\epsilon}} e^{-i \alpha t c}\left(c-\mathcal{R}_{\alpha}\right)^{-1} \widehat{\psi}(0, \alpha, y) d c,
$$

where $\Omega_{\epsilon}$ defined by (4.2) with $\epsilon$ sufficiently small.

Let $\Phi(\alpha, y, c)$ be the solution of $(6.1)$ with $f(\alpha, y, c)=\frac{\widehat{\omega}_{0}(\alpha, y)}{i \alpha(u-c)}$. It is easy to see that

$$
\left(c-\mathcal{R}_{\alpha}\right)^{-1} \widehat{\psi}(0, \alpha, y)=i \alpha \Phi(\alpha, y, c) .
$$

This gives

$$
\widehat{\psi}(t, \alpha, y)=\frac{1}{2 \pi} \int_{\partial \Omega_{\epsilon}} \alpha \Phi(\alpha, y, c) e^{-i \alpha c t} d c .
$$

Since $\mathcal{L}\left(\right.$ thus, $\mathcal{R}_{\alpha}$ ) has no embedding eigenvalues, Lemma 6.3 ensures that there are no eigenvalues of $\mathcal{R}_{\alpha}$ in $\Omega_{\epsilon}$ for $\epsilon$ small enough. Then it follows from Proposition 6.7 that

$$
\begin{aligned}
\widehat{\psi}(t, \alpha, y) & =\frac{1}{2 \pi} \int_{\partial \Omega_{\epsilon}} \alpha \Phi(\alpha, y, c) e^{-i \alpha c t} d c \\
& =\lim _{\epsilon \rightarrow 0^{+}} \frac{1}{2 \pi} \int_{\partial \Omega_{\epsilon}} \alpha \Phi(\alpha, y, c) e^{-i \alpha c t} d c \\
& =\frac{1}{2 \pi} \int_{u(0)}^{u(1)} \alpha \widetilde{\Phi}(y, c) e^{-i \alpha c t} d c,
\end{aligned}
$$


where

$$
\widetilde{\Phi}(y, c)= \begin{cases}\left(\mu_{-}(c)-\mu_{+}(c)\right) \phi(y, c) \int_{0}^{y} \frac{1}{\phi(z, c)^{2}} d z & 0 \leq y<y_{c}, \\ \left(\mu_{-}(c)-\mu_{+}(c)\right) \phi(y, c) \int_{1}^{y} \frac{1}{\phi(z, c)^{2}} d z & y_{c}<y \leq 1\end{cases}
$$

with $y_{c}=u(c)$, and $\phi(y, c)$ is the solution of (4.1) given by Proposition 4.5.

We denote

$$
\begin{aligned}
& \mathrm{A} \triangleq u(0)-u(1)-\rho(c) \mathrm{II}_{2}+u^{\prime}\left(y_{c}\right) \rho(c) \mathrm{II}_{3}, \\
& \mathrm{~B} \triangleq \pi \rho(c) \frac{u^{\prime \prime}\left(y_{c}\right)}{u^{\prime}\left(y_{c}\right)^{2}}, \\
& \mathrm{C} \triangleq \rho(c) \frac{\widehat{\omega}_{0}\left(\alpha, y_{c}\right)}{u^{\prime}\left(y_{c}\right)} \pi, \quad \mathrm{D} \triangleq u^{\prime}\left(y_{c}\right) \rho(c) \mathrm{II}_{1}\left(\widehat{\omega}_{0}\right),
\end{aligned}
$$

where $\rho(c)=(c-u(0))(u(1)-c)$, and $\mathrm{II}_{1}, \mathrm{II}_{2}$ and $\mathrm{II}_{3}$ are given by (6.5), (6.2) and (6.3) respectively. Then we have

$$
\mu_{-}(c)-\mu_{+}(c)=\frac{2}{\alpha} \frac{\mathrm{AC}+\mathrm{BD}}{\mathrm{A}^{2}+\mathrm{B}^{2}} \triangleq \frac{2}{\alpha} \rho(c) \mu(c) .
$$

\section{Sobolev Regularity of $\mu(c)$}

In this section, we study the regularity of $\mu(c)$ defined by (7.3). The result is stated as follows.

Proposition 8.1. With the same assumptions as in Theorem 1.1, there exists a constant $C$ independent of $\alpha$ such that

1. $L^{2}$ estimates

$$
\begin{aligned}
& \|\rho \mu\|_{L^{2}} \leq \frac{C}{\alpha}\left\|\widehat{\omega}_{0}(\alpha, \cdot)\right\|_{L^{2}} \\
& \|\mu\|_{L^{2}} \leq C\left\|\widehat{\omega}_{0}(\alpha, \cdot)\right\|_{L^{2}} .
\end{aligned}
$$

2. $W^{1,2}$ estimates

$$
\begin{aligned}
& \left\|\partial_{c}(\rho \mu)\right\|_{L^{2}} \leq C\left\|\widehat{\omega}_{0}(\alpha, \cdot)\right\|_{H^{1}}, \\
& \left\|\partial_{c} \mu\right\|_{L^{2}} \leq C(1+\alpha)\left\|\widehat{\omega}_{0}(\alpha, \cdot)\right\|_{H^{1}} .
\end{aligned}
$$

3. $W^{2,2}$ estimates

$$
\begin{aligned}
& \left\|\partial_{c}^{2}(\rho \mu)\right\|_{L^{2}} \leq C(1+\alpha)\left\|\widehat{\omega}_{0}(\alpha, \cdot)\right\|_{H^{2}}, \\
& \left\|\rho \partial_{c}^{2}(\rho \mu)\right\|_{L^{2}} \leq C\left\|\widehat{\omega}_{0}(\alpha, \cdot)\right\|_{H^{2}} .
\end{aligned}
$$

Proof. Let us first estimate $\mathrm{II}_{1}, \mathrm{II}_{2}$ and $\mathrm{II}_{3}$. Recall that

$$
\begin{aligned}
& \mathrm{II}_{1}\left(\widehat{\omega}_{0}\right)=\text { p.v. } \int_{0}^{1} \frac{\int_{y_{c}}^{z} \widehat{\omega}_{0}\left(\alpha, y^{\prime}\right) \phi_{1}\left(y^{\prime}, c\right) d y^{\prime}}{\phi(z, c)^{2}} d z, \\
& \mathrm{II}_{2}=\text { p.v. } \int_{0}^{1} \frac{u^{\prime}(y)-u^{\prime}\left(y_{c}\right)}{(u(y)-c)^{2}} d y, \\
& \mathrm{II}_{3}=\int_{0}^{1} \frac{1}{(u(y)-c)^{2}}\left(\frac{1}{\phi_{1}(y, c)^{2}}-1\right) d y .
\end{aligned}
$$


LINEAR INVISCID DAMPING FOR A CLASS OF MONOTONE SHEAR FLOW IN SOBOLEV SPACES 29

By a change of variable, $\mathrm{II}_{1}$ can be written as

$$
\operatorname{II}_{1}\left(\widehat{\omega}_{0}\right)(c)=\mathcal{L}_{1}\left(\widehat{\omega}_{0}\left(\alpha, u^{-1}\right)\left(u^{-1}\right)^{\prime}\right) .
$$

Then Lemma 11.4, Lemma11.9 and Lemma11.10 ensure that

$$
\begin{aligned}
& \left\|\operatorname{II}_{1}\left(\widehat{\omega}_{0}\right)\right\|_{L^{p}} \leq C\left\|\widehat{\omega}_{0}(\alpha, \cdot)\right\|_{L^{p}}, \\
& \left\|\partial_{c}\left(\rho \mathrm{II}_{1}\left(\widehat{\omega}_{0}\right)\right)\right\|_{L^{p}} \leq C\left\|\widehat{\omega}_{0}(\alpha, \cdot)\right\|_{W^{1, p}}, \\
& \left\|\partial_{c}^{2}\left(\rho^{2} \mathrm{II}_{1}\left(\widehat{\omega}_{0}\right)\right)\right\|_{L^{p}} \leq C\left\|\widehat{\omega}_{0}(\alpha, \cdot)\right\|_{W^{2, p}} .
\end{aligned}
$$

Let $y_{c}=u^{-1}(c)$ in the sequel. We rewrite $\mathrm{II}_{2}$ as

$$
\begin{aligned}
\mathrm{II}_{2}(c)= & \mathrm{p} \cdot \mathrm{v} \cdot \int_{0}^{1} \frac{\int_{y_{c}}^{z} u^{\prime \prime}(y) d y}{(u(z)-c)^{2}} d z \\
= & -\frac{1}{u^{\prime}\left(y_{c}\right)} \int_{0}^{1} \frac{\left(\int_{0}^{1} u^{\prime \prime}\left(z+t\left(z-y_{c}\right)\right) d t\right)^{2}}{\left(\int_{0}^{1} u^{\prime}\left(z+t\left(z-y_{c}\right)\right) d t\right)^{2}} d z \\
& +\frac{1}{u^{\prime}\left(y_{c}\right)} \mathrm{p} \cdot \mathrm{v} \cdot \int_{0}^{1} \frac{-u^{\prime}(z) \int_{y_{c}}^{z} u^{\prime \prime}(y) d y}{(u(z)-c)^{2}} d z \\
\triangleq & \mathrm{II}_{2,1}+\frac{1}{u^{\prime}\left(y_{c}\right)} \mathrm{II}_{2,2} .
\end{aligned}
$$

It's easy to see that

$$
\left\|\mathrm{II}_{2,1}\right\|_{L^{\infty}}+\left\|\partial_{c} \mathrm{II}_{2,1}\right\|_{L^{\infty}}+\left\|\partial_{c}^{2} \mathrm{II}_{2,1}\right\|_{L^{\infty}} \leq C
$$

We rewrite $\mathrm{II}_{2,2}$ as

$$
\begin{aligned}
\mathrm{II}_{2,2}(c)= & \int_{u(0)}^{u(1)} \frac{\int_{c}^{c^{\prime}} u^{\prime \prime}\left(u^{-1}(z)\right)\left(u^{-1}\right)^{\prime}(z) d z}{\left(c^{\prime}-c\right)^{2}} d c^{\prime} \\
= & -\chi_{D_{0}}(c) H\left(u^{\prime \prime}\left(u^{-1}\right)\left(u^{-1}\right)^{\prime} \chi_{D_{0}}\right)(c) \\
& +\frac{1}{c-u(0)} \int_{u(0)}^{c} u^{\prime \prime}\left(u^{-1}(z)\right)\left(u^{-1}\right)^{\prime}(z) d z-\frac{1}{u(1)-c} \int_{c}^{u(0)} u^{\prime \prime}\left(u^{-1}(z)\right)\left(u^{-1}\right)^{\prime}(z) d z,
\end{aligned}
$$

from which and Lemma 11.5, it follows that

$$
\left\|\mathrm{II}_{2,2}\right\|_{L^{p}}+\left\|\partial_{c}\left(\rho \mathrm{II}_{2,2}\right)\right\|_{L^{p}}+\left\|\partial_{c}^{2}\left(\rho^{2} \mathrm{II}_{2,2}\right)\right\|_{L^{p}} \leq C .
$$

Thus, we deduce that for any $p \in(1, \infty)$,

$$
\begin{aligned}
& \left\|\mathrm{II}_{2}\right\|_{L^{p}}+\left\|\partial_{c}\left(\rho \mathrm{II}_{2}\right)\right\|_{L^{p}}+\left\|\partial_{c}^{2}\left(\rho^{2} \mathrm{II}_{2}\right)\right\|_{L^{p}} \leq C, \\
& \left\|\rho \mathrm{II}_{2}\right\|_{L^{\infty}}+\left\|\partial_{c}\left(\rho^{2} \mathrm{II}_{2}\right)\right\|_{L^{\infty}} \leq C .
\end{aligned}
$$

We get by Lemma 5.4 that

$$
\begin{aligned}
\left|\mathrm{II}_{3}(c)\right| & =\int_{0}^{1} \frac{1}{(u(y)-c)^{2}}\left(1-\frac{1}{\phi_{1}(y, c)^{2}}\right) d y \\
& \geq C^{-1} \int_{0}^{1} \frac{1}{\left(y-y_{c}\right)^{2}}\left(\frac{\phi_{1}^{2}(y, c)-1}{\phi_{1}(y, c)^{2}}\right) d y \\
& \geq C^{-1} \alpha \int_{-\alpha y_{c}}^{\alpha\left(1-y_{c}\right)} \frac{1}{\sinh ^{2} y}\left(\frac{\sinh y}{y}-1\right) d y \\
& \geq C^{-1} \alpha \int_{0}^{1 / 2} \frac{1}{\sinh ^{2} y}\left(\frac{\sinh y}{y}-1\right) d y \geq C^{-1} \alpha
\end{aligned}
$$


and

$$
\begin{aligned}
\left|\mathrm{II}_{3}(c)\right| & =\int_{0}^{1} \frac{1}{(u(y)-c)^{2}}\left(1-\frac{1}{\phi_{1}(y, c)^{2}}\right) d y \\
& \leq C \alpha \int_{-\alpha y_{c}}^{\alpha\left(1-y_{c}\right)} \frac{1}{y \sinh y}\left(\frac{\sinh y}{y}-1\right) d y \\
& \leq C \alpha\left(1+\int_{|y|>1} \frac{1}{y^{2}}+\frac{1}{y \sinh y} d y\right) \leq C \alpha .
\end{aligned}
$$

This gives

$$
C^{-1} \alpha \leq\left|\mathrm{II}_{3}(c)\right| \leq C \alpha
$$

By Remark 5.5, we have

$$
\begin{aligned}
\partial_{c} \mathrm{II}_{3}(c)= & \partial_{c} \int_{0}^{1} \frac{1}{(u(y)-c)^{2}}\left(\frac{1}{\phi_{1}(y, c)^{2}}-1\right) d y \\
= & \int_{0}^{1}-\partial_{y}\left(\frac{1}{(u(y)-c)^{2}}\right) \frac{1}{u^{\prime}(y)}\left(\frac{1}{\phi_{1}(y, c)^{2}}-1\right) d y \\
& +\int_{0}^{1} \frac{1}{(u(y)-c)^{2}} \partial_{c}\left(\frac{1}{\phi_{1}(y, c)^{2}}-1\right) d y \\
= & \left.\frac{\alpha^{2} \mathcal{T}\left(\phi_{1}\right)(y, c)\left(\phi_{1}(y, c)+1\right)}{\phi_{1}(y, c)^{2} u^{\prime}\left(y_{c}\right) \int_{0}^{1} u^{\prime}\left(y_{c}+t\left(y-y_{c}\right)\right) d t}\right|_{y=0} ^{1} \\
& +\int_{0}^{1} \frac{1}{(u(y)-c)^{2}}\left(\partial_{c}+\frac{1}{u^{\prime}(y)} \partial_{y}\right)\left(\frac{1}{\phi_{1}(y, c)^{2}}\right) d y \\
& +\int_{0}^{1} \frac{1}{(u(y)-c)^{2}}\left(\frac{1}{u^{\prime}(y)}\right)^{\prime}\left(\frac{1}{\phi_{1}(y, c)^{2}}-1\right) d y
\end{aligned}
$$

and

$$
\begin{aligned}
\partial_{c}^{2} \mathrm{II}_{3}(c)= & \partial_{c}\left(\left.\frac{\alpha^{2} \mathcal{T}\left(\phi_{1}\right)(y, c)\left(\phi_{1}(y, c)+1\right)}{\phi_{1}(y, c)^{2} u^{\prime}\left(y_{c}\right) \int_{0}^{1} u^{\prime}\left(y_{c}+t\left(y-y_{c}\right)\right) d t}\right|_{y=0} ^{1}\right) \\
& +\int_{0}^{1}-\partial_{y}\left(\frac{1}{(u(y)-c)^{2}}\right) \frac{1}{u^{\prime}(y)}\left(\partial_{c}+\frac{1}{u^{\prime}(y)} \partial_{y}\right)\left(\frac{1}{\phi_{1}(y, c)^{2}}\right) d y \\
& +\int_{0}^{1}-\partial_{y}\left(\frac{1}{(u(y)-c)^{2}}\right) \frac{1}{u^{\prime}(y)}\left(\frac{1}{u^{\prime}(y)}\right)^{\prime}\left(\frac{1}{\phi_{1}(y, c)^{2}}-1\right) d y \\
& +\int_{0}^{1}\left(\frac{1}{(u(y)-c)^{2}}\right) \partial_{c}\left(\partial_{c}+\frac{1}{u^{\prime}(y)} \partial_{y}\right)\left(\frac{1}{\phi_{1}(y, c)^{2}}\right) d y \\
& +\int_{0}^{1} \frac{1}{(u(y)-c)^{2}}\left(\frac{1}{u^{\prime}(y)}\right)^{\prime} \partial_{c}\left(\frac{1}{\phi_{1}(y, c)^{2}}-1\right) d y \\
= & \partial_{c}\left(\left.\frac{\alpha^{2} \mathcal{T}\left(\phi_{1}\right)(y, c)\left(\phi_{1}(y, c)+1\right)}{\phi_{1}(y, c)^{2} u^{\prime}\left(y_{c}\right) \int_{0}^{1} u^{\prime}\left(y_{c}+t\left(y-y_{c}\right)\right) d t}\right|_{y=0} ^{1}\right) \\
& -\left.\frac{1}{(u(y)-c)^{2}} \frac{1}{u^{\prime}(y)}\left(\partial_{c}+\frac{1}{u^{\prime}(y)} \partial_{y}\right)\left(\frac{1}{\phi_{1}(y, c)^{2}}\right)\right|_{y=0} ^{1} \\
& -\left.\frac{1}{(u(y)-c)^{2}} \frac{1}{u^{\prime}(y)}\left(\frac{1}{u^{\prime}(y)}\right)^{\prime}\left(\frac{1}{\phi_{1}(y, c)^{2}}-1\right)\right|_{y=0} ^{1}
\end{aligned}
$$


LINEAR INVISCID DAMPING FOR A CLASS OF MONOTONE SHEAR FLOW IN SOBOLEV SPACES 31

$$
\begin{aligned}
& +\int_{0}^{1} \frac{1}{(u(y)-c)^{2}}\left(\frac{1}{u^{\prime}(y)}\right)^{\prime}\left(\partial_{c}+\frac{1}{u^{\prime}(y)} \partial_{y}\right)\left(\frac{1}{\phi_{1}(y, c)^{2}}\right) d y \\
& +\int_{0}^{1} \frac{1}{(u(y)-c)^{2}}\left(\partial_{c}+\frac{1}{u^{\prime}(y)} \partial_{y}\right)^{2}\left(\frac{1}{\phi_{1}(y, c)^{2}}\right) d y \\
& +\int_{0}^{1} \frac{1}{(u(y)-c)^{2}}\left(\frac{1}{u^{\prime}(y)}\left(\frac{1}{u^{\prime}(y)}\right)^{\prime}\right)^{\prime}\left(\frac{1}{\phi_{1}(y, c)^{2}}-1\right) d y \\
& +\int_{0}^{1} \frac{1}{(u(y)-c)^{2}}\left(\frac{1}{u^{\prime}(y)}\right)^{\prime}\left(\frac{1}{u^{\prime}(y)} \partial_{y}+\partial_{c}\right)\left(\frac{1}{\phi_{1}(y, c)^{2}}\right) d y
\end{aligned}
$$

Then by Remark 5.5 again, Proposition 5.1 and Proposition 5.6, we obtain

$$
\left\|\partial_{c} \mathrm{II}_{3}\right\|_{L^{\infty}} \leq C \alpha^{2}, \quad\left\|\partial_{c}^{2} \mathrm{II}_{3}\right\|_{L^{\infty}} \leq C \alpha^{3}, \quad\left\|\partial_{c}\left(\rho^{2} \partial_{c} \mathrm{II}_{3}\right)\right\|_{L^{\infty}} \leq C \alpha .
$$

Now let us turn to the estimate of $\mu(c)$.

$$
\begin{aligned}
\mu(c)= & \frac{\pi \rho(c) \mathrm{II}_{3}(c) \widehat{\omega}_{0}\left(\alpha, y_{c}\right)}{\mathrm{A}^{2}+\mathrm{B}^{2}}-\frac{\pi \rho(c) \mathrm{II}_{2}(c) \widehat{\omega}_{0}\left(\alpha, y_{c}\right)}{u^{\prime}\left(y_{c}\right)\left(\mathrm{A}^{2}+\mathrm{B}^{2}\right)} \\
& +\frac{\pi(u(0)-u(1)) \widehat{\omega}_{0}\left(\alpha, y_{c}\right)}{u^{\prime}\left(y_{c}\right)\left(\mathrm{A}^{2}+\mathrm{B}^{2}\right)}+\frac{\pi u^{\prime \prime}\left(y_{c}\right) \rho \mathrm{II}_{1}\left(\widehat{\omega}_{0}\right)(c)}{u^{\prime}\left(y_{c}\right)\left(\mathrm{A}^{2}+\mathrm{B}^{2}\right)} .
\end{aligned}
$$

By Lemma $6.1, \mathrm{~A}^{2}+\mathrm{B}^{2} \geq C^{-1}$. Thus, by (8.6), we get

$$
\mathrm{A}^{2}+\mathrm{B}^{2} \geq C^{-1}\left((1+\alpha \rho)^{2}+\rho^{2}\right) .
$$

Thus, by (8.1), (8.6) and (8.5), we get

$$
\|\mu\|_{L^{2}} \leq C\left(1+\frac{1}{\alpha}\left\|\mathrm{II}_{3}\right\|_{L^{\infty}}+\left\|\rho \mathrm{II}_{2}\right\|_{L^{\infty}}\right)\left\|\widehat{\omega}_{0}(\alpha, \cdot)\right\|_{L^{2}}+C\left\|\mathrm{II}_{1}\right\|_{L^{2}} \leq C\left\|\widehat{\omega}_{0}(\alpha, \cdot)\right\|_{L^{2}} .
$$

Using the inequality $(1+\alpha \rho)^{2}+\rho^{2} \geq C^{-1}(\rho \alpha)^{k}$ for $k=1,2$, we can deduce that

$$
\|\rho \mu\|_{L^{2}} \leq \frac{C}{\alpha}\left\|\widehat{\omega}_{0}(\alpha, \cdot)\right\|_{L^{2}}
$$

The $H^{1}$ estimate of $\mu(c)$ is similar. Here we just show the estimate of one term.

$$
\partial_{c}\left(\frac{\rho \mathrm{II}_{1}\left(\widehat{\omega}_{0}\right)(c)}{\mathrm{A}^{2}+\mathrm{B}^{2}}\right)=\frac{\partial_{c}\left(\rho \mathrm{II}_{1}\left(\widehat{\omega}_{0}\right)(c)\right)}{\mathrm{A}^{2}+\mathrm{B}^{2}}-\frac{\rho \mathrm{II}_{1}\left(\widehat{\omega}_{0}\right)(c) \partial_{c}\left(\mathrm{~A}^{2}+\mathrm{B}^{2}\right)}{\left(\mathrm{A}^{2}+\mathrm{B}^{2}\right)^{2}} .
$$

We infer from (8.2) and (8.6) that

$$
\left\|\frac{\partial_{c}\left(\rho \mathrm{II}_{1}\left(\widehat{\omega}_{0}\right)(c)\right)}{\mathrm{A}^{2}+\mathrm{B}^{2}}\right\|_{L^{2}} \leq C\left\|\widehat{\omega}_{0}(\alpha, \cdot)\right\|_{L^{2}} .
$$

Notice that

$$
\begin{aligned}
\left|\partial_{c}\left(\mathrm{~A}^{2}+\mathrm{B}^{2}\right)\right| & \leq\left|2 \mathrm{~A}\left(\partial_{c}\left(u^{\prime}\left(y_{c}\right) \rho(c) \mathrm{II}_{3}\right)-\partial_{c}\left(\rho(c) \mathrm{II}_{2}\right)\right)+2 \mathrm{~B} \partial_{c} \mathrm{~B}\right| \\
& \leq C\left(\mathrm{~A}^{2}+\mathrm{B}^{2}\right)^{1 / 2}\left(1+\left|\partial_{c}\left(\rho(c) \mathrm{II}_{3}\right)\right|+\left|\rho \mathrm{II}_{3}\right|+\left|\partial_{c}\left(\rho(c) \mathrm{II}_{2}\right)\right|\right)
\end{aligned}
$$

Thus, by (8.1), (8.7), (8.4) and Sobolev embedding, we get

$$
\begin{aligned}
& \left\|\frac{\rho \mathrm{II}_{1}\left(\widehat{\omega}_{0}\right)(c) \partial_{c}\left(\mathrm{~A}^{2}+\mathrm{B}^{2}\right)}{\left(\mathrm{A}^{2}+\mathrm{B}^{2}\right)^{2}}\right\|_{L^{2}} \\
& \leq C\left\|\frac{\partial_{c}\left(\rho(c) \mathrm{II}_{2}\right)}{\left(\mathrm{A}^{2}+\mathrm{B}^{2}\right)^{3 / 2}}\right\|_{L^{4}}\left\|\rho \mathrm{II}_{1}\left(\widehat{\omega}_{0}\right)\right\|_{L^{4}}+C\left(\frac{1}{\alpha}\left\|\partial_{c} \mathrm{II}_{3}\right\|_{L^{\infty}}+\left\|\mathrm{II}_{3}\right\|_{L^{\infty}}\right)\left\|\rho \mathrm{II}_{1}\left(\widehat{\omega}_{0}\right)\right\|_{L^{2}} \\
& \leq C \alpha\left\|\widehat{\omega}_{0}(\alpha, \cdot)\right\|_{H^{1}}
\end{aligned}
$$


which gives

$$
\left\|\partial_{c}\left(\frac{\rho \mathrm{II}_{1}\left(\widehat{\omega}_{0}\right)(c)}{\mathrm{A}^{2}+\mathrm{B}^{2}}\right)\right\|_{L^{2}} \leq C \alpha\left\|\widehat{\omega}_{0}(\alpha, \cdot)\right\|_{H^{1}} .
$$

The $H^{2}$ estimate of $\mu(c)$ is similar. We left it to the interested readers.

\section{Uniform Sobolev estimates of the vorticity}

Recall that $\widehat{\omega}(t, \alpha, y)$ satisfies

$$
\left\{\begin{array}{l}
\partial_{t} \widehat{\omega}+i \alpha u \widehat{\omega}+i \alpha u^{\prime \prime} \widehat{\psi}=0 \\
\left.\widehat{\omega}\right|_{t=0}=\mathcal{F} \omega_{0}(\alpha, y) .
\end{array}\right.
$$

This is equivalent to

$$
\left(e^{i \alpha t u(y)} \widehat{\omega}(t, \alpha, y)\right)_{t}=-i \alpha e^{i \alpha t u(y)} u^{\prime \prime}(y) \widehat{\psi}(t, \alpha, y) .
$$

Integration in $t$ gives

$$
e^{i \alpha t u(y)} \widehat{\omega}(t, \alpha, y)=\widehat{\omega}_{0}(\alpha, y)-i \alpha u^{\prime \prime}(y) \int_{0}^{t} e^{i \tau u(y)} \widehat{\psi}(\tau, \alpha, y) d \tau .
$$

We denote $W(t, x, y) \triangleq \omega(t, x+u(y) t, y)$. We find that

$$
\widehat{W}(t, \alpha, y)=e^{i \alpha t u(y)} \widehat{\omega}(t, \alpha, y) .
$$

Then we get by (7.2) that

$$
\begin{aligned}
\widehat{W}(t, \alpha, y) & =\widehat{\omega}_{0}(\alpha, y)-\frac{u^{\prime \prime}(y)}{\pi} \int_{u(0)}^{u(1)} \frac{\left(e^{i t(u(y)-c) \alpha}-1\right) \rho(c) \mu(c) \Gamma(y, c)}{u(y)-c} d c \\
& \triangleq \widehat{\omega}_{0}(\alpha, y)-\frac{u^{\prime \prime}(y)}{\pi} \mathbf{T}(\mu)(t, y),
\end{aligned}
$$

where

$$
\Gamma(y, c)= \begin{cases}\phi(y, c) \int_{0}^{y} \frac{1}{\phi(z, c)^{2}} d z \triangleq \Gamma_{0}(y, c) & 0 \leq y<y_{c} \\ \phi(y, c) \int_{1}^{y} \frac{1}{\phi(z, c)^{2}} d z \triangleq \Gamma_{1}(y, c) & y_{c}<y \leq 1 .\end{cases}
$$

We have the following uniform estimates in Sobolev space for $W(t, x, y)$.

Proposition 9.1. With the same assumptions as in Theorem 1.1, it holds that

$$
\begin{aligned}
& \|W(t)\|_{H_{x}^{-1} L_{y}^{2}} \leq C\left\|w_{0}\right\|_{H_{x}^{-1} L_{y}^{2}}, \\
& \|W(t)\|_{H_{x}^{-1} H_{y}^{1}} \leq C\left\|w_{0}\right\|_{H_{x}^{-1} H_{y}^{1}}, \\
& \left\|\rho(u(y)) \partial_{y}^{2} W(t)\right\|_{H_{x}^{-1} L_{y}^{2}} \leq C\left\|w_{0}\right\|_{H_{x}^{-1} H_{y}^{2}} .
\end{aligned}
$$

Proof. The proof is split into three steps.

Step 1. $L^{2}$ estimate

Let $\varphi(y) \in C_{0}^{\infty}(0,1)$ with $\|\varphi\|_{L^{2}}=1$. A direct calculation gives

$$
\int_{0}^{1} \mathbf{T}(\mu)(t, y) \varphi(y) d y
$$


LINEAR INVISCID DAMPING FOR A CLASS OF MONOTONE SHEAR FLOW IN SOBOLEV SPACES 33

$$
\begin{aligned}
= & \int_{0}^{1} \int_{u(0)}^{u(1)} \frac{\left(e^{i t(u(y)-c) \alpha}-1\right) \rho(c) \mu(c) \Gamma(y, c)}{u(y)-c} d c \varphi(y) d y \\
= & \int_{0}^{1} \int_{u(0)}^{u(y)} \frac{\left(e^{i t(u(y)-c) \alpha}-1\right) \rho(c) \mu(c) \Gamma(y, c)}{u(y)-c} d c \varphi(y) d y \\
& +\int_{0}^{1} \int_{u(y)}^{u(1)} \frac{\left(e^{i t(u(y)-c) \alpha}-1\right) \rho(c) \mu(c) \Gamma(y, c)}{u(y)-c} d c \varphi(y) d y \\
= & \int_{u(0)}^{u(1)} \rho(c) \mu(c) \int_{y_{c}}^{1} \frac{\left(e^{i t(u(y)-c) \alpha}-1\right) \Gamma_{1}(y, c) \varphi(y)}{u(y)-c} d y d c \\
& +\int_{u(0)}^{u(1)} \rho(c) \mu(c) \int_{0}^{y_{c}} \frac{\left(e^{i t(u(y)-c) \alpha}-1\right) \Gamma_{0}(y, c) \varphi(y)}{u(y)-c} d y d c \\
= & \int_{u(0)}^{u(1)} \rho(c) \mu(c) \int_{y_{c}}^{1}\left(e^{i t(u(y)-c) \alpha}-1\right) \phi_{1}(y, c) \int_{1}^{y} \frac{1}{\phi(z, c)^{2}} d z \varphi(y) d y d c \\
& +\int_{u(0)}^{u(1)} \rho(c) \mu(c) \int_{0}^{y_{c}}\left(e^{i t(u(y)-c) \alpha}-1\right) \phi_{1}(y, c) \int_{0}^{y} \frac{1}{\phi(z, c)^{2}} d z \varphi(y) d y d c \\
= & \int_{u(0)}^{u(1)} \rho(c) \mu(c) \int_{0}^{1} \frac{-1}{\phi(z, c)^{2}} \int_{y_{c}}^{z}\left(e^{i t(u(y)-c) \alpha}-1\right) \varphi(y) \phi_{1}(y, c) d y d z d c \\
= & -\int_{u(0)}^{u(1)} \rho(c) \mu(c) e^{-i \alpha c t} \mathbb{T}\left(\left(u^{-1}\right)^{\prime}, \varphi \circ u^{-1} e^{i t \alpha z}\left(u^{-1}\right)^{\prime}, \phi_{1}\left(u^{-1}, c\right), \frac{1}{\phi_{1}\left(u^{-1}, c\right)^{2}}\right)(c) d c \\
& +\int_{u(0)}^{u(1)} \rho(c) \mu(c) \mathbb{T}\left(\left(u^{-1}\right)^{\prime}, \varphi \circ u^{-1}\left(u^{-1}\right)^{\prime}, \phi_{1}\left(u^{-1}, c\right), \frac{1}{\phi_{1}\left(u^{-1}, c\right)^{2}}\right)(c) d c . \\
&
\end{aligned}
$$

Then by Lemma 11.7 and Proposition 8.1, we get

$$
\int_{0}^{1} \mathbf{T}(\mu)(t, y) \varphi(y) d y \leq C\|\rho(c) \mu(c)\|_{L^{2}}\|\varphi\|_{L^{2}} \leq C\left\|\widehat{\omega}_{0}(\alpha, \cdot)\right\|_{L^{2}} .
$$

This gives

$$
\|W(t)\|_{H_{x}^{-1} L_{y}^{2}} \leq C\left\|\omega_{0}\right\|_{H_{x}^{-1} L_{y}^{2}}
$$

Step 2. $H^{1}$ estimate

Let $\varphi(y) \in C_{0}^{\infty}(0,1)$ with $\|\varphi\|_{L^{2}}=1$. Then we have

$$
\begin{aligned}
& \int_{0}^{1} \partial_{y} \mathbf{T}(\rho \mu)(t, y) \varphi(y) d y=-\int_{0}^{1} \mathbf{T}(\rho \mu)(t, y) \varphi^{\prime}(y) d y \\
& =\int_{u(0)}^{u(1)} \rho(c) \mu(c) \int_{0}^{1} \frac{1}{\phi\left(y^{\prime}, c\right)^{2}} \int_{y_{c}}^{y^{\prime}}\left(e^{i t(u(y)-c) \alpha}-1\right) \varphi^{\prime}(y) \phi_{1}(y, c) d y d y^{\prime} d c \\
& =\int_{u(0)}^{u(1)} \rho(c) \mu(c) \mathbb{T}\left(\left(u^{-1}\right)^{\prime},\left(\varphi \circ u^{-1}\right)^{\prime}, e^{i t \alpha(z-c)} \phi_{1}\left(u^{-1}, c\right), \frac{1}{\phi_{1}\left(u^{-1}, c\right)^{2}}\right)(c) d c \\
& \quad-\int_{u(0)}^{u(1)} \rho(c) \mu(c) \mathbb{T}\left(\left(u^{-1}\right)^{\prime},\left(\varphi \circ u^{-1}\right)^{\prime}, \phi_{1}\left(u^{-1}, c\right), \frac{1}{\phi_{1}\left(u^{-1}, c\right)^{2}}\right)(c) d c .
\end{aligned}
$$


We get by Lemma 11.2 that

$$
\begin{aligned}
\int_{0}^{1} & \partial_{y} \mathbf{T}(\rho \mu(\cdot))(t, y) \varphi(y) d y \\
= & -\int_{u(0)}^{u(1)} \rho(c) \mu(c) \partial_{c} \mathcal{L}_{1}\left(\varphi \circ u^{-1}\right)(c) d c+\int_{u(0)}^{u(1)} \rho(c) \mu(c) \mathcal{L}_{2}\left(\varphi \circ u^{-1}\right)(c) d c \\
& +\int_{u(0)}^{u(1)} \rho(c) \mu(c) \partial_{c}\left(e^{-i t \alpha c} \mathcal{L}_{1}\left(\varphi \circ u^{-1} e^{i t \alpha z}\right)(c)\right) d c \\
& -\int_{u(0)}^{u(1)} \rho(c) \mu(c) e^{-i t \alpha c} \mathcal{L}_{2}\left(\varphi \circ u^{-1} e^{i t \alpha z}\right)(c) d c \\
& +\int_{u(0)}^{u(1)} \rho(c) \mu(c) e^{-i t \alpha c} \mathcal{B}\left(\varphi \circ u^{-1} e^{i t \alpha z}\right)(c) d c-\int_{u(0)}^{u(1)} \rho(c) \mu(c) \mathcal{B}\left(\varphi \circ u^{-1}\right)(c) d c \\
= & \int_{u(0)}^{u(1)} \partial_{c}(\rho(c) \mu(c)) \mathcal{L}_{1}\left(\varphi \circ u^{-1}\right)(c) d c+\int_{u(0)}^{u(1)} \rho(c) \mu(c) \mathcal{L}_{2}\left(\varphi \circ u^{-1}\right)(c) d c \\
& -\int_{u(0)}^{u(1)} \partial_{c}(\rho(c) \mu(c))\left(e^{-i t \alpha c} \mathcal{L}_{1}\left(\varphi \circ u^{-1}(z) e^{i t \alpha z}\right)(c)\right) d c \\
& -\int_{u(0)}^{u(1)} \rho(c) \mu(c) e^{-i t \alpha c} \mathcal{L}_{2}\left(\varphi \circ u^{-1} e^{i t \alpha z}\right)(c) d c \\
& +\int_{u(0)}^{u(1)} \rho(c) \mu(c) e^{-i t \alpha c} \mathcal{B}\left(\varphi \circ u^{-1}(z) e^{i t \alpha z}\right)(c) d c-\int_{u(0)}^{u(1)} \rho(c) \mu(c) \mathcal{B}\left(\varphi \circ u^{-1}\right)(c) d c .
\end{aligned}
$$

Thus, by Lemma 11.10, Lemma 11.9 and Proposition 8.1, we obtain

$$
\begin{aligned}
& \int_{0}^{1} \partial_{y} \mathbf{T}(\rho \mu(\cdot))(t, y) \varphi(y) d y \\
& \leq\left\|\partial_{c}(\rho(c) \mu(c))\right\|_{L^{2}}\left(\left\|\mathcal{L}_{1}\left(\varphi \circ u^{-1}\right)\right\|_{L^{2}}+\left\|\mathcal{L}_{1}\left(e^{i t \alpha z} \varphi \circ u^{-1}\right)\right\|_{L^{2}}\right) \\
& \quad+C\|\rho \mu\|_{L^{2}}\left(\left\|\mathcal{L}_{2}\left(\varphi \circ u^{-1}\right)\right\|_{L^{2}}+\left\|\mathcal{L}_{2}\left(e^{i t \alpha z} \varphi \circ u^{-1}\right)\right\|_{L^{2}}\right. \\
& \left.\quad+\left\|\mathcal{B}\left(\varphi \circ u^{-1}\right)\right\|_{L^{2}}+\left\|\mathcal{B}\left(e^{i t \alpha z} \varphi \circ u^{-1}\right)\right\|_{L^{2}}\right) \\
& \leq C\left\|\partial_{c}(\rho(c) \mu(c))\right\|_{L^{2}}+C\|\rho \mu\|_{L^{2}} \leq C\left\|\widehat{\omega_{0}}(\alpha, \cdot)\right\|_{H^{1}} .
\end{aligned}
$$

This gives

$$
\|W(t)\|_{H_{x}^{-1} H_{y}^{1}} \leq C\left\|\omega_{0}\right\|_{H_{x}^{-1} H_{y}^{1}}
$$

Step 3. $H^{2}$ estimate

Let $\varphi(y) \in C_{0}^{\infty}(0,1)$ with $\|\varphi\|_{L^{2}}=1$. Then we have

$$
\begin{aligned}
& \int_{0}^{1} \rho(u(y)) \partial_{y}^{2} \mathbf{T}(\rho \mu(\cdot))(t, y) \varphi(y) d y \\
& =\int_{0}^{1} \mathbf{T}(\rho \mu(\cdot))(t, y) \partial_{y}^{2}(\varphi(y) \rho(u(y))) d y \\
& =-\int_{u(0)}^{u(1)} \rho(c) \mu(c) \int_{0}^{1} \frac{1}{\phi\left(y^{\prime}, c\right)^{2}} \int_{y_{c}}^{y^{\prime}}\left(e^{i t(u(y)-c) \alpha}-1\right) \partial_{y}^{2}(\varphi(y) \rho(u(y))) \phi_{1}(y, c) d y d y^{\prime} d c,
\end{aligned}
$$


LINEAR INVISCID DAMPING FOR A CLASS OF MONOTONE SHEAR FLOW IN SOBOLEV SPACES 35 where

$$
\begin{aligned}
& \int_{0}^{1} \frac{1}{\phi^{2}(z, c)} \int_{x_{0}}^{z}\left(e^{i t(u(y)-c) \alpha}-1\right) \partial_{y}^{2}(\varphi(y) \rho(u(y))) \phi_{1}(y, c) d y d z \\
& =\mathbb{T}\left(\left(u^{-1}\right)^{\prime},\left(\partial_{y}^{2}(\varphi(y) \rho(u(y))) \circ u^{-1}\right)\left(u^{-1}\right)^{\prime},\left(e^{i t(z-c) \alpha}-1\right) \phi_{1}\left(u^{-1}(z), c\right), \frac{1}{\phi_{1}\left(u^{-1}(z), c\right)^{2}}\right) .
\end{aligned}
$$

We denote $F(z, c)=\phi_{1}\left(u^{-1}(z), c\right), G(z, c)=\frac{1}{\phi_{1}\left(u^{-1}(z), c\right)^{2}}$ and $h(z)=\varphi\left(u^{-1}(z)\right) \rho(z)$. Using the fact that

$$
(f \circ g)^{\prime}=\left(f^{\prime} \circ g\right) g^{\prime}, \quad(f \circ g)^{\prime \prime}=\left(f^{\prime \prime} \circ g\right)\left(g^{\prime}\right)^{2}+\frac{(f \circ g)^{\prime} g^{\prime \prime}}{g^{\prime}},
$$

we deduce from Lemma 11.1 and Lemma 11.2 that

$$
\begin{aligned}
& \rho(c) \int_{0}^{1} \frac{1}{\phi^{2}(z, c)} \int_{x_{0}}^{z}\left(e^{i t(u(y)-c) \alpha}-1\right) \partial_{y}^{2}(\varphi(y) \rho(u(y))) \phi_{1}(y, c) d y d z \\
& =\rho(c) \mathbb{T}\left(\left(u^{-1}\right)^{\prime}, h^{\prime \prime},\left(e^{i t(z-c) \alpha}-1\right) \frac{1}{\left(u^{-1}\right)^{\prime}} F, G\right) \\
& -\rho(c) \mathbb{T}\left(\left(u^{-1}\right)^{\prime}, h^{\prime},\left(e^{i t(z-c) \alpha}-1\right) \frac{\left(u^{-1}\right)^{\prime \prime}}{\left(\left(u^{-1}\right)^{\prime}\right)^{2}} F, G\right) \\
& =\rho(c) \partial_{c} \mathbb{T}\left(\left(u^{-1}\right)^{\prime}, h^{\prime},\left(e^{i t(z-c) \alpha}-1\right) \frac{1}{\left(u^{-1}\right)^{\prime}} F, G\right) \\
& -\rho(c) \mathbb{T}\left(\left(u^{-1}\right)^{\prime}, h^{\prime},\left(e^{i t(z-c) \alpha}-1\right)\left(\partial_{y}+\partial_{c}\right)\left(\frac{1}{\left(u^{-1}\right)^{\prime}} F\right), G\right) \\
& -\rho(c) \mathbb{T}\left(\left(u^{-1}\right)^{\prime}, h^{\prime},\left(e^{i t(z-c) \alpha}-1\right) \frac{1}{\left(u^{-1}\right)^{\prime}} F,\left(\partial_{z}+\partial_{c}\right) G\right) \\
& -\rho(c) \mathbb{T}\left(\left(u^{-1}\right)^{\prime \prime}, h^{\prime},\left(e^{i t(z-c) \alpha}-1\right) \frac{1}{\left(u^{-1}\right)^{\prime}} F, G\right) \\
& +\mathbb{B}_{0}\left(\left(u^{-1}\right)^{\prime}, h^{\prime},\left(e^{i t(z-c) \alpha}-1\right) \frac{1}{\left(u^{-1}\right)^{\prime}} F, G\right) \\
& +\mathbb{B}_{1}\left(\left(u^{-1}\right)^{\prime}, h^{\prime},\left(e^{i t(z-c) \alpha}-1\right) \frac{1}{\left(u^{-1}\right)^{\prime}} F, G\right) \\
& -\rho(c) \partial_{c} \mathbb{T}\left(\left(u^{-1}\right)^{\prime}, h,\left(e^{i t(z-c) \alpha}-1\right) \frac{\left(u^{-1}\right)^{\prime \prime}}{\left(\left(u^{-1}\right)^{\prime}\right)^{2}} F, G\right) \\
& +\rho(c) \mathbb{T}\left(\left(u^{-1}\right)^{\prime}, h,\left(e^{i t(z-c) \alpha}-1\right)\left(\partial_{z}+\partial_{c}\right)\left(\frac{\left(u^{-1}\right)^{\prime \prime}}{\left(\left(u^{-1}\right)^{\prime}\right)^{2}} F\right), G\right) \\
& +\rho(c) \mathbb{T}\left(\left(u^{-1}\right)^{\prime}, h,\left(e^{i t(z-c) \alpha}-1\right) \frac{\left(u^{-1}\right)^{\prime \prime}}{\left(\left(u^{-1}\right)^{\prime}\right)^{2}} F,\left(\partial_{z}+\partial_{c}\right) G\right) \\
& +\rho(c) \mathbb{T}\left(\left(u^{-1}\right)^{\prime \prime}, h,\left(e^{i t(z-c) \alpha}-1\right) \frac{\left(u^{-1}\right)^{\prime \prime}}{\left(\left(u^{-1}\right)^{\prime}\right)^{2}} F, G\right) \\
& -\mathbb{B}_{0}\left(\left(u^{-1}\right)^{\prime}, h,\left(e^{i t(z-c) \alpha}-1\right) \frac{\left(u^{-1}\right)^{\prime \prime}}{\left(\left(u^{-1}\right)^{\prime}\right)^{2}} F, G\right) \\
& -\mathbb{B}_{1}\left(\left(u^{-1}\right)^{\prime}, h,\left(e^{i t(z-c) \alpha}-1\right) \frac{\left(u^{-1}\right)^{\prime \prime}}{\left(\left(u^{-1}\right)^{\prime}\right)^{2}} F, G\right) .
\end{aligned}
$$


This gives

$$
\begin{aligned}
& \rho(c) \int_{0}^{1} \frac{1}{\phi^{2}(z, c)} \int_{x_{0}}^{z}\left(e^{i t(u(y)-c) \alpha}-1\right) \partial_{y}^{2}(\varphi(y) \rho(u(y))) \phi_{1}(y, c) d y d z \\
& =\rho(c) \partial_{c} \mathbb{T}\left(\left(u^{-1}\right)^{\prime}, h^{\prime},\left(e^{i t(z-c) \alpha}-1\right) \frac{1}{\left(u^{-1}\right)^{\prime}} F, G\right) \\
& \quad+\mathbb{B}_{0}\left(\left(u^{-1}\right)^{\prime}, h^{\prime},\left(e^{i t(z-c) \alpha}-1\right) \frac{1}{\left(u^{-1}\right)^{\prime}} F, G\right)+\mathbb{B}_{1}\left(\left(u^{-1}\right)^{\prime}, h^{\prime},\left(e^{i t(z-c) \alpha}-1\right) \frac{1}{\left(u^{-1}\right)^{\prime}} F, G\right) \\
& \quad+\Xi_{1}(h)+\rho(c) \partial_{c} \Xi_{2}(h),
\end{aligned}
$$

where

$$
\begin{aligned}
\Xi_{1}(h)= & -e^{-i t \alpha c} \mathcal{B}\left(h e^{i t z} \frac{\left(u^{-1}\right)^{\prime \prime}}{\left(\left(u^{-1}\right)^{\prime}\right)^{2}}\right)+\mathcal{B}\left(h \frac{\left(u^{-1}\right)^{\prime \prime}}{\left(\left(u^{-1}\right)^{\prime}\right)^{2}}\right) \\
& +\rho(c) e^{-i t \alpha c} \mathcal{L}_{2}\left(h e^{i t z} \frac{\left(u^{-1}\right)^{\prime \prime}}{\left(\left(u^{-1}\right)^{\prime}\right)^{2}}\right)-\rho(c) \mathcal{L}_{2}\left(h \frac{\left(u^{-1}\right)^{\prime \prime}}{\left(\left(u^{-1}\right)^{\prime}\right)^{2}}\right) \\
& +\rho(c) e^{-i t \alpha c} \mathcal{L}_{1}\left(h e^{i t z}\left(\frac{\left(u^{-1}\right)^{\prime \prime}}{\left(\left(u^{-1}\right)^{\prime}\right)^{2}}\right)-\rho(c) \mathcal{L}_{1}\left(h\left(\frac{\left(u^{-1}\right)^{\prime \prime}}{\left(\left(u^{-1}\right)^{\prime}\right)^{2}}\right)\right.\right. \\
& +\rho(c) e^{-i t \alpha c} \mathcal{L}_{3}\left(h e^{i t z} \frac{1}{\left(u^{-1}\right)^{\prime}}\right)-\rho(c) \mathcal{L}_{3}\left(h \frac{1}{\left(u^{-1}\right)^{\prime}}\right) \\
& -e^{-i t \alpha c} \mathcal{B}_{1}\left(h(z) e^{i t z} \frac{1}{\left(u^{-1}\right)^{\prime}}\right)+\mathcal{B}_{1}\left(h(z) \frac{1}{\left(u^{-1}\right)^{\prime}}\right) \\
& +\rho(c) e^{-i t \alpha c} \mathcal{L}_{1}\left(h(z) e^{i t z}\left(\frac{1}{\left(u^{-1}\right)^{\prime}}\right)^{\prime \prime}\right)-\rho(c) \mathcal{L}_{1}\left(h\left(\frac{1}{\left(u^{-1}\right)^{\prime}}\right)^{\prime \prime}\right)
\end{aligned}
$$

and

$$
\begin{aligned}
\Xi_{2}(h)=e^{-i t \alpha c} \mathcal{L}_{1}\left(h(z) e^{i t z} \frac{\left(u^{-1}\right)^{\prime \prime}}{\left(\left(u^{-1}\right)^{\prime}\right)^{2}}\right)-\mathcal{L}_{1}\left(h \frac{\left(u^{-1}\right)^{\prime \prime}}{\left(\left(u^{-1}\right)^{\prime}\right)^{2}}\right) \\
\quad-e^{-i t \alpha c} \mathcal{L}_{2}\left(h e^{i t z} \frac{1}{\left(u^{-1}\right)^{\prime}}\right)-\mathcal{L}_{2}\left(h \frac{1}{\left(u^{-1}\right)^{\prime}}\right) \\
\quad-e^{-i t \alpha c} \mathcal{L}_{2}\left(h e^{i t z}\left(\frac{1}{\left(u^{-1}\right)^{\prime}}\right)^{\prime}\right)-\mathcal{L}_{2}\left(h\left(\frac{1}{\left(u^{-1}\right)^{\prime}}\right)^{\prime}\right) .
\end{aligned}
$$

By Lemma 11.10 and Lemma 11.9, we have

$$
\left\|\Xi_{1}(h)\right\|_{L^{2}}+\left\|\Xi_{2}(h)\right\|_{L^{2}} \leq C\|h\|_{L^{2}} \leq C\|\varphi\|_{L^{2}} .
$$

Using Lemma 11.3, we write

$$
\begin{aligned}
& \mathbb{B}_{0}\left(\left(u^{-1}\right)^{\prime}, h^{\prime},\left(e^{i t(z-c) \alpha}-1\right) \frac{1}{\left(u^{-1}\right)^{\prime}} F, G\right) \\
& \quad+\mathbb{B}_{1}\left(\left(u^{-1}\right)^{\prime}, h^{\prime},\left(e^{i t(z-c) \alpha}-1\right) \frac{1}{\left(u^{-1}\right)^{\prime}} F, G\right) \\
& =\partial_{c} \mathbb{B}_{0}\left(\left(u^{-1}\right)^{\prime}, h\left(e^{i t(z-c) \alpha}-1\right) \frac{1}{\left(u^{-1}\right)^{\prime}}, F, G\right) \\
& \quad+\partial_{c} \mathbb{B}_{1}\left(\left(u^{-1}\right)^{\prime}, h\left(e^{i t(z-c) \alpha}-1\right) \frac{1}{\left(u^{-1}\right)^{\prime}}, F, G\right) \\
& -\mathbb{B}_{0}\left(\left(u^{-1}\right)^{\prime}, h,\left(e^{i t(z-c) \alpha}-1\right) \frac{1}{\left(u^{-1}\right)^{\prime}} F, \partial_{c} G\right)
\end{aligned}
$$


LINEAR INVISCID DAMPING FOR A CLASS OF MONOTONE SHEAR FLOW IN SOBOLEV SPACES 37

$$
\begin{aligned}
& -\mathbb{B}_{1}\left(\left(u^{-1}\right)^{\prime}, h,\left(e^{i t(z-c) \alpha}-1\right) \frac{1}{\left(u^{-1}\right)^{\prime}} F, \partial_{c} G\right) \\
& -\mathbb{B}_{0}\left(\left(u^{-1}\right)^{\prime}, h\left(e^{i t(z-c) \alpha}-1\right) \frac{1}{\left(u^{-1}\right)^{\prime}},\left(\partial_{z}+\partial_{c}\right) F, \partial_{c} G\right) \\
& -\mathbb{B}_{1}\left(\left(u^{-1}\right)^{\prime}, h\left(e^{i t(z-c) \alpha}-1\right) \frac{1}{\left(u^{-1}\right)^{\prime}},\left(\partial_{z}+\partial_{c}\right) F, \partial_{c} G\right) \\
& -\mathbb{B}_{0}\left(\left(u^{-1}\right)^{\prime}, h\left(e^{i t(z-c) \alpha}-1\right)\left(\frac{1}{\left(u^{-1}\right)^{\prime}}\right)^{\prime}, F, \partial_{c} G\right) \\
& -\mathbb{B}_{1}\left(\left(u^{-1}\right)^{\prime}, h\left(e^{i t(z-c) \alpha}-1\right)\left(\frac{1}{\left(u^{-1}\right)^{\prime}}\right)^{\prime}, F, \partial_{c} G\right) \\
& +\frac{\left(u^{-1}\right)^{\prime}(u(0)) G(u(0), c)}{c-u(0)} \int_{u(0)}^{c} h(z)\left(e^{i t(z-c) \alpha}-1\right) \frac{1}{\left(u^{-1}\right)^{\prime}} F(z, c) d z \\
& +\frac{\left(u^{-1}\right)^{\prime}(u(1)) G(u(1), c)}{u(1)-c} \int_{c}^{u(1)} h(z)\left(e^{i t(z-c) \alpha}-1\right) \frac{1}{\left(u^{-1}\right)^{\prime}} F(z, c) d z \\
& \triangleq \partial_{c} \Xi_{4}(h)+\Xi_{3}(h) .
\end{aligned}
$$

It follows from Lemma 11.8 that

$$
\left\|\Xi_{3}(h)\right\|_{L^{2}} \leq C\|\varphi\|_{L^{2}}, \quad\left\|\Xi_{4}(h)\right\|_{L^{2}} \leq \frac{C}{\alpha}\|\varphi\|_{L^{2}}
$$

Using Lemma 11.1, we write

$$
\begin{aligned}
\rho(c) & \partial_{c} \mathbb{T}\left(\left(u^{-1}\right)^{\prime}, h^{\prime},\left(e^{i t(z-c) \alpha}-1\right) \frac{1}{\left(u^{-1}\right)^{\prime}} F, G\right) \\
= & \rho(c) \partial_{c}^{2} \mathbb{T}\left(\left(u^{-1}\right)^{\prime}, h\left(e^{i t(z-c) \alpha}-1\right) \frac{1}{\left(u^{-1}\right)^{\prime}}, F, G\right) \\
& -\rho(c) \partial_{c} \mathbb{T}\left(\left(u^{-1}\right)^{\prime}, h,\left(e^{i t(z-c) \alpha}-1\right)\left(\partial_{c}+\partial_{y}\right)\left(\frac{1}{\left(u^{-1}\right)^{\prime}} F\right), G\right) \\
& -\rho(c) \partial_{c} \mathbb{T}\left(\left(u^{-1}\right)^{\prime}, h,\left(e^{i t(z-c) \alpha}-1\right) \frac{1}{\left(u^{-1}\right)^{\prime}} F,\left(\partial_{c}+\partial_{z}\right) G\right) \\
& -\rho(c) \partial_{c} \mathbb{T}\left(\left(u^{-1}\right)^{\prime \prime}, h,\left(e^{i t(z-c) \alpha}-1\right) \frac{1}{\left(u^{-1}\right)^{\prime}} F, G\right) \\
& +\rho(c) \partial_{c} \frac{1}{\rho} \mathbb{B}_{0}\left(\left(u^{-1}\right)^{\prime}, h,\left(e^{i t(z-c) \alpha}-1\right) \frac{1}{\left(u^{-1}\right)^{\prime}} F, G\right) \\
& +\rho(c) \partial_{c} \frac{1}{\rho} \mathbb{B}_{1}\left(\left(u^{-1}\right)^{\prime}, h,\left(e^{i t(z-c) \alpha}-1\right) \frac{1}{\left(u^{-1}\right)^{\prime}} F, G\right) \\
= & \rho(c) \partial_{c}^{2}\left[\mathcal{L}_{1}, \rho\right]\left(\varphi\left(e^{i t(z-c) \alpha}-1\right) \frac{1}{\left(u^{-1}\right)^{\prime}}\right)+\rho(c) \partial_{c}^{2}\left(\rho \mathcal{L}_{1}\left(\varphi\left(e^{i t(z-c) \alpha}-1\right) \frac{1}{\left(u^{-1}\right)^{\prime}}\right)\right) \\
& -\rho(c) \partial_{c} \mathcal{L}_{2}\left(h\left(e^{i t(z-c) \alpha}-1\right) \frac{1}{\left(u^{-1}\right)^{\prime}}\right)-\rho(c) \partial_{c} \mathcal{L}_{1}\left(h\left(e^{i t(z-c) \alpha}-1\right)\left(\frac{1}{\left(u^{-1}\right)^{\prime}}\right)^{\prime}\right) \\
& +\rho(c) \partial_{c} \frac{1}{\rho} \mathcal{B}\left(h\left(e^{i t(z-c) \alpha}-1\right) \frac{1}{\left(u^{-1}\right)^{\prime}}\right) \\
\triangleq & \rho(c) \partial_{c} \Xi_{5}(\varphi)+\rho \partial_{c}^{2}\left(\rho \Xi_{6}(\varphi)\right)+\rho \partial_{c} \Xi_{7}(h) .
\end{aligned}
$$

This together with Lemma 11.11, Lemma 11.10 and Lemma 11.9 shows that

$$
\left\|\Xi_{5}(\varphi)\right\|_{L^{2}}+\left\|\Xi_{6}(\varphi)\right\|_{L^{2}}+\left\|\Xi_{7}(h)\right\|_{L^{2}} \leq C\|\varphi\|_{L^{2}} .
$$


Thus, we obtain

$$
\begin{aligned}
& \int_{0}^{1} \rho(u(y)) \partial_{y}^{2} \mathbf{T}(\rho \mu(\cdot))(t, y) \varphi(y) d y \\
& =-\int_{u(0)}^{u(1)} \mu(c)\left(\Xi_{1}(h)+\rho(c) \partial_{c} \Xi_{2}(h)+\Xi_{3}(h)+\partial_{c} \Xi_{4}(h)\right. \\
& \left.\quad+\rho(c) \partial_{c} \Xi_{5}(\varphi)+\rho \partial_{c}^{2}\left(\rho \Xi_{6}(\varphi)\right)+\rho \partial_{c} \Xi_{7}(h)\right) d c \\
& =-\int_{u(0)}^{u(1)} \mu(c) \Xi_{1}(h)-\partial_{c}(\rho \mu) \Xi_{2}(h)+\mu(c) \Xi_{3}(h)-\Xi_{4}(h) \partial_{c} \mu \\
& \quad-\partial_{c}(\rho \mu) \Xi_{5}(\varphi)+\rho(c) \partial_{c}^{2}(\rho \mu) \Xi_{6}(\varphi)-\partial_{c}(\rho \mu) \Xi_{7}(h) d c,
\end{aligned}
$$

which along with (9.1)-(9.3) and Proposition 8.1 yields

$$
\left\|\rho(u(y)) \partial_{y}^{2} \mathbf{T}(\rho \mu)(t, y)\right\|_{L^{2}} \leq C\left\|\widehat{\omega}_{0}(\alpha, \cdot)\right\|_{H^{2}} .
$$

This implies

$$
\left\|\rho(u(\cdot)) \partial_{y}^{2} W(t)\right\|_{H_{x}^{-1} H_{y}^{2}} \leq C\left\|\omega_{0}\right\|_{H_{x}^{-1} H_{y}^{2}} .
$$

The proof of the proposition is completed.

\section{Proof of Theorem 1.1}

This section is devoted to the proof of Theorem 1.1.

10.1. Decay estimates. With the uniform Sobolev estimates of the vorticity, the decay estimates of the velocity are similar to [16]. For the completeness, we present a proof. By the elliptical estimate, we get

$$
\|V\|_{L^{2}} \leq C\|\omega\|_{H^{-1}}
$$

We get by duality that

$$
\begin{aligned}
& \|V(t)\|_{L^{2}} \leq \sup _{\varphi \in C_{0}^{\infty},\|\varphi\|_{H^{1}} \leq 1}\left|\int_{0}^{1} \int_{-\pi}^{\pi} \varphi \omega(t) d x d y\right| \\
& \leq C \sup _{\varphi \in C_{0}^{\infty},\|\varphi\|_{H^{1}} \leq 1}\left|\sum_{|\alpha| \neq 0} \int_{0}^{1} \overline{\widehat{\varphi}(\alpha, y)} \widehat{W}(t, \alpha, y) e^{-i \alpha u(y) t} d y\right| .
\end{aligned}
$$

Integration by parts gives

$$
\begin{aligned}
\int_{0}^{1} \overline{\widehat{\varphi}(\alpha, y)} \widehat{W}(t, \alpha, y) e^{-i \alpha u(y) t} d y= & \left.\frac{e^{-i \alpha u(y) t}}{-i \alpha t u^{\prime}(y)} \overline{\widehat{\varphi}(\alpha, y)} \widehat{W}(t, \alpha, y)\right|_{y=0} ^{1} \\
& -\int_{0}^{1} \frac{e^{-i \alpha u(y) t}}{-i \alpha t} \partial_{y}\left(\frac{\widehat{\varphi}(\alpha, y) \hat{W}(t, \alpha, y)}{u^{\prime}(y)}\right) d y \\
= & -\int_{0}^{1} \frac{e^{-i \alpha u(y) t}}{-i \alpha t} \partial_{y}\left(\frac{\widehat{\widehat{\varphi}(\alpha, y)} \widehat{W}(t, \alpha, y)}{u^{\prime}(y)}\right) d y
\end{aligned}
$$

from which and Proposition 9.1, we infer that

$$
\begin{aligned}
\|V(t)\|_{L^{2}} & \leq C t^{-1} \sup _{\varphi \in C_{0}^{\infty},\|\varphi\|_{H^{1}} \leq 1}\|W(t)\|_{H_{x}^{-1} H_{y}^{1}}\|\varphi\|_{L_{x}^{2} H_{y}^{1}} \\
& \leq C t^{-1}\left\|\omega_{0}\right\|_{H_{x}^{-1} H_{y}^{1}} .
\end{aligned}
$$


Also we get from (10.1) that

$$
\begin{aligned}
\|V(t)\|_{L^{2}} & \leq C \sup _{\varphi \in C_{0}^{\infty},\|\varphi\|_{H^{1}} \leq 1}\|W(t)\|_{H_{x}^{-1} L_{y}^{2}}\|\varphi\|_{H_{x}^{1} L_{y}^{2}} \\
& \leq C\left\|\omega_{0}\right\|_{H_{x}^{-1} L_{y}^{2}} .
\end{aligned}
$$

Recall that $-\triangle V^{2}=\partial_{x} \omega$ and $V^{2}(x, 0)=V^{2}(1, y)=0$. We define

$$
-\Delta \vartheta=V^{2}, \quad \vartheta(t, x, 0)=\vartheta(t, x, 1)=0 .
$$

We get by the elliptic estimate that

$$
\|\vartheta\|_{H^{2}} \leq C\left\|V^{2}\right\|_{L^{2}}
$$

Integration by parts gives

$$
\begin{aligned}
&\left\|V^{2}\right\|_{L^{2}}^{2}= \iint \partial_{x} \omega \vartheta d x d y=\sum_{|\alpha| \neq 0} \int_{0}^{1} i \alpha e^{-i \alpha u(y) t} \widehat{W}(t, \alpha, y) \overline{\widehat{\vartheta}}(t, \alpha, y) d y \\
&= \sum_{|\alpha| \neq 0} \int_{0}^{1} \frac{e^{-i \alpha u(y) t}}{t} \partial_{y}\left(\frac{\widehat{W}(t, \alpha, y) \overline{\widehat{\vartheta}}(t, \alpha, y)}{u^{\prime}(y)}\right) d y \\
&=\left.\sum_{|\alpha| \neq 0} \frac{e^{-i \alpha u(y) t}}{i \alpha t^{2} u^{\prime}} \partial_{y}\left(\frac{\widehat{W}(t, \alpha, y) \overline{\widehat{\vartheta}}(t, \alpha, y)}{u^{\prime}(y)}\right)\right|_{y=0} ^{1} \\
&-\sum_{|\alpha| \neq 0} \int_{0}^{1} \frac{e^{-i \alpha u(y) t}}{i \alpha t^{2}} \partial_{y}\left(\frac { 1 } { u ^ { \prime } } \partial _ { y } \left(\frac{\widehat{W}(t, \alpha, y)}{u^{\prime}(y)} \overline{\widehat{\vartheta}}(t, \alpha, y)\right.\right. \\
&
\end{aligned}
$$

By Sobolev embedding and Proposition 9.1, the first term on the right hand side is bounded by

$$
C t^{-2} \sum_{|\alpha| \neq 0} \frac{1}{\alpha}\|\widehat{W}(t, \alpha, \cdot)\|_{H_{y}^{1}}\|\vartheta(t, \alpha, \cdot)\|_{H_{y}^{2}} \leq C t^{-2}\left\|\omega_{0}\right\|_{H_{x}^{-1} H_{y}^{2}}\left\|V^{2}\right\|_{L^{2}} .
$$

Using Proposition 9.1 again and Hardy inequality, we get

$$
\begin{aligned}
\left\|\partial_{y}\left(\frac{1}{u^{\prime}} \partial_{y}\left(\frac{\widehat{W}(t, \alpha, y) \overline{\widehat{\vartheta}}(t, \alpha, y)}{u^{\prime}(y)}\right)\right)\right\|_{L_{y}^{1}} \leq & C\left\|\rho(u(y)) \partial_{y}^{2} \widehat{W}(t, \alpha, y)\right\|_{L_{y}^{2}}\left\|\frac{\vartheta(t, \alpha, y)}{\rho(u(y))}\right\|_{L_{y}^{2}} \\
& +C\left\|\partial_{y} \widehat{W}(t, \alpha, y)\right\|_{L_{y}^{2}}\|\vartheta(t, \alpha, y)\|_{H_{y}^{1}} \\
& +C\|\widehat{W}(t, \alpha, y)\|_{L_{y}^{2}}\|\vartheta(t, \alpha, y)\|_{H_{y}^{2}} \\
\leq & C\left\|\omega_{0}(\alpha, \cdot)\right\|_{H_{y}^{2}}\|\vartheta(t, \alpha, y)\|_{H_{y}^{2}} .
\end{aligned}
$$

This implies that the second term is bounded by

$$
C t^{-2}\left\|\omega_{0}\right\|_{H_{x}^{-1} H_{y}^{2}}\left\|V^{2}\right\|_{L^{2}}
$$

Thus, we show that

$$
\left\|V^{2}\right\|_{L^{2}} \leq C t^{-2}\left\|\omega_{0}\right\|_{H_{x}^{-1} H_{y}^{2}}
$$


10.2. Scattering. We only prove $H^{1}$ scattering. The proof of $L^{2}$ scattering is similar. Suppose that $\omega_{0} \in H_{x}^{-1} H_{y}^{1}$. Let $\left\{\omega_{0}^{(n)}\right\}_{n \geq 1} \subseteq H_{x}^{-1} H_{y}^{2}$ be a sequence such that $\omega_{0}^{(n)} \longrightarrow \omega_{0}$ in $H_{x}^{-1} H_{y}^{1}$. Let $\omega^{(n)}(t, x, y)$ be the solution to linearized Euler equation with initial data $\omega_{0}^{(n)}(x, y)$. Then $\omega^{(n)} \longrightarrow \omega$ in $L_{t}^{\infty}\left(H_{x}^{-1} H_{y}^{1}\right)$. Thanks to $W_{t}^{(n)}=u^{\prime \prime}(y) \partial_{x} \psi^{(n)}$, we get

$$
W^{(n)}(t, x, y)=\omega^{(n)}(0, x, y)-\int_{0}^{t} u^{\prime \prime}(y) \partial_{x} \psi^{(n)}(s, x+s u(y), y) d s .
$$

By the decay estimate, we have

$$
\left\|\partial_{x} \psi^{(n)}(s, x, y)\right\|_{L_{x, y}^{2}} \leq \frac{C}{\langle t\rangle^{2}}\left\|\omega_{0}^{(n)}\right\|_{H_{x}^{-1} H_{y}^{2}}
$$

Letting $t \rightarrow \infty$, we get

$$
W^{(n)}(t, x, y) \longrightarrow \omega^{(n)}(0, x, y)-\int_{0}^{\infty} u^{\prime \prime}(y) \partial_{x} \psi^{(n)}(s, x+s u(y), y) d s=\omega_{\infty}^{(n)} \quad \text { in } H_{x}^{-1} L_{y}^{2} .
$$

By Proposition 9.1, $\left\{W^{(n)}\right\}_{n \geq 1}$ is a Cauchy sequence in $L_{t}^{\infty}\left(H_{x}^{-1} H_{y}^{1}\right)$. Thus, $\omega_{\infty}^{(n)}$ is a Cauchy sequence in $H_{x}^{-1} H_{y}^{1}$, then $\omega^{\infty}=\lim _{n \rightarrow \infty} \omega_{\infty}^{(n)} \in H_{x}^{-1} H_{y}^{1}$.

\section{Appendix}

11.1. Multilinear singular integral operators. The multilinear operator $\mathbb{T}$ is defined by

$$
\mathbb{T}(f, g, F, G)(c) \stackrel{\text { def }}{=} \text { p.v. } \int_{u(0)}^{u(1)} \frac{\int_{c}^{c^{\prime}} g(z) F(z, c) d z}{\left(c-c^{\prime}\right)^{2}} G\left(c^{\prime}, c\right) f\left(c^{\prime}\right) d c^{\prime},
$$

where $f, g$ are functions defined on $D_{0}$ and $F, G$ are functions defined on $D_{0} \times D_{0}$.

The multilinear operator $\mathbb{B}_{0}$ and $\mathbb{B}_{1}$ are defined by

$$
\begin{aligned}
& \mathbb{B}_{0}(f, g, F, G)(c) \stackrel{\text { def }}{=} \frac{(u(1)-c) f(u(0))}{c-u(0)} \int_{u(0)}^{c} g(z) F(z, c) G(u(0), c) d z, \\
& \mathbb{B}_{1}(f, g, F, G)(c) \stackrel{\text { def }}{=} \frac{(c-u(0)) f(u(1))}{u(1)-c} \int_{c}^{u(1)} g(z) F(z, c) G(u(1), c) d z .
\end{aligned}
$$

Lemma 11.1. It holds that

$$
\begin{aligned}
\partial_{c}(\rho(c) \mathbb{T}(f, g, F, G)(c))= & \rho(c) \mathbb{T}\left(f, g^{\prime}, F, G\right)(c)+\rho(c) \mathbb{T}\left(f, g,\left(\partial_{z}+\partial_{c}\right) F, G\right)(c) \\
& +\rho(c) \mathbb{T}\left(f, g, F,\left(\partial_{z}+\partial_{c}\right) G\right)(c) \\
& +\rho(c) \mathbb{T}\left(f^{\prime}, g, F, G\right)(c)+\rho^{\prime}(c) \mathbb{T}(f, g, F, G)(c) \\
& -\mathbb{B}_{0}(f, g, F, G)(c)-\mathbb{B}_{1}(f, g, F, G)(c) .
\end{aligned}
$$

Proof. Thanks to

$$
\rho(c)-\rho\left(c^{\prime}\right)=\left(c^{\prime}-c\right)\left(c^{\prime}+c-u(0)-u(1)\right),
$$

we get

$$
\begin{aligned}
& \rho(c) \mathbb{T}(f, g, F, G)(c) \\
& =\text { p.v. } \int_{u(0)}^{u(1)} \frac{\left(c^{\prime}+c-u(0)-u(1)\right) \int_{c}^{c^{\prime}} g(z) F(z, c) d z}{c^{\prime}-c} G\left(c^{\prime}, c\right) f\left(c^{\prime}\right) d c^{\prime} \\
& \quad+\text { p.v. } \int_{u(0)}^{u(1)} \frac{\int_{c}^{c^{\prime}} g(z) F(z, c) d z}{\left(c^{\prime}-c\right)^{2}} G\left(c^{\prime}, c\right) f\left(c^{\prime}\right) \rho\left(c^{\prime}\right) d c^{\prime} .
\end{aligned}
$$


LINEAR INVISCID DAMPING FOR A CLASS OF MONOTONE SHEAR FLOW IN SOBOLEV SPACES 41

A direct calculation shows

$$
\begin{aligned}
& \partial_{c}\left(\frac{\int_{c}^{c^{\prime}} g(z) F(z, c) d z}{c^{\prime}-c}\right) \\
& =-\partial_{c^{\prime}}\left(\frac{\int_{c}^{c^{\prime}} g(z) F(z, c) d z}{c^{\prime}-c}\right)+\frac{\int_{c}^{c^{\prime}} g^{\prime}(z) F(z, c) d z}{c^{\prime}-c}+\frac{\int_{c}^{c^{\prime}} g(z)\left(\partial_{c}+\partial_{z}\right) F(z, c) d z}{c^{\prime}-c},
\end{aligned}
$$

and

$$
\begin{aligned}
& \partial_{c}\left(\frac{\int_{c}^{c^{\prime}} g(z) F(z, c) d z}{(z-c)^{2}}\right) \\
& =-\partial_{c^{\prime}}\left(\frac{\int_{c}^{c^{\prime}} g(z) F(z, c) d z}{\left(c^{\prime}-c\right)^{2}}\right)+\frac{\int_{c}^{c^{\prime}} g^{\prime}(z) F(z, c) d z}{\left(c^{\prime}-c\right)^{2}}+\frac{\int_{c}^{c^{\prime}} g(z)\left(\partial_{c}+\partial_{z}\right) F(z, c) d y}{\left(c^{\prime}-c\right)^{2}} .
\end{aligned}
$$

Thus, we obtain

$$
\begin{aligned}
& \partial_{c}(\rho(c) \mathbb{T}(f, g, F, G)(c)) \\
= & \text { p.v. } \int_{u(0)}^{u(1)} \frac{\int_{c}^{c^{\prime}} g(z) F(z, c) d z}{\left(c^{\prime}-c\right)}\left(\partial_{c}+\partial_{c^{\prime}}\right)\left(G\left(c^{\prime}, c\right) f\left(c^{\prime}\right)\left(c^{\prime}+c-u(0)-u(1)\right)\right) d c^{\prime} \\
& + \text { p.v. } \int_{u(0)}^{u(1)} \frac{\int_{c}^{c^{\prime}} g(z) F(z, c) d z}{\left(c^{\prime}-c\right)^{2}}\left(\partial_{c}+\partial_{c^{\prime}}\right)\left(G\left(c^{\prime}, c\right) f\left(c^{\prime}\right) \rho\left(c^{\prime}\right)\right) d c^{\prime} \\
& + \text { p.v. } \int_{u(0)}^{u(1)} \frac{\int_{c}^{c^{\prime}} g(z)\left(\partial_{c}+\partial_{z}\right) F(z, c) d z}{\left(c^{\prime}-c\right)} G\left(c^{\prime}, c\right) f\left(c^{\prime}\right)\left(c^{\prime}+c-u(0)-u(1)\right) d c^{\prime} \\
& + \text { p.v. } \int_{u(0)}^{u(1)} \frac{\int_{c}^{c^{\prime}} g(z)\left(\partial_{c}+\partial_{z}\right) F(z, c) d z}{\left(c^{\prime}-c\right)^{2}} G\left(c^{\prime}, c\right) f\left(c^{\prime}\right) \rho\left(c^{\prime}\right) d c^{\prime} \\
& + \text { p.v. } \int_{u(0)}^{u(1)} \frac{\int_{c}^{c^{\prime}} g^{\prime}(z) F(z, c) d z}{\left(c^{\prime}-c\right)} G\left(c^{\prime}, c\right) f\left(c^{\prime}\right)\left(c^{\prime}+c-u(0)-u(1)\right) d c^{\prime} \\
& + \text { p.v. } \int_{u(0)}^{u(1)} \frac{\int_{c}^{c^{\prime}} g^{\prime}(z) F(z, c) d z}{\left(c^{\prime}-c\right)^{2}} G\left(c^{\prime}, c\right) f\left(c^{\prime}\right) \rho\left(c^{\prime}\right) d c^{\prime} \\
& -\frac{(c-u(0)) f(u(1))}{u(1)-c} \int_{c}^{u(1)} g(z) F(z, c) G(u(1), c) d z \\
& +\frac{(c-u(1)) f(u(0))}{c-u(0)} \int_{u(0)}^{c} g(z) F(z, c) G(u(0), c) d z .
\end{aligned}
$$

Then the lemma follows by using

$$
\begin{aligned}
& \left(c^{\prime}-c\right)\left(\partial_{c}+\partial_{c^{\prime}}\right)\left(\left(c^{\prime}+c-u(0)-u(1)\right) f\left(c^{\prime}\right)\right)+\partial_{c^{\prime}}\left(f\left(c^{\prime}\right) \rho\left(c^{\prime}\right)\right) \\
& =\rho^{\prime}(c) f\left(c^{\prime}\right)+\rho(c) f^{\prime}\left(c^{\prime}\right)
\end{aligned}
$$

and $\rho(c)-\rho\left(c^{\prime}\right)=\left(c^{\prime}-c\right)\left(c^{\prime}+c-u(0)-u(1)\right)$.

By Lemma 11.1 and $\left(\partial_{z}+\partial_{c}\right) e^{i t \alpha(z-c)}=0$, we can deduce that

Lemma 11.2. It holds that

$$
\begin{aligned}
\rho(c) \mathbb{T}\left(f, g^{\prime}, e^{i t \alpha(z-c)} F, G\right)(c)= & \rho(c) \partial_{c}\left(e^{-i t \alpha c} \mathbb{T}\left(f, g e^{i t \alpha z}, F, G\right)\right)(c) \\
& -\rho(c) e^{-i t \alpha c} \mathbb{T}\left(f, g e^{i t \alpha z},\left(\partial_{z}+\partial_{c}\right) F, G\right)(c)
\end{aligned}
$$




$$
\begin{aligned}
& -\rho(c) e^{-i t \alpha c} \mathbb{T}\left(f, g e^{i t \alpha z}, F,\left(\partial_{z}+\partial_{c}\right) G\right)(c) \\
& -\rho(c) e^{-i t \alpha c} \mathbb{T}\left(f^{\prime}, g e^{i t \alpha z}, F, G\right)(c) \\
& +e^{-i t \alpha c} \mathbb{B}_{0}\left(f, g e^{i t \alpha z}, F, G\right)(c)+e^{-i t \alpha c} \mathbb{B}_{1}\left(f, g e^{i t \alpha z}, F, G\right)(c) .
\end{aligned}
$$

Lemma 11.3. It holds that

$$
\begin{aligned}
\partial_{c} \mathbb{B}_{0}(f, g, F, G)(c)= & \mathbb{B}_{0}\left(f, g, F, \partial_{c} G\right)(c)-\frac{f(u(0)) G(u(0), c)}{c-u(0)} \int_{u(0)}^{c} g(z) F(z, c) d z \\
& +\mathbb{B}_{0}\left(f, g^{\prime}, F, G\right)(c)+\mathbb{B}_{0}\left(f, g,\left(\partial_{z}+\partial_{c}\right) F, G\right)(c), \\
\partial_{c} \mathbb{B}_{1}(f, g, F, G)(c)= & \mathbb{B}_{1}\left(f, g, F, \partial_{c} G\right)(c)-\frac{f(u(1)) G(u(0), c)}{u(1)-c} \int_{c}^{u(1)} g(z) F(z, c) d z \\
& +\mathbb{B}_{1}\left(f, g^{\prime}, F, G\right)(c)+\mathbb{B}_{1}\left(f, g,\left(\partial_{z}+\partial_{c}\right) F, G\right)(c) .
\end{aligned}
$$

Proof. The lemma follows by a direct calculation.

Next let us introduce some linear operators. Let $\phi(y, c)$ be the solution of (4.1) given by Proposition 4.5 and $\phi_{1}(y, c)=\frac{\phi(y, c)}{u(y)-c}$. We define

$$
\begin{aligned}
\mathcal{L}_{1}(g)(c) \stackrel{\text { def }}{=} & \mathbb{T} \\
\mathcal{L}_{2}(g)(c) \stackrel{\text { def }}{=} & \mathbb{T}\left(\left(u^{-1}\right)^{\prime}, g, \phi_{1}, g,\left(\partial_{z}+\partial_{c}\right) \phi_{1}\left(u^{-1}, c\right), \frac{1}{\phi_{1}\left(u^{-1}, c\right)^{2}}\right)(c) \\
& +\mathbb{T}\left(\left(u^{-1}\right)^{\prime}, g, \phi_{1}\left(u^{-1}, c\right),\left(\partial_{z}+\partial_{c}\right)\left(\frac{1}{\phi_{1}\left(u^{-1}, c\right)^{2}}\right)\right)(c) \\
& +\mathbb{T}\left(\left(u^{-1}\right)^{\prime \prime}, g, \phi_{1}\left(u^{-1}, c\right), \frac{1}{\phi_{1}\left(u^{-1}, c\right)^{2}}\right)(c), \\
\mathcal{L}_{3}(g)(c) \stackrel{\text { def }}{=} & \mathbb{T}\left(\left(u^{-1}\right)^{\prime}, g,\left(\partial_{z}+\partial_{c}\right)^{2} \phi_{1}\left(u^{-1}, c\right), \frac{1}{\phi_{1}\left(u^{-1}, c\right)^{2}}\right)(c) \\
& +\mathbb{T}\left(\left(u^{-1}\right)^{\prime}, g, \phi_{1}\left(u^{-1}, c\right),\left(\partial_{z}+\partial_{c}\right)^{2}\left(\frac{1}{\phi_{1}\left(u^{-1}, c\right)^{2}}\right)\right)(c) \\
& +\mathbb{T}\left(\left(u^{-1}\right)^{\prime \prime \prime}, g, \phi_{1}\left(u^{-1}, c\right), \frac{1}{\phi_{1}\left(u^{-1}, c\right)^{2}}\right)(c) \\
& +2 \mathbb{T}\left(\left(u^{-1}\right)^{\prime \prime}, g,\left(\partial_{z}+\partial_{c}\right) \phi_{1}\left(u^{-1}, c\right), \frac{1}{\phi_{1}\left(u^{-1}, c\right)^{2}}\right)(c) \\
& +2 \mathbb{T}\left(\left(u^{-1}\right)^{\prime}, g,\left(\partial_{z}+\partial_{c}\right) \phi_{1}\left(u^{-1}, c\right),\left(\partial_{z}+\partial_{c}\right)\left(\frac{1}{\phi_{1}\left(u^{-1}, c\right)^{2}}\right)\right)(c) \\
& +2 \mathbb{T}\left(\left(u^{-1}\right)^{\prime \prime}, g, \phi_{1}\left(u^{-1}, c\right),\left(\partial_{z}+\partial_{c}\right)\left(\frac{1}{\phi_{1}\left(u^{-1}, c\right)^{2}}\right)\right)(c) .
\end{aligned}
$$

We also introduce the linear operators

$$
\begin{aligned}
\mathcal{B}(g)(c) \stackrel{\text { def }}{=} & \mathbb{B}_{0}\left(\left(u^{-1}\right)^{\prime}, g, \phi_{1}\left(u^{-1}, c\right), \frac{1}{\phi_{1}\left(u^{-1}, c\right)^{2}}\right)(c) \\
& +\mathbb{B}_{1}\left(\left(u^{-1}\right)^{\prime}, g, \phi_{1}\left(u^{-1}, c\right), \frac{1}{\phi_{1}\left(u^{-1}, c\right)^{2}}\right)(c), \\
\mathcal{B}_{1}(g)(c) \stackrel{\text { def }}{=} & \mathbb{B}_{0}\left(\left(u^{-1}\right)^{\prime}, g,\left(\partial_{z}+\partial_{c}\right) \phi_{1}\left(u^{-1}, c\right), \frac{1}{\phi_{1}\left(u^{-1}, c\right)^{2}}\right)(c)
\end{aligned}
$$


LINEAR INVISCID DAMPING FOR A CLASS OF MONOTONE SHEAR FLOW IN SOBOLEV SPACES 43

$$
\begin{aligned}
& +\mathbb{B}_{1}\left(\left(u^{-1}\right)^{\prime}, g,\left(\partial_{z}+\partial_{c}\right) \phi_{1}\left(u^{-1}, c\right), \frac{1}{\phi_{1}\left(u^{-1}, c\right)^{2}}\right)(c) \\
& +\mathbb{B}_{0}\left(\left(u^{-1}\right)^{\prime}, g, \phi_{1}\left(u^{-1}, c\right),\left(\partial_{z}+\partial_{c}\right)\left(\frac{1}{\phi_{1}\left(u^{-1}, c\right)^{2}}\right)\right)(c) \\
& +\mathbb{B}_{1}\left(\left(u^{-1}\right)^{\prime}, g, \phi_{1}\left(u^{-1}, c\right),\left(\partial_{z}+\partial_{c}\right)\left(\frac{1}{\phi_{1}\left(u^{-1}, c\right)^{2}}\right)\right)(c) \\
& +\mathbb{B}_{0}\left(\left(u^{-1}\right)^{\prime \prime}, g, \phi_{1}\left(u^{-1}, c\right), \frac{1}{\phi_{1}\left(u^{-1}, c\right)^{2}}\right)(c) \\
& +\mathbb{B}_{1}\left(\left(u^{-1}\right)^{\prime \prime}, g, \phi_{1}\left(u^{-1}, c\right), \frac{1}{\phi_{1}\left(u^{-1}, c\right)^{2}}\right)(c) .
\end{aligned}
$$

Lemma 11.4. It holds that

$$
\begin{aligned}
& \partial_{c}\left(\rho \mathcal{L}_{1}(g)\right)=\rho(c) \mathcal{L}_{1}\left(g^{\prime}\right)+\rho^{\prime}(c) \mathcal{L}_{1}(g)+\rho(c) \mathcal{L}_{2}(g)+\mathcal{B}(g), \\
& \rho \partial_{c} \mathcal{L}_{2}(g)=\rho \mathcal{L}_{2}\left(g^{\prime}\right)+\rho \mathcal{L}_{3}(g)-\mathcal{B}_{1}(g)
\end{aligned}
$$

and

$$
\begin{aligned}
\partial_{c}^{2}\left(\rho^{2} \mathcal{L}_{1}(g)\right)= & 2 \rho^{\prime \prime} \rho \mathcal{L}_{1}(g)+4 \rho^{\prime} \rho \mathcal{L}_{1}\left(g^{\prime}\right)+2 \rho^{\prime} \rho^{\prime} \mathcal{L}_{1}(g)+4 \rho^{\prime} \rho \mathcal{L}_{2}(g)-2 \rho^{\prime} \mathcal{B}(g) \\
& +\rho^{2} \mathcal{L}_{1}\left(g^{\prime \prime}\right)+2 \rho^{2} \mathcal{L}_{2}\left(g^{\prime}\right)-\rho \mathcal{B}\left(g^{\prime}\right)+\rho^{2} \mathcal{L}_{3}(g)-\rho \mathcal{B}_{1}(g)-\partial_{c}(\rho \mathcal{B}(g)) .
\end{aligned}
$$

Proof. The lemma can be proved by using Lemma 11.1. We omit the details.

11.2. Boundedness of singular integral operators. Let $H$ be the Hilbert operator which is defined by

$$
H f(c)=\text { p.v. } \int \frac{f\left(c^{\prime}\right)}{c-c^{\prime}} d c^{\prime} .
$$

The maximal Hilbert transform $H^{*}$ is defined by

$$
H^{*} f(c)=\sup _{\epsilon>0}\left|H^{\epsilon} f(c)\right|, \quad H^{\epsilon} f(c)=\int_{\left|c-c^{\prime}\right| \geq \epsilon} \frac{f\left(c^{\prime}\right)}{c-c^{\prime}} d c^{\prime} .
$$

We denote

$$
\mathrm{H} f(c) \triangleq \chi_{D_{0}}(c) H\left(f \chi_{D_{0}}\right)(c) .
$$

Lemma 11.5. Suppose that $f \in H^{2}\left(D_{0}\right)$, then it holds that for any $p \in(1, \infty)$,

$$
\begin{aligned}
& \|\mathrm{H} f\|_{L^{p}} \leq C\|f\|_{L^{p}}, \\
& \|\rho \mathrm{H} f\|_{W^{1, p}} \leq C\|f\|_{W^{1, p}}, \\
& \left\|\rho^{2} \mathrm{H} f\right\|_{W^{2, p}} \leq C\|f\|_{W^{2, p}} .
\end{aligned}
$$

Proof. Thanks to

$$
\begin{aligned}
{[\rho, \mathrm{H}] f(c) } & =\int_{u(0)}^{u(1)} \frac{\rho(c)-\rho\left(c^{\prime}\right)}{c-c^{\prime}} f\left(c^{\prime}\right) d c^{\prime} \\
& =\int_{u(0)}^{u(1)}\left(u(0)+u(1)-c-c^{\prime}\right) f\left(c^{\prime}\right) d c^{\prime}
\end{aligned}
$$

we deduce that

$$
\left\|\partial_{c}[\rho, \mathrm{H}] f\right\|_{L^{p}}+\left\|\partial_{c}^{2}\left[\rho^{2}, \mathrm{H}\right] f\right\|_{L^{p}} \leq C\|f\|_{L^{p}}
$$


We have

$$
\begin{aligned}
\partial_{c}\left(\mathrm{p} . \mathrm{v} \cdot \int_{u(0)}^{u(1)} \frac{\rho\left(c^{\prime}\right) f\left(c^{\prime}\right)}{c-c^{\prime}} d c^{\prime}\right) & =\partial_{c}\left(\mathrm{p} . \mathrm{v} \cdot \int_{c-u(1)}^{c-u(0)} \frac{\rho(c-z) f(c-z)}{z} d z\right) \\
& =\text { p.v. } \int_{c-u(1)}^{c-u(0)} \frac{(\rho f)^{\prime}(c-z)}{z} d z=\mathrm{p} . \mathrm{v} \cdot \int_{u(0)}^{u(1)} \frac{(\rho f)^{\prime}\left(c^{\prime}\right)}{c-c^{\prime}} d c^{\prime},
\end{aligned}
$$

from which and $L^{p}$ boundedness of Hilbert transform, we infer that

$$
\begin{aligned}
& \| \partial_{c}\left(\text { p.v. } \int_{u(0)}^{u(1)} \frac{\rho\left(c^{\prime}\right) f\left(c^{\prime}\right)}{c-c^{\prime}} d c^{\prime}\right)\left\|_{L^{p}} \leq C\right\|(\rho f)^{\prime} \|_{L^{p}}, \\
& \| \partial_{c}^{2}\left(\text { p.v. } \int_{u(0)}^{u(1)} \frac{\rho\left(c^{\prime}\right)^{2} f\left(c^{\prime}\right)}{c-c^{\prime}} d c^{\prime}\right)\left\|_{L^{p}} \leq C_{0}\right\|\left(\rho^{2} f\right)^{\prime \prime} \|_{L^{p}},
\end{aligned}
$$

which along with (11.1) gives the lemma.

Lemma 11.6. There exists a constant $C$ independent of $\epsilon \in(0,(u(1)-u(0)) / 10)$ such that for any $p \in(1, \infty)$

$$
\begin{aligned}
& \left\|\sup _{\epsilon}\left|\int_{\left\{\left|c-c^{\prime}\right|>\epsilon\right\} \cap[u(0), u(1)]} \frac{\int_{c}^{c^{\prime}} f(z) d z}{\left(c-c^{\prime}\right)^{2}} d c^{\prime}\right|\right\|_{L^{p}} \leq C\|f\|_{L^{p}}, \\
& \| \text { p.v. } \int_{u(0)}^{u(1)} \frac{\int_{c}^{c^{\prime}} f(z) d z}{\left(c-c^{\prime}\right)^{2}} d c^{\prime}\left\|_{L^{p}} \leq C\right\| f \|_{L^{p}} .
\end{aligned}
$$

Proof. For $u(0) \leq c<u(0)+\epsilon$, we have

$$
\begin{aligned}
\int_{c+\epsilon}^{u(1)} \frac{\int_{c}^{c^{\prime}} f(z) d z}{\left(c-c^{\prime}\right)^{2}} d c^{\prime} & =\int_{c+\epsilon}^{u(1)}-\partial_{c^{\prime}}\left(\frac{1}{c^{\prime}-c}\right) \int_{c}^{c^{\prime}} f(z) d z d c^{\prime} \\
& =-\frac{1}{u(1)-c} \int_{c}^{u(1)} f(z) d z+\frac{1}{\epsilon} \int_{c}^{c+\epsilon} f(z) d z+\int_{c+\epsilon}^{u(1)} \frac{f(z)}{z-c} d z .
\end{aligned}
$$

For $u(0)+\epsilon \leq c \leq u(1)-\epsilon$, we have

$$
\begin{aligned}
& \int_{c+\epsilon}^{u(1)}+\int_{u(0)}^{c-\epsilon} \frac{\int_{c}^{c^{\prime}} f(z) d z}{\left(c^{\prime}-c\right)^{2}} d c^{\prime} \\
& =-\int_{c+\epsilon}^{u(1)}+\int_{u(0)}^{c-\epsilon} \partial_{c^{\prime}}\left(\frac{1}{c^{\prime}-c}\right) \int_{c}^{c^{\prime}} f(z) d z d c^{\prime} \\
& =-\frac{1}{u(1)-c} \int_{c}^{u(1)} f(z) d z+\frac{1}{\epsilon} \int_{c}^{c+\epsilon} f(z) d z+\int_{c+\epsilon}^{u(1)}+\int_{u(0)}^{c-\epsilon} \frac{f(z)}{z-c} d z \\
& -\frac{1}{\epsilon} \int_{c-\epsilon}^{c} f(z) d z+\frac{1}{c-u(0)} \int_{u(0)}^{c} f(z) d z .
\end{aligned}
$$

For $u(1)-\epsilon<c \leq u(1)$,

$$
\begin{aligned}
\int_{u(0)}^{c-\epsilon} \frac{\int_{c}^{c^{\prime}} f(z) d z}{\left(c^{\prime}-c\right)^{2}} d c^{\prime} & =\int_{u(0)}^{c-\epsilon}-\partial_{c^{\prime}}\left(\frac{1}{c^{\prime}-c}\right) \int_{c}^{c^{\prime}} f(z) d z d c^{\prime} \\
& =\frac{1}{c-u(0)} \int_{u(0)}^{c} f(z) d z-\frac{1}{\epsilon} \int_{c-\epsilon}^{c} f(z) d z+\int_{u(0)}^{c-\epsilon} \frac{f(z)}{z-c} d z .
\end{aligned}
$$


LINEAR INVISCID DAMPING FOR A CLASS OF MONOTONE SHEAR FLOW IN SOBOLEV SPACES 45

Thus, we obtain

$$
\begin{aligned}
\int_{\left\{\left|c-c^{\prime}\right|>\epsilon\right\} \cap[u(0), u(1)]} & \frac{\int_{c}^{c^{\prime}} f(z) d z}{\left(c-c^{\prime}\right)^{2}} d c^{\prime}=-\chi_{D_{0}}(c) H^{\epsilon}\left(f \chi_{D_{0}}\right)(c) \\
& +\left(\frac{1}{c-u(0)} \int_{u(0)}^{c} f(z) d z-\frac{1}{\epsilon} \int_{c-\epsilon}^{c} f(z) d z\right) \chi_{[\epsilon, u(1)]}(c) \\
& -\left(\frac{1}{u(1)-c} \int_{c}^{u(1)} f(z) d z-\frac{1}{\epsilon} \int_{c}^{c+\epsilon} f(z) d z\right) \chi_{[u(0), u(1)-\epsilon]}(c) .
\end{aligned}
$$

Taking $\epsilon \rightarrow 0+$ and using the fact that $\frac{1}{\epsilon} \int_{x-\epsilon}^{x} f(y) d y-\frac{1}{\epsilon} \int_{x}^{x+\epsilon} f(y) d y \rightarrow 0$, we deduce

$$
\begin{aligned}
\text { p.v. } \int_{u(0)}^{u(1)} \frac{\int_{c}^{c^{\prime}} f(z) d z}{\left(c-c^{\prime}\right)^{2}} d c^{\prime}= & -\chi_{D_{0}}(c) H\left(f \chi_{D_{0}}\right)(c) \\
& +\frac{1}{c-u(0)} \int_{u(0)}^{c} f(z) d z-\frac{1}{u(1)-c} \int_{c}^{u(1)} f(z) d z .
\end{aligned}
$$

With the above identities, the boundedness in $L^{p}$ follows from $L^{p}$ boundedness of Hilbert transform, maximal Hilbert transform and Hardy-Littlewood maximal function.

\subsection{Boundedness of multilinear singular integral operators.}

Lemma 11.7. There exists a constant $C$ independent of $\alpha$ such that for any $p \in(1, \infty)$,

$$
\left\|\mathbb{T}\left(f, g, \phi_{1}\left(u^{-1}, c\right), \frac{1}{\phi_{1}\left(u^{-1}, c\right)^{2}}\right)\right\|_{L^{p}} \leq C\left(\|f\|_{L^{\infty}}+\left\|f^{\prime}\right\|_{L^{\infty}}\right)\|g\|_{L^{p}}
$$

Proof. We write

$$
\begin{aligned}
& \mathbb{T}\left(f, g, \phi_{1}\left(u^{-1}, c\right), \frac{1}{\phi_{1}\left(u^{-1}, c\right)^{2}}\right)(c) \\
& =\int_{u(0)}^{u(1)} \frac{\int_{c}^{c^{\prime}} g(z)\left(\phi_{1}\left(u^{-1}(z), c\right)-1\right) d z}{\left(c^{\prime}-c\right)^{2} \phi_{1}\left(u^{-1}\left(c^{\prime}\right), c\right)^{2}} f\left(c^{\prime}\right) d c^{\prime} \\
& \quad+\int_{u(0)}^{u(1)} \frac{\int_{c}^{c^{\prime}} g(z) d z}{\left(c^{\prime}-c\right)^{2}} \frac{f\left(c^{\prime}\right)-f(c)}{\phi_{1}\left(u^{-1}\left(c^{\prime}\right), c\right)^{2}} d c^{\prime} \\
& \quad+f(c) \int_{\left\{\left|c^{\prime}-c\right| \leq \frac{1}{\alpha}\right\} \cap D_{0}} \frac{\int_{c}^{c^{\prime}} g(z) d z}{\left(c^{\prime}-c\right)^{2}} \frac{\left(\phi_{1}\left(u^{-1}\left(c^{\prime}\right), c\right)+1\right)\left(\phi_{1}\left(u^{-1}\left(c^{\prime}\right), c\right)-1\right)}{\phi_{1}\left(u^{-1}\left(c^{\prime}\right), c\right)^{2}} d c^{\prime} \\
& \quad+f(c) \int_{\left\{\left|c^{\prime}-c\right|>\frac{1}{\alpha}\right\} \cap D_{0}} \frac{\int_{c}^{c^{\prime}} g(z) d z}{\left(c^{\prime}-c\right)^{2} \phi_{1}\left(u^{-1}\left(c^{\prime}\right), c\right)^{2}} d c^{\prime} \\
& \quad+f(c) \text { p.v. } \int_{u(0)}^{u(1)} \frac{\int_{c}^{c^{\prime}} g(z) d z}{\left(c^{\prime}-c\right)^{2}} d c^{\prime}-f(c) \text { p.v. } \int_{\left\{\left|c^{\prime}-c\right|>\frac{1}{\alpha}\right\} \cap D_{0}} \frac{\int_{c}^{c^{\prime}} g(z) d z}{\left(c^{\prime}-c\right)^{2}} d c^{\prime} \\
& \triangleq I_{1}+\cdots I_{6} .
\end{aligned}
$$

Step 1. Estimate of $I_{1}$

We infer from Remark 5.5 that

$$
I_{1}=\alpha^{2} \int_{u(0)}^{u(1)} \frac{\int_{c}^{c^{\prime}} g(z)\left(u^{-1}(z)-u^{-1}(c)\right)^{2} \mathcal{T}\left(\phi_{1}\right)\left(u^{-1}(z), c\right) d z}{\left(c^{\prime}-c\right)^{2} \phi_{1}\left(u^{-1}\left(c^{\prime}\right), c\right)^{2}} f\left(c^{\prime}\right) d c^{\prime} .
$$


For $|z-c| \leq\left|c^{\prime}-c\right|$, we have $\left(u^{-1}(z)-u^{-1}(c)\right)^{2} \leq C\left(c^{\prime}-c\right)^{2}$. And by Remark 5.5,

$$
\mathcal{T}\left(\phi_{1}\right)\left(u^{-1}(z), c\right) \leq C \phi_{1}\left(u^{-1}(z), c\right) \leq C \phi_{1}\left(u^{-1}\left(c^{\prime}\right), c\right) .
$$

Then we infer that

$$
\left|I_{1}\right| \leq C \alpha^{2}\|f\|_{L^{\infty}} \int_{u(0)}^{u(1)} \int_{c}^{c^{\prime}}|g(z)| d z \frac{1}{\phi_{1}\left(u^{-1}\left(c^{\prime}\right), c\right)} d c^{\prime},
$$

from which and Proposition 5.1, it follows that

$$
\begin{aligned}
\left|I_{1}\right| & \leq C \alpha^{2}\|f\|_{L^{\infty}}\|g\|_{L^{\infty}} \int_{0}^{1} \frac{\left|y-y_{c}\right|}{\phi_{1}(y, c)} d y \\
& \leq C\left(\int_{0}^{\alpha\left(1-y_{c}\right)} \frac{y^{2}}{\sinh y} d y+\int_{0}^{\alpha y_{c}} \frac{y^{2}}{\sinh y} d y\right)\|f\|_{L^{\infty}}\|g\|_{L^{\infty}} \\
& \leq C\|f\|_{L^{\infty}}\|g\|_{L^{\infty}} .
\end{aligned}
$$

And by Proposition 5.1 again, we get

$$
\begin{aligned}
\left\|I_{1}\right\|_{L^{1}} \leq & C\|f\|_{L^{\infty}}\left(\int_{0}^{1} \int_{0}^{y_{c}} \alpha^{2} \int_{z}^{y_{c}}\left|g\left(u\left(y^{\prime \prime}\right)\right)\right| \frac{\alpha\left(z-y_{c}\right)}{\sinh \alpha\left(z-y_{c}\right)} d y^{\prime \prime} d z d y_{c}\right. \\
& \left.+\int_{0}^{1} \int_{y_{c}}^{1} \alpha^{2} \int_{y_{c}}^{z}\left|g\left(u\left(y^{\prime \prime}\right)\right)\right| \frac{\alpha\left(z-y_{c}\right)}{\sinh \alpha\left(z-y_{c}\right)} d y^{\prime \prime} d z d y_{c}\right) \\
\leq & C \alpha^{2}\|f\|_{L^{\infty}} \int_{0}^{1}\left|g\left(u\left(y^{\prime \prime}\right)\right)\right| \int_{0}^{y^{\prime \prime}} \int_{y^{\prime \prime}}^{1} \frac{\alpha\left(z-y_{c}\right)}{\sinh \alpha\left(z-y_{c}\right)} d y_{c} d z d y^{\prime \prime} \\
& +C \alpha^{2}\|f\|_{L^{\infty}} \int_{0}^{1}\left|g\left(u\left(y^{\prime \prime}\right)\right)\right| \int_{0}^{y^{\prime \prime}} \int_{y^{\prime \prime}}^{1} \frac{\alpha\left(z-y_{c}\right)}{\sinh \alpha\left(z-y_{c}\right)} d z d y_{c} d y^{\prime \prime} .
\end{aligned}
$$

Using the fact that

$$
\frac{z}{\sinh z} \leq 2 \frac{\sinh \frac{z}{2}}{\sinh z} \leq \frac{1}{\cosh \frac{z}{2}} \leq \frac{1}{e^{\frac{z}{2}}}, z>0
$$

we deduce that

$$
\begin{aligned}
\left|\alpha^{2} \int_{0}^{y} \int_{y}^{1} \frac{\alpha\left(z-y_{c}\right)}{\sinh \alpha\left(z-y_{c}\right)} d y_{c} d z\right| & \leq \int_{\alpha y}^{+\infty} \int_{0}^{\alpha y} e^{\frac{1}{2}\left(z-y_{c}\right)} d z d y_{c} \\
& \leq 2 \int_{\alpha y}^{+\infty} e^{\frac{1}{2} \alpha\left(y-y_{c}\right)}-e^{-\frac{1}{2} y_{c}} d y_{c} \leq 8
\end{aligned}
$$

This shows that

$$
\left\|I_{1}\right\|_{L^{1}} \leq C\|f\|_{L^{\infty}}\|g\|_{L^{1}} .
$$

Then by the interpolation, we get

$$
\left\|I_{1}\right\|_{L^{p}} \leq C\|f\|_{L^{\infty}}\|g\|_{L^{p}}
$$

Step 2. Estimate of $I_{2}$

Thanks to $\phi_{1}\left(u^{-1}(z), c\right)^{2} \geq 1$, we have

$$
\left|I_{2}\right| \leq C \int_{u(0)}^{u(1)} \frac{\int_{c}^{c^{\prime}}|g(z)| d z}{c^{\prime}-c} \frac{\left\|f^{\prime}\right\|_{L^{\infty}}}{\phi_{1}\left(u^{-1}\left(c^{\prime}\right), c\right)^{2}} d c^{\prime}
$$


LINEAR INVISCID DAMPING FOR A CLASS OF MONOTONE SHEAR FLOW IN SOBOLEV SPACES 47

$$
\leq C\left\|f^{\prime}\right\|_{L^{\infty}} \int_{u(0)}^{u(1)} \frac{\int_{c}^{c^{\prime}}|g(z)| d z}{c^{\prime}-c} d c^{\prime} \leq C\left\|f^{\prime}\right\|_{L^{\infty}}\|g\|_{L^{\infty}} .
$$

For $L^{1}$ estimate, we write

$$
\begin{aligned}
\int_{u(0)}^{u(1)} \frac{\int_{c}^{c^{\prime}}|g(z)| d z}{c^{\prime}-c} d c^{\prime} & =\int_{u(0)}^{c} \frac{\int_{c}^{c^{\prime}}|g(z)| d z}{c^{\prime}-c} d c^{\prime}+\int_{c}^{u(1)} \frac{\int_{c}^{c^{\prime}}|g(z)| d z}{c^{\prime}-c} d c^{\prime} \\
& =\int_{u(0)}^{c}|g(z)| \int_{u(0)}^{z} \frac{1}{c-c^{\prime}} d c^{\prime} d z+\int_{c}^{u(1)}|g(z)| \int_{z}^{u(1)} \frac{1}{c^{\prime}-c} d c^{\prime} d z \\
& =\int_{u(0)}^{c}|g(z)| \ln \frac{c-u(0)}{c-z} d z+\int_{c}^{u(1)}|g(z)| \ln \frac{u(1)-c}{z-c} d z,
\end{aligned}
$$

from which, it follows that

$$
\begin{aligned}
\left\|\int_{u(0)}^{u(1)} \frac{\int_{c}^{c^{\prime}}|g(z)| d z}{c^{\prime}-c} d c^{\prime}\right\|_{L^{1}} & \leq \int_{u(0)}^{u(1)} \int_{u(0)}^{c}|g(z)| \ln \frac{c-u(0)}{c-z} d z+\int_{c}^{u(1)}|g(z)| \ln \frac{u(1)-c}{z-c} d z d c \\
& \leq \int_{u(0)}^{u(1)}|g(z)| \int_{z}^{u(1)} \ln \frac{c-u(0)}{c-z} d c d z+\int_{u(0)}^{u(1)}|g(z)| \int_{u(0)}^{z} \ln \frac{u(1)-c}{z-c} d c d z \\
& \leq C\|g\|_{L^{1}} .
\end{aligned}
$$

This gives

$$
\left\|I_{2}\right\|_{L^{1}} \leq C\left\|f^{\prime}\right\|_{L^{\infty}}\|g\|_{L^{1}}
$$

By the interpolation, we get

$$
\left\|I_{2}\right\|_{L^{p}} \leq C\left\|f^{\prime}\right\|_{L^{\infty}}\|g\|_{L^{p}}
$$

Step 3. Estimate of $I_{3}$

By Lemma 5.4, $\left|\phi_{1}\left(u^{-1}\left(c^{\prime}\right), c\right)-1\right| \leq \alpha^{2}\left(c^{\prime}-c\right)^{2} \phi_{1}\left(u^{-1}\left(c^{\prime}\right), c\right)$. Then we have

$$
\begin{aligned}
\left|I_{3}\right| \leq & C \alpha^{2}\|f\|_{L^{\infty}} \int_{\left\{0 \leq c^{\prime}-c \leq \frac{1}{\alpha}\right\} \cap D_{0}} \int_{c}^{c^{\prime}}|g(z)| d z d c^{\prime} \\
& +C \alpha^{2}\|f\|_{L^{\infty}} \int_{\left\{0 \leq c-c^{\prime} \leq \frac{1}{\alpha}\right\} \cap D_{0}} \int_{c^{\prime}}^{c}|g(z)| d z d c^{\prime} \\
\leq & C\|f\|_{L^{\infty}} \alpha^{2} \int_{c}^{\min \left\{c+\frac{1}{\alpha}, u(1)\right\}} \int_{c}^{c^{\prime}}|g(z)| d z d c^{\prime} \\
& +C\|f\|_{L^{\infty}} \alpha^{2} \int_{\max \left\{c-\frac{1}{\alpha}, u(0)\right\}}^{c} \int_{c^{\prime}}^{c}|g(z)| d z d c^{\prime},
\end{aligned}
$$

from which, it follows that

$$
\begin{aligned}
\left\|I_{3}\right\|_{L^{\infty}} & \leq C\|f\|_{L^{\infty}}\left\|\alpha^{2} \int_{\left\{\left|c^{\prime}-c\right| \leq \frac{1}{\alpha}\right\} \cap D_{0}}\right\| g\left\|_{L^{\infty}}\left|c^{\prime}-c\right| d c^{\prime}\right\|_{L^{\infty}} \\
& \leq C\|f\|_{L^{\infty}}\|g\|_{L^{\infty}},
\end{aligned}
$$

and

$$
\left\|I_{3}\right\|_{L^{1}} \leq C \alpha^{2}\|f\|_{L^{\infty}}\left(\int_{u(0)}^{u(1)} \int_{c}^{\min \left\{c+\frac{1}{\alpha}, u(1)\right\}} \int_{c}^{c^{\prime}}|g(z)| d z d c^{\prime} d c\right.
$$




$$
\begin{aligned}
& \left.+\int_{u(0)}^{u(1)} \int_{\max \left\{c-\frac{1}{\alpha}, u(0)\right\}}^{c} \int_{c^{\prime}}^{c}|g(z)| d z d c^{\prime} d c\right) \\
\leq C \alpha^{2}\|f\|_{L^{\infty}} & \left(\int_{u(0)}^{u(1)}|g(z)| \int_{z-\frac{1}{\alpha}}^{z}\left(c+\frac{1}{\alpha}-z\right) d c d z\right. \\
& \left.+\int_{u(0)}^{u(1)}|g(z)| \int_{z}^{z+\frac{1}{\alpha}}\left(\frac{1}{\alpha}+z-c\right) d c d z\right) \\
\leq C\|f\|_{L^{\infty}}\|g\|_{L^{1}} &
\end{aligned}
$$

Thus by the interpolation, we get

$$
\left\|I_{3}\right\|_{L^{p}} \leq C\|f\|_{L^{\infty}}\|g\|_{L^{p}}
$$

Step 4. Estimate of $I_{4}$

We infer from Proposition 5.1 that

which gives

$$
\frac{1}{\phi_{1}\left(u^{-1}\left(c^{\prime}\right), c\right)} \geq C^{-1} \frac{\alpha\left(u^{-1}\left(c^{\prime}\right)-u^{-1}(c)\right)}{\sinh \alpha\left(u^{-1}\left(c^{\prime}\right)-u^{-1}(c)\right)},
$$

$$
\begin{aligned}
\left|I_{4}\right| & \leq C\|f\|_{L^{\infty}} \int_{\left|c^{\prime}-c\right| \geq \frac{1}{\alpha}}\|g\|_{L^{\infty}} \frac{\alpha^{2}\left|u^{-1}\left(c^{\prime}\right)-u^{-1}(c)\right|}{\sinh ^{2} \alpha\left(u^{-1}\left(c^{\prime}\right)-u^{-1}(c)\right)} d c^{\prime} \\
& \leq C\|f\|_{L^{\infty}}\|g\|_{L^{\infty}} \int_{1}^{\infty} \frac{z}{\sinh ^{2} z} d z \\
& \leq C\|f\|_{L^{\infty}}\|g\|_{L^{\infty}},
\end{aligned}
$$

and

$$
\begin{aligned}
\left\|I_{4}\right\|_{L^{1}} \leq & C \alpha^{2}\|f\|_{L^{\infty}} \int_{u(0)}^{u(1)} \int_{\left\{|z-c| \geq \frac{1}{\alpha}\right\} \cap\{z \geq c\} \cap[u(0), u(1)]} \int_{c}^{z}\left|g\left(c^{\prime}\right)\right| \frac{1}{\phi_{1}\left(u^{-1}(z), c\right)^{2}} d c^{\prime} d z d c \\
& +C \alpha^{2}\|f\|_{L^{\infty}} \int_{u(0)}^{u(1)} \int_{\left\{|z-c| \geq \frac{1}{\alpha}\right\} \cap\{z<c\} \cap[u(0), u(1)]} \int_{z}^{c}\left|g\left(c^{\prime}\right)\right| \frac{1}{\phi_{1}\left(u^{-1}(z), c\right)^{2}} d c^{\prime} d z d c .
\end{aligned}
$$

By a change of variable, the first term is bounded by

$$
\begin{aligned}
& C\|f\|_{L^{\infty}} \int_{0}^{1} \int_{\left\{\left|u\left(y^{\prime \prime}\right)-c\right| \geq \frac{1}{\alpha}\right\} \cap\left\{u\left(y^{\prime \prime}\right) \geq c\right\} \cap[0,1]} \int_{y_{c}}^{y^{\prime \prime}}\left|g\left(u\left(y^{\prime}\right)\right)\right| \frac{\alpha^{2}}{\phi_{1}\left(y^{\prime \prime}, c\right)^{2}} d y^{\prime} d y^{\prime \prime} d y_{c} \\
& \leq C\|f\|_{L^{\infty}} \int_{0}^{1}\left|g\left(u\left(y^{\prime}\right)\right)\right| \int_{0}^{y^{\prime}} \int_{y^{\prime}}^{1} \frac{\alpha^{2}}{\phi_{1}\left(y^{\prime \prime}, c\right)^{2}} d y^{\prime \prime} d y_{c} d y^{\prime} \\
& \leq C\|f\|_{L^{\infty}}\|g\|_{L^{1}},
\end{aligned}
$$

where we used the fact that $\phi_{1}\left(y^{\prime \prime}, c\right) \geq C^{-1} \frac{\sinh \alpha\left(y^{\prime \prime}-y_{c}\right)}{\alpha\left(y^{\prime \prime}-y_{c}\right)}$ so that

$$
\begin{aligned}
\left|\int_{0}^{y^{\prime}} \int_{y^{\prime}}^{1} \frac{\alpha^{2}}{\phi_{1}^{2}\left(y^{\prime \prime}, c\right)} d y^{\prime \prime} d y_{c}\right| & \leq C \int_{0}^{y^{\prime}} \int_{y^{\prime}}^{1} \frac{\alpha^{4}\left(y^{\prime \prime}-y_{c}\right)^{2}}{\sinh \alpha\left(y^{\prime \prime}-y_{c}\right)^{2}} d y^{\prime \prime} d y_{c} \\
& \leq C \int_{0}^{\alpha y^{\prime}} \int_{\alpha y^{\prime}}^{+\infty} e^{-1 / 2\left(y-y_{c}\right)} d y d y_{c} \leq C .
\end{aligned}
$$

The estimate of the second term is the same. This gives

$$
\left\|I_{4}\right\|_{L^{1}} \leq C\|f\|_{L^{\infty}}\|g\|_{L^{1}} .
$$


Thus, the interpolation gives

$$
\left\|I_{4}\right\|_{L^{p}} \leq C\|f\|_{L^{\infty}}\|g\|_{L^{p}}
$$

Step 5. Estimates of $I_{5}$ and $I_{6}$

Lemma 11.6 ensures that

$$
\left\|I_{5}\right\|_{L^{p}}+\left\|I_{6}\right\|_{L^{p}} \leq C\|f\|_{L^{\infty}}\|g\|_{L^{p}} .
$$

Putting the estimates in Step 1-Step 5 together, we conclude the lemma.

Lemma 11.8. There exists a constant $C$ independent of $\alpha$ such that for any $p \in(1, \infty)$,

$$
\begin{aligned}
& \left\|\mathbb{B}_{0}\left(f, \rho g, F, \partial_{c} G\right)\right\|_{L^{p}}+\left\|\mathbb{B}_{1}\left(f, \rho g, F, \partial_{c} G\right)\right\|_{L^{p}} \\
& +\left\|\mathbb{B}_{0}\left(f, g,\left(\partial_{z}+\partial_{c}\right) F, G\right)\right\|_{L^{p}}+\left\|\mathbb{B}_{1}\left(f, g,\left(\partial_{z}+\partial_{c}\right) F, G\right)\right\|_{L^{p}} \leq C\|f\|_{L^{\infty}}\|g\|_{L^{p}},
\end{aligned}
$$

where $F(z, c)=\phi_{1}\left(u^{-1}(z), c\right)$ and $G(z, c)=\frac{1}{\phi_{1}\left(u^{-1}(z), c\right)^{2}}$. We also have

$$
\|\mathcal{B}(\rho g)\|_{L^{p}} \leq \frac{1}{\alpha}\|g\|_{L^{p}} .
$$

Proof. We only prove the estimate of $\mathbb{B}_{0}$, the estimate of $\mathbb{B}_{1}$ is similar. We get by Proposition 5.1 that

$$
\left|\mathbb{B}_{0}\left(f, \rho g, F, \partial_{c} G\right)(c)\right| \leq C\|f\|_{L^{\infty}} \frac{\alpha}{\phi_{1}(0, c)} \int_{u(0)}^{c}|g(z)| d z,
$$

from which, it follows that

$$
\begin{aligned}
\left\|\mathbb{B}_{0}\left(f, \rho g, F, \partial_{c} G\right)\right\|_{L^{\infty}} & \leq C \sup _{y \in[0,1]} \frac{\alpha^{2} y^{2}}{\sinh \alpha y}\|f\|_{L^{\infty}}\|g\|_{L^{\infty}} \\
& \leq C\|f\|_{L^{\infty}}\|g\|_{L^{\infty}}, \\
\left\|\mathbb{B}_{0}\left(f, \rho g, F, \partial_{C} G\right)\right\|_{L^{1}} & \leq C\|f\|_{L^{\infty}} \int_{0}^{1}|g(z)| d z \int_{0}^{1} \frac{\alpha^{2} y}{\sinh \alpha y} d y \\
& \leq\|f\|_{L^{\infty}}\|g\|_{L^{1}} .
\end{aligned}
$$

We get by Proposition 5.6 that

$$
\left|\mathbb{B}_{0}\left(f, g,\left(\partial_{z}+\partial_{c}\right) F, G\right)(c)\right| \leq C\|f\|_{L^{\infty}} \frac{\alpha^{2}(c-u(0))}{\phi_{1}(0, c)} \int_{u(0)}^{c}|g(z)| d z,
$$

which implies that

$$
\begin{aligned}
& \left\|\mathbb{B}_{0}\left(f, g,\left(\partial_{z}+\partial_{c}\right) F, G\right)\right\|_{L^{\infty}} \leq\|f\|_{L^{\infty}}\|g\|_{L^{\infty}}, \\
& \left\|\mathbb{B}_{0}\left(f, g,\left(\partial_{z}+\partial_{c}\right) F, G\right)\right\|_{L^{1}} \leq C\|f\|_{L^{\infty}}\|g\|_{L^{1}} .
\end{aligned}
$$

Then $L^{p}$ estimate follows by the interpolation.

Notice that

$$
|\alpha \mathcal{B}(\rho g)| \leq C \frac{\alpha}{\phi_{1}(0, c)} \int_{u(0)}^{c}|g(z)| d z+C \frac{\alpha}{\phi_{1}(1, c)} \int_{c}^{u(1)}|g(z)| d z .
$$

Then $L^{p}$ estimate of $\mathcal{B}(\rho g)$ can be deduced in a similar way. 
Lemma 11.9. There exists a constant $C$ independent of $\alpha$ such that for any $p \in(1, \infty)$,

$$
\|\mathcal{B}(g)\|_{L^{p}}+\left\|\partial_{c}(\rho \mathcal{B}(g))\right\|_{L^{p}}+\left\|\rho^{-1} \mathcal{B}(\rho g)\right\|_{L^{p}}+\left\|\mathcal{B}_{1}(g)\right\|_{L^{p}} \leq C\|g\|_{L^{p}}
$$

If $g(u(0))=g(u(1))=0$, then we have

$$
\left\|\rho \mathcal{B}\left(g^{\prime}\right)\right\|_{L^{p}} \leq C\|g\|_{L^{p}}
$$

Proof. The estimate of $\|\mathcal{B}(g)\|_{L^{p}}$ follows from $L^{p}$ boundedness of Hardy-Littlewood maximal function.

Step 1. Estimate of $\left\|\partial_{c}(\rho \mathcal{B}(g))\right\|_{L^{p}}$

It suffices to consider $\mathbb{B}_{0}$. By the definition of $\mathbb{B}_{0}$, we have

$$
\begin{aligned}
& \partial_{c}\left(\rho(c) \mathbb{B}_{0}\left(f, g, \phi_{1}\left(u^{-1}(\cdot), c\right), \frac{1}{\phi_{1}(0, c)^{2}}\right)\right) \\
& =2(c-u(1)) f(u(0)) \int_{u(0)}^{c} g(z) \frac{\phi_{1}\left(u^{-1}(z), c\right)}{\phi_{1}(0, c)^{2}} d z+\frac{(u(1)-c)^{2} f(u(0)) g(c)}{\phi_{1}(0, c)^{2}} \\
& \quad+(u(1)-c)^{2} f(u(0)) \int_{u(0)}^{c} g(z) \partial_{c}\left(\frac{\phi_{1}\left(u^{-1}(z), c\right)}{\phi_{1}(0, c)^{2}}\right) d z .
\end{aligned}
$$

The $L^{p}$ estimate of the first two terms are obvious. So we only consider the last term, which is bounded by

$$
C\|f\|_{L^{\infty}} \int_{0}^{y_{c}}|g(u(y))| \frac{\alpha}{\phi_{1}(0, c)} d y \leq C\|f\|_{L^{\infty}} \frac{\alpha^{2} y_{c}}{\sinh \alpha y_{c}} \int_{0}^{y_{c}}|g(u(y))| d y,
$$

where we used Proposition 5.1. Thus, $L^{\infty}$ norm of the last term is bounded by

$$
C \sup _{y \in[0,1]} \frac{\alpha^{2} y^{2}}{\sinh \alpha y}\|g\|_{L^{\infty}}\|f\|_{L^{\infty}}
$$

while $L^{1}$ norm is bounded by

$$
C\|f\|_{L^{\infty}} \int_{0}^{1}|g(u(y))| \int_{y_{c}}^{1} \frac{\alpha^{2} y_{c}}{\sinh \alpha y_{c}} d y_{c} d y \leq C\|f\|_{L^{\infty}}\|g\|_{L^{1}} .
$$

This implies by the interpolation that

$$
\left\|\partial_{c}(\rho \mathcal{B}(g))\right\|_{L^{p}} \leq C\|g\|_{L^{p}}
$$

Step 2. Estimate of $\left\|\rho^{-1} \mathcal{B}(\rho g)\right\|_{L^{p}}$

Using the fact that

$$
\phi_{1}\left(u^{-1}(z), c\right) \leq \phi_{1}\left(u^{-1}\left(c^{\prime}\right), c\right) \text { for }|z-c| \leq\left|c^{\prime}-c\right|,
$$

we deduce that

$$
\begin{aligned}
\left|\rho^{-1} \mathcal{B}(\rho g)(c)\right| \leq & \frac{C}{(c-u(0))^{2}} \int_{u(0)}^{c} g(z)(z-u(0))(u(1)-z) d z \\
& +\frac{C}{(u(1)-c)^{2}} \int_{c}^{u(1)} g(z)(z-u(0))(u(1)-z) d z \\
\leq & \frac{C}{c-u(0)} \int_{u(0)}^{c} g(z) d z+\frac{C}{u(1)-c} \int_{c}^{u(1)} g(z) d z
\end{aligned}
$$

from which and $L^{p}$ boundedness of Hardy-Littlewood maximal function, we infer that

$$
\left\|\rho^{-1} \mathcal{B}(\rho g)\right\|_{L^{p}} \leq C\|g\|_{L^{p}}
$$


Step 3. Estimate of $\left\|\mathcal{B}_{1}(g)\right\|_{L^{p}}$

By Proposition 5.6, we have

$$
\begin{aligned}
& \left|\left(\partial_{z}+\partial_{c}\right) \phi_{1}\left(u^{-1}(z), c\right)\right| \leq C_{0} \alpha^{2}(z-c)^{2} \phi_{1}\left(u^{-1}(z), c\right), \\
& \left|\left(\partial_{z}+\partial_{c}\right)\left(\frac{1}{\phi_{1}\left(u^{-1}(z), c\right)^{2}}\right)\right| \leq C_{0} \alpha^{2}(z-c)^{2} \frac{1}{\phi_{1}\left(u^{-1}(z), c\right)^{2}},
\end{aligned}
$$

which along with (11.3) gives So we have

$$
\mathcal{B}_{1}(g) \leq \frac{C}{c-u(0)} \int_{u(0)}^{c}|\varphi(y)| d y+\frac{C}{u(1)-c} \int_{c}^{u(1)}|\varphi(y)| d y,
$$

from which and $L^{p}$ boundedness of Hardy-Littlewood maximal function, we infer that

$$
\left\|\mathcal{B}_{1}(g)\right\|_{L^{p}} \leq C\|g\|_{L^{p}} .
$$

Step 4. Estimate of $\left\|\rho \mathcal{B}\left(g^{\prime}\right)\right\|_{L^{2}}$

Using the boundary condition of $g$, we get

$$
\begin{aligned}
\rho(c) \mathcal{B}\left(g^{\prime}\right)= & (u(1)-c)^{2}\left(u^{-1}\right)^{\prime}(u(0)) \int_{u(0)}^{c} g^{\prime}(z) \frac{\phi_{1}\left(u^{-1}(z), c\right)}{\phi_{1}(0, c)^{2}} d z \\
& +(c-u(0))^{2}\left(u^{-1}\right)^{\prime}(u(1)) \int_{c}^{u(1)} g^{\prime}(z) \frac{\phi_{1}\left(u^{-1}(z), c\right)}{\phi_{1}(1, c)^{2}} d z \\
= & -(u(1)-c)^{2}\left(u^{-1}\right)^{\prime}(u(0)) \int_{u(0)}^{c} g(z) \frac{\partial_{z} \phi_{1}\left(u^{-1}(z), c\right)}{\phi_{1}(0, c)^{2}} d z \\
& -(c-u(0))^{2}\left(u^{-1}\right)^{\prime}(u(1)) \int_{c}^{u(1)} g(z) \frac{\partial_{z} \phi_{1}\left(u^{-1}(z), c\right)}{\phi_{1}(1, c)^{2}} d z \\
& +(u(1)-c)^{2}\left(u^{-1}\right)^{\prime}(u(0)) g(c) \frac{1}{\phi_{1}(0, c)^{2}} \\
& -(c-u(0))^{2}\left(u^{-1}\right)^{\prime}(u(1)) g(c) \frac{1}{\phi_{1}(1, c)^{2}} .
\end{aligned}
$$

Then by Proposition 5.1, we obtain

$\left|\rho(c) \mathcal{B}\left(g^{\prime}\right)(c)\right| \leq C|g(c)|+C \int_{0}^{y_{c}}|g(u(z))| \frac{\alpha \cosh \alpha\left(z-y_{c}\right)}{\phi_{1}(0, c)^{2}} d z+C \int_{y_{c}}^{1}|g(u(z))| \frac{\alpha \cosh \alpha\left(z-y_{c}\right)}{\phi_{1}(1, c)^{2}} d z$.

with $c=u\left(y_{c}\right)$. Thus, we have

$$
\begin{aligned}
\left\|\rho \mathcal{B}\left(g^{\prime}\right)\right\|_{L^{\infty}} & \leq C\|g\|_{L^{\infty}}+C\|g\|_{L^{\infty}} \sup _{c \in D_{0}}\left(\frac{\sinh \alpha y_{c}}{\phi_{1}(0, c)^{2}}+\frac{\sinh \alpha\left(1-y_{c}\right)}{\phi_{1}(1, c)^{2}}\right) \\
& \leq C\|g\|_{L^{\infty}} .
\end{aligned}
$$

On the other hand, we have

$$
\begin{aligned}
\left\|\rho(c) \mathcal{B}\left(g^{\prime}\right)\right\|_{L^{1}} \leq & C\|g\|_{L^{1}}+C \int_{0}^{1} \int_{0}^{y_{c}}|g(z)| \frac{\alpha \cosh \alpha\left(z-y_{c}\right)}{\phi_{1}(0, c)^{2}} d z d y_{c} \\
& +C \int_{0}^{1} \int_{y_{c}}^{1}|g(z)| \frac{\alpha \cosh \alpha\left(z-y_{c}\right)}{\phi_{1}(1, c)^{2}} d z d y_{c} \\
\leq & C\|g\|_{L^{1}}+C\|g\|_{L^{1}} \int_{0}^{\infty} \frac{z^{2} \cosh z}{\sinh ^{2}(z)} d z
\end{aligned}
$$




$$
\leq C\|g\|_{L^{1}}
$$

By the interpolation, we get

$$
\left\|\rho(c) \mathcal{B}\left(g^{\prime}\right)\right\|_{L^{p}} \leq C\|g\|_{L^{p}}
$$

The proof is completed.

Lemma 11.10. There exists a constant $C$ independent of $\alpha$ such that for any $p \in(1, \infty)$,

$$
\left\|\mathcal{L}_{1}(g)\right\|_{L^{p}}+\left\|\mathcal{L}_{2}(g)\right\|_{L^{p}}+\left\|\mathcal{L}_{3}(g)\right\|_{L^{p}} \leq C\|g\|_{L^{p}} .
$$

Proof. The estimate of $\left\|\mathcal{L}_{1}(g)\right\|_{L^{2}}$ follows from Lemma 11.7. Now we consider $\mathcal{L}_{2}(g)$. It suffices to consider the first two terms in the definition of $\mathcal{L}_{2}(g)$. By Proposition 5.6, both terms are bounded by

$$
C \alpha^{2} \int_{u(0)}^{u(1)}\left|\int_{c}^{c^{\prime}}\right| g(z)|d z| \frac{1}{\phi_{1}\left(u^{-1}\left(c^{\prime}\right), c\right)} d c^{\prime}
$$

With this, the proof is the same as $I_{1}$ in the proof of Lemma 11.7.

Next we consider $\mathcal{L}_{3}(g)$. It suffice to estimate the following terms

$$
\begin{aligned}
& \mathbb{T}\left(\left(u^{-1}\right)^{\prime}, g,\left(\partial_{z}+\partial_{c}\right)^{2} \phi_{1}\left(u^{-1}, c\right), \frac{1}{\phi_{1}\left(u^{-1}, c\right)^{2}}\right)(c) \\
& \quad+\mathbb{T}\left(\left(u^{-1}\right)^{\prime}, g, \phi_{1}\left(u^{-1}, c\right),\left(\partial_{z}+\partial_{c}\right)^{2}\left(\frac{1}{\phi_{1}\left(u^{-1}, c\right)^{2}}\right)\right)(c) \triangleq \mathcal{L}_{2}^{1}(g) .
\end{aligned}
$$

By Proposition 5.6, we have

$$
\left|\left(\partial_{z}+\partial_{c}\right)^{2} \phi_{1}\left(u^{-1}(z), c\right)\right| \leq C \alpha^{2}\left(u^{-1}(z)-u^{-1}(c)\right)^{2} \cosh \alpha\left(u^{-1}(z)-u^{-1}(c)\right),
$$

and

$$
\left|\left(\partial_{z}+\partial_{c}\right)\left(\frac{1}{\phi_{1}^{2}\left(u^{-1}(z), c\right)}\right)\right| \leq C \alpha^{2}\left(u^{-1}(z)-u^{-1}(c)\right)^{2} \frac{\cosh \alpha\left(u^{-1}(z)-u^{-1}(c)\right)}{\phi_{1}^{3}\left(u^{-1}(z), c\right)},
$$

which imply

$$
\left|\mathcal{L}_{2}^{1}(g)(c)\right| \leq C \alpha^{2} \int_{u(0)}^{u(1)}\left|\int_{c}^{c^{\prime}}\right| g(z)|d z| \frac{\cosh \alpha\left(u^{-1}\left(c^{\prime}\right)-u^{-1}(c)\right)}{\phi_{1}^{2}\left(u^{-1}\left(c^{\prime}\right), c\right)} d c^{\prime} .
$$

Thus, we obtain

$$
\left\|\mathcal{L}_{2}^{1}(g)\right\|_{L^{\infty}} \leq C \int_{0}^{\infty} \frac{z^{3} \cosh z}{\sinh ^{2} z} d z\|g\|_{L^{\infty}} \leq C\|g\|_{L^{\infty}}
$$

and

$$
\begin{aligned}
\left\|\mathcal{L}_{2}^{1}(g)\right\|_{L^{1}} \leq & C \alpha^{2} \int_{0}^{1}|g(y)| \int_{0}^{y} \int_{y}^{1} \frac{\alpha^{2}\left(z-y_{c}\right)^{2} \cosh \alpha\left(z-y_{c}\right)}{\sinh ^{2} \alpha\left(z-y_{c}\right)} d y_{c} d z d y \\
& +C \alpha^{2} \int_{0}^{1}|g(y)| \int_{0}^{y} \int_{y}^{1} \frac{\alpha^{2}\left(z-y_{c}\right)^{2} \cosh \alpha\left(z-y_{c}\right)}{\sinh ^{2} \alpha\left(z-y_{c}\right)} d z d y_{c} d y \\
\leq & C\|g\|_{L^{1}}
\end{aligned}
$$

where we used $\frac{z^{2} \cosh z}{\sinh ^{2} z} \leq \frac{C_{0}}{e^{z / 2}}$ for $z \geq 0$. By the interpolation, we get

$$
\left\|\mathcal{L}_{2}^{1}(g)\right\|_{L^{p}} \leq C\|g\|_{L^{p}} .
$$

This completes the proof of the lemma. 
LINEAR INVISCID DAMPING FOR A CLASS OF MONOTONE SHEAR FLOW IN SOBOLEV SPACES 53

Lemma 11.11. There exists a constant $C$ independent of $\alpha$ such that for any $p \in(1, \infty)$,

$$
\left\|\left[\mathcal{L}_{1}, \rho\right]\left(g^{\prime}\right)\right\|_{L^{p}}+\left\|\partial_{c}\left[\mathcal{L}_{1}, \rho\right](g)\right\|_{L^{p}} \leq C\|g\|_{L^{p}}
$$

Proof. We have

$$
\begin{aligned}
\partial_{c}\left[\mathcal{L}_{1}, \rho\right] g= & \mathcal{L}_{1}\left((\rho g)^{\prime}\right)+\mathcal{L}_{2}(\rho g)-\frac{1}{\rho} \mathcal{B}(\rho g) \\
& -\rho^{\prime} \mathcal{L}_{1}(g)-\rho \mathcal{L}_{1}\left(g^{\prime}\right)-\rho \mathcal{L}_{2}(g)+\mathcal{B}(g) \\
= & {\left[\mathcal{L}_{1}, \rho\right]\left(g^{\prime}\right)+\mathcal{L}_{1}\left(\rho^{\prime} g\right)+\mathcal{L}_{2}(\rho g)-\frac{1}{\rho} \mathcal{B}(\rho g) } \\
& -\rho^{\prime} \mathcal{L}_{1}(g)-\rho \mathcal{L}_{2}(g)+\mathcal{B}(g) .
\end{aligned}
$$

Thus, by Lemma 11.9 and Lemma 11.10, it suffices to consider $\left[\mathcal{L}_{1}, \rho\right]\left(g^{\prime}\right)$.

Since

$$
\begin{aligned}
{\left[\mathcal{L}_{1}, \rho\right]\left(g^{\prime}\right)(c)=} & \int_{u(0)}^{u(1)} \frac{\int_{c}^{c^{\prime}}(\rho(z)-\rho(c)) g^{\prime}(z) \phi_{1}\left(u^{-1}(z), c\right) d z}{\left(c^{\prime}-c\right)^{2}} \frac{\left(u^{-1}\right)^{\prime}\left(c^{\prime}\right)}{\phi_{1}\left(u^{-1}\left(c^{\prime}\right), c\right)^{2}} d c^{\prime} \\
= & p . v \cdot \int_{u(0)}^{u(1)} \frac{g\left(c^{\prime}\right)}{c^{\prime}-c} \frac{\left(u(0)+u(1)-c^{\prime}-c\right)\left(u^{-1}\right)^{\prime}\left(c^{\prime}\right)}{\phi_{1}\left(u^{-1}\left(c^{\prime}\right), c\right)} d c^{\prime} \\
& -\int_{u(0)}^{u(1)} \frac{\int_{c}^{c^{\prime}}(\rho(z)-\rho(c)) g(z) \partial_{z} \phi_{1}\left(u^{-1}(z), c\right) d z}{\left(c^{\prime}-c\right)^{2}} \frac{\left(u^{-1}\right)^{\prime}\left(c^{\prime}\right)}{\phi_{1}\left(u^{-1}\left(c^{\prime}\right), c\right)^{2}} d c^{\prime}-\mathcal{L}_{1}\left(\rho^{\prime} g\right) \\
\triangleq & \Theta_{1}+\Theta_{2}-\mathcal{L}_{1}\left(\rho^{\prime} g\right) .
\end{aligned}
$$

Let $f\left(c^{\prime}, c\right)=\left(u(0)+u(1)-c^{\prime}-c\right)\left(u^{-1}\right)^{\prime}\left(c^{\prime}\right)$. $\Theta_{1}$ can be rewritten as

$$
\begin{aligned}
& \text { p.v. } \int_{u(0)}^{u(1)} \frac{g\left(c^{\prime}\right)}{c^{\prime}-c} \frac{\left(u(0)+u(1)-c^{\prime}-c\right)\left(u^{-1}\right)^{\prime}\left(c^{\prime}\right)}{\phi_{1}\left(u^{-1}\left(c^{\prime}\right), c\right)} d c^{\prime} \\
& =(u(0)+u(1)-2 c)\left(u^{-1}\right)^{\prime}(c) \text { p.v. } \int_{u(0)}^{u(1)} \frac{g\left(c^{\prime}\right)}{c^{\prime}-c} d c^{\prime} \\
& \quad-(u(0)+u(1)-2 c)\left(u^{-1}\right)^{\prime}(c) \text { p.v. } \int_{D_{0} \cap\left\{\left|c^{\prime}-c\right|>\frac{1}{\alpha}\right\}} \frac{g\left(c^{\prime}\right)}{c^{\prime}-c} d c^{\prime} \\
& +\int_{u(0)}^{u(1)} \frac{g\left(c^{\prime}\right)}{c^{\prime}-c} \frac{f\left(c^{\prime}, c\right)-f(c, c)}{\phi_{1}\left(u^{-1}\left(c^{\prime}\right), c\right)} d c^{\prime} \\
& +\int_{D_{0} \cap\left\{\left|c^{\prime}-c\right|>\frac{1}{\alpha}\right\}} \frac{g\left(c^{\prime}\right)}{c^{\prime}-c} \frac{f(c, c)}{\phi_{1}\left(u^{-1}\left(c^{\prime}\right), c\right)} d c^{\prime} \\
& \quad-f(c, c) \int_{D_{0} \cap\left\{\left|c^{\prime}-c\right| \leq \frac{1}{\alpha}\right\}} \frac{g\left(c^{\prime}\right)}{c^{\prime}-c} \frac{\phi_{1}\left(u^{-1}\left(c^{\prime}\right), c\right)-1}{\phi_{1}\left(u^{-1}\left(c^{\prime}\right), c\right)} d c^{\prime} .
\end{aligned}
$$

The $L^{p}$ boundedness of the first two terms follows from $L^{p}$ boundedness of Hilbert transform. The third term is trivial since $\left|f\left(c^{\prime}, c\right)-f(c, c)\right| \leq C\left|c^{\prime}-c\right|$. The fourth term is bounded by

$$
C \int_{[0,1] \cap\left\{\left|x-x_{0}\right|>\frac{c_{0}}{\alpha}\right\}}|g(u(y))| \frac{\alpha^{2}\left|y-y_{c}\right|}{\sinh \alpha\left(y-y_{c}\right)} d y \text {. }
$$


Thanks to $K_{1}(z)=\alpha \frac{\alpha z}{\sinh \alpha z} \chi_{\left[c_{0},+\infty\right]}(\alpha z) \in L^{1}$, we get

$$
\begin{aligned}
\left\|\int_{D_{0} \cap\left\{\left|c^{\prime}-c\right|>\frac{1}{\alpha}\right\}} \frac{g\left(c^{\prime}\right)}{c^{\prime}-c} \frac{f(c, c)}{\phi_{1}\left(u^{-1}\left(c^{\prime}\right), c\right)} d c^{\prime}\right\|_{L^{p}} & \leq C\left\||g \circ u| * K_{1}\right\|_{L^{p}} \\
& \leq C\|g\|_{L^{p}} .
\end{aligned}
$$

The last term is bounded by

$$
C \int_{D_{0} \cap\left\{\left|c^{\prime}-c\right| \leq \frac{1}{\alpha}\right\}} g\left(c^{\prime}\right) \alpha^{2}\left|c^{\prime}-c\right| d c^{\prime}
$$

Thanks to $K_{2}(z)=\alpha^{2} z \chi_{[0,1]}(\alpha z) \in L^{1}$, we get

$$
\begin{aligned}
\left\|\int_{D_{0} \cap\left\{\left|c^{\prime}-c\right| \leq \frac{1}{\alpha}\right\}} \frac{g\left(c^{\prime}\right)}{c^{\prime}-c} \frac{\phi_{1}\left(u^{-1}\left(c^{\prime}\right), c\right)-1}{\phi_{1}\left(u^{-1}\left(c^{\prime}\right), c\right)} d c^{\prime}\right\|_{L^{p}} & \leq C_{0}\left\||g| * K_{2}\right\|_{L^{p}} \\
& \leq C_{0}\|g\|_{L^{p}} .
\end{aligned}
$$

This shows that

$$
\left\|\Theta_{1}\right\|_{L^{p}} \leq C\|g\|_{L^{p}}
$$

For $\Theta_{2}$ term, we have

$$
\left|\Theta_{2}(c)\right| \leq C \alpha \int_{0}^{1} \frac{\int_{y_{c}}^{y}|g(u(z))| d z}{y-y_{c}} \frac{\alpha\left(y-y_{c}\right)}{\sinh \alpha\left(y-y_{c}\right)} d y,
$$

from which, it follows that

$$
\begin{aligned}
\left\|\Theta_{2}(c)\right\|_{L^{\infty}} & \leq C \alpha \int_{0}^{1} \frac{\int_{y_{c}}^{y}\|g\|_{L^{\infty}} d z}{y-y_{c}} \frac{\alpha\left(y-y_{c}\right)}{\sinh \alpha\left(y-y_{c}\right)} d y \\
& \leq C \alpha\|g\|_{L^{\infty}} \int_{0}^{1} \frac{\alpha\left(y-y_{c}\right)}{\sinh \alpha\left(y-y_{c}\right)} d y \\
& \leq C\|g\|_{L^{\infty}}\left\|\frac{z}{\sinh z}\right\|_{L^{1}} \leq C\|g\|_{L^{\infty}},
\end{aligned}
$$

and

$$
\begin{aligned}
\left\|\Theta_{2}(c)\right\|_{L^{1}} & \leq C \int_{0}^{1} \alpha \int_{0}^{1} \frac{\int_{y_{c}}^{y}|g(u(z))| d z}{y-y_{c}} \frac{\alpha\left(y-y_{c}\right)}{\sinh \alpha\left(y-y_{c}\right)} d y d y_{c} \\
& \leq C \int_{0}^{1}|g(u(z))| d z\left\|\int_{0}^{z} \int_{z}^{1} \frac{\alpha^{2}}{\sinh \alpha\left(y_{c}-y\right)} d y_{c} d y\right\|_{L^{\infty}} \leq C\|g\|_{L^{1}} .
\end{aligned}
$$

By the interpolation, we get

$$
\left\|\Theta_{2}(c)\right\|_{L^{p}} \leq C\|g\|_{L^{p}}
$$

Thus, we obtain

$$
\left\|\left[\mathcal{L}_{1}, \rho\right]\left(g^{\prime}\right)\right\|_{L^{p}} \leq\left\|\Theta_{1}(c)\right\|_{L^{p}}+\left\|\Theta_{2}(c)\right\|_{L^{p}}+\left\|\mathcal{L}_{1}\left(\rho^{\prime} g\right)\right\|_{L^{p}} \leq C\|g\|_{L^{p}} .
$$

This completes the proof of the lemma. 


\section{ACKNOWLEDGEMENT}

Z. Zhang thanks Professor Zhiwu Lin for introducing this question to him and many profitable discussions. This work was done when W. Zhao was visiting School of Mathematical Science, Peking University. He appreciates the hospitality from PKU. Z. Zhang is partially supported by NSF of China under Grant 11371037 and 11425103. W. Zhao is partially supported by NSF of China under Grant 11571306.

\section{REFERENCES}

[1] V. I. Arnold, On an apriori estimate in the theory of hydrodynamical stability, Amer. Math. Soc. Transl., 79(1969), 267-269.

[2] C. Bardos, Y. Guo and W. Strauss, Stable and unstable ideal plane flows, Chinese Ann. Math. Ser. B, 23(2002), 149-164.

[3] J. Bedrossian and N. Masmoudi, Inviscid damping and the asymptotic stability of planar shear flows in the 2D Euler equations, arXiv:1306.5028.

[4] J. Bedrossian, N. Masmoudi and C. Mouhot, Landau damping: paraproducts and Gevrey regularity, arXiv:1311.2870.

[5] F. Bouchet and H. Morita, Large time behavior and asymptotic stability of the 2D Euler and linearized Euler equations, Physica D, 239(2010), 948-966.

[6] J.-Y. Chemin, Perfect Incompressible Fluids, Oxford Lecture Ser. Math. Appl. 14, The Clarendon Press, Oxford University Press, New York, 1998.

[7] K. M. Case, Stability of inviscid plane Couette flow, Phys. Fluids, 3(1960), 143-148.

[8] S. A. Denisov, Infinite superlinear growth of the gradient for the twodimensional Euler equation, Discrete Contin. Dyn. Syst., 23(2009), 755-764.

[9] P. G. Drazin and W. H. Reid, Hydrodynamics stability, Cambridge Monographs on Mech. and Applied Math., Cambridge University Press, Cambridge, 1981.

[10] E. Grenier, On the nonlinear instability of Euler and Prandtl equations, Comm. Pure Appl. Math., 53(2000), 1067-1091.

[11] A. Kiselev and V. Sverák, Small scale creation for solutions of the incompressible two-dimensional Euler equation, Ann. Math., 180(2014), 1205-1220.

[12] L. Landau, On the vibration of the electronic plasma, J. Phys. USSR, 10(1946), 25.

[13] Z. Lin, Instabilty of some ideal plane flows, SIAM J. Math. Anal., 35(2003), 318-356.

[14] Z. Lin, Nonlinear instability of ideal plane flows, Int. Math. Res. Not., 41(2004), 2147-2178.

[15] Z. Lin and C. Zeng, Small BGK waves and nonlinear Landau damping, Comm. Math. Phys., 306(2011), 291-331.

[16] Z. Lin and C. Zeng, Inviscid dynamic structures near Couette flow, Arch. Rat. Mech. Anal., 200(2011), 1075-1097.

[17] A. J. Majda and A. L. Bertozzi, Vorticity and Incompressible Flow, Cambridge Texts Appl. Math. 27, Cambridge University Press, Cambridge, 2002.

[18] C. Mouhot and C. Villani, On Landau damping, Acta Math., 207(2011), 29-201.

[19] W. Orr, Stability and instability of steady motions of a perfect liquid, Proc. Ir. Acad. Sect. A: Math Astron. Phys. Sci., 27(1907), 9-66.

[20] L. Rayleigh, On the stability or instability of certain fluid motions, Proc. London Math. Soc., 9(1880), 57-70.

[21] S. I. Rosencrans and D. H. Sattinger, On the spectrum of an operator occurring in the theory of Hydrodynamics stability, J. Math. Phys., 45(1966), 289-300.

[22] P. J. Schmid and D. S. Henningson, Stability and transition in shear flows, Applied Mathematical Sciences Vol. 142, Springer-Verlag, New York, 2001.

[23] S. A. Stepin, Nonself-adjoint Friedrichs models in Hydrodynamics stability, Functional Analysis and its Applications, 29(1995), 91-101.

[24] M. M. Vishik and S. Friedlander, Nonlinear instability in two dimensional ideal fluids: the case of a dominant eigenvalue, Comm. Math. Phys., 243(2003), 261-273.

[25] C. Zillinger, Linear inviscid damping for monotone shear flows, arXiv:1410.7341.

[26] C. Zillinger, Linear inviscid damping for monotone shear flows in a finite periodic channel, boundary effects, blow-up and critical Sobolev regularity, arXiv:1506.04010. 
School of Mathematical Science, Peking University, 100871, Beijing, P. R. China E-mail address: jnwdyi@163.com

School of Mathematical Science, Peking University, 100871, Beijing, P. R. China E-mail address: zfzhang@math.pku.edu.cn

Department of Mathematics, Zhejiang University, 310027, Hangzhou, P. R. China E-mail address: zjzjzwr@126.com 